UNIVERSIDADE DE BRASÍLIA

FACULDADE DE TECNOLOGIA

DEPARTAMENTO DE ENGENHARIA ELÉTRICA

IDENTIFICAÇÃO DE MODOS DE OSCILAÇÃO UTILIZANDO FILTRO DE KALMAN ESTENDIDO

PEDRO ELIAS WEBER DE DEUS AMARAL

ORIENTADOR: FRANCISCO DAMASCENO FREITAS

DISSERTAÇÃO DE MESTRADO EM ENGENHARIA ELÉTRICA

PUBLICAÇÃO: PPGEE.DM - 613/2015

BRASÍLIA/DF: DEZEMBRO - 2015. 
UNIVERSIDADE DE BRASÍLIA

FACULDADE DE TECNOLOGIA

DEPARTAMENTO DE ENGENHARIA ELÉTRICA

\section{IDENTIFICAÇÃO DE MODOS DE OSCILAÇÃO UTILIZANDO FILTRO DE KALMAM ESTENDIDO}

\section{PEDRO ELIAS WEBER DE DEUS AMARAL}

DISSERTAÇÃO DE MESTRADO SUBMETIDA AO DEPARTAMENTO DE ENGENHARIA ELÉTRICA DA FACULDADE DE TECNOLOGIA DA UNIVERSIDADE DE BRASÍLIA, COMO PARTE DOS REQUISITOS NECESSÁRIOS PARA A OBTENÇÃO DO GRAU DE MESTRE.

APROVADA POR:

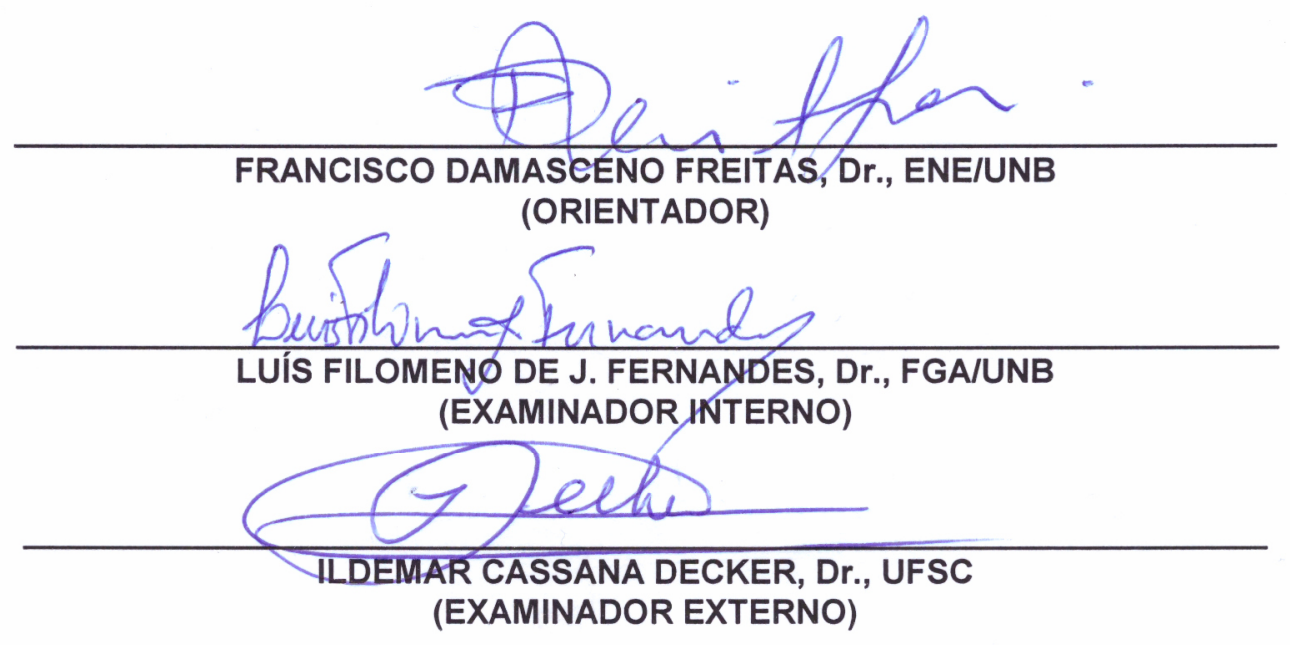

Brasília, 15 de dezembro de 2015. 


\section{FICHA CATALOGRÁFICA}

AMARAL, PEDRO ELIAS WEBER DE DEUS

Identificação de Modos de Oscilação Utilizando Filtro de Kalman Estendido.

[Distrito Federal] 2015.

xviii, 117p., 210 x 297 mm (ENE/FT/UnB, Mestre, Engenharia Elétrica, 2015).

Dissertação de Mestrado - Universidade de Brasília.

Faculdade de Tecnologia.

Departamento de Engenharia Elétrica.

1. Modos de oscilação eletromecânica

2. PMU

3. Filtro de Kalman Estendido

4. Dados de Ambiente

I. $\mathrm{ENE} / \mathrm{FT} / \mathrm{UnB}$

II. Título (série)

\section{REFERÊNCIA BIBLIOGRÁFICA}

AMARAL, P. E. W. D. (2015). Identificação de Modos de Oscilação Utilizando Filtro de Kalman Estendido. Dissertação de Mestrado em Engenharia Elétrica, Publicação PPGEE.DM - 613/15, Departamento de Engenharia Elétrica, Faculdade de Tecnologia, Universidade de Brasília, Brasília, DF, 117p.

\section{CESSÃO DE DIREITOS}

NOME DO AUTOR: Pedro Elias Weber de Deus Amaral.

TÍTULO DA DISSERTAÇÃO DE MESTRADO: Identificação de Modos de Oscilação Utilizando Filtro de Kalman Estendido.

GRAU / ANO: $\quad$ Mestre / 2015

É concedida à Universidade de Brasília permissão para reproduzir cópias desta dissertação de mestrado e para emprestar ou vender tais cópias somente para propósitos acadêmicos e científicos. O autor reserva outros direitos de publicação e nenhuma parte desta dissertação de mestrado pode ser reproduzida sem a autorização por escrito do autor.

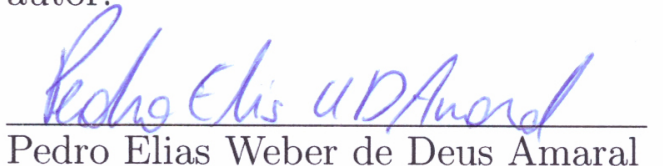

Área Especial 4, Módulos E/F, Bloco A, Apto. 1504, Guará II 71.070-654 Brasília - DF - Brasil. 


\section{DEDICATÓRIA}

Este trabalho é dedicado

à minha esposa Patrícia,

ao meu filho João Pedro,

aos meus pais Izabel e Nelson

e aos meus irmãos Lívia e Gustavo. 


\section{AGRADECIMENTOS}

À minha esposa Patrícia pelo amor, companheirismo, dedicação, compreensão, paciência, garra para cuidar do nosso príncipe em minhas ausências, por entender minhas ausências diárias, pelo apoio e carinho essenciais à minha tranquilidade para mais essa vitória.

Ao meu príncipe João Pedro por receber o papai todos os dias com um sorriso lindo e reconfortante, por dar sentido a vida do papai e da mamãe, e por compreender, ainda que com poucos meses de vida, que minhas ausências diárias tinham o único propósito de trazer conforto e aprendizado à nossa família.

Aos meus pais Izabel e Nelson pelo amor, educação e suporte fundamentais em toda minha existência.

Aos meus irmãos Lívia e Gustavo pelo amor e por cuidarem tão bem de nossos pais desde que me mudei de Goiânia.

Ao Professor Damasceno pelo aprendizado, pela paciência e brilhante orientação.

Aos colegas da Agência Nacional de Energia Elétrica pela convivência, incentivo e oportunidade de me dedicar à conclusão deste trabalho.

Aos Colegas e Professores da Escola de Engenharia Elétrica da Universidade Federal de Goiás pelo aprendizado dos anos de graduação.

A todos que compõe o Departamento de Engenharia Elétrica da Universidade de Brasília pela oportunidade de realização deste trabalho e pela acolhida.

A todos os amigos e familiares que de alguma forma me ajudaram a ser e chegar onde estou.

E a Deus, pela vida, pela saúde da minha família e pela oportunidade de conviver com todas as pessoas citadas acima que fazem parte de minha vida! 


\title{
RESUMO
}

\section{IDENTIFICAÇÃO DE MODOS DE OSCILAÇÃO UTILIZANDO FILTRO DE KALMAN ESTENTIDO}

\author{
Autor: Pedro Elias Weber de Deus Amaral \\ Orientador: Francisco Damasceno Freitas \\ Programa de Pós-graduação em Engenharia Elétrica \\ Brasília, dezembro de 2015
}

A estabilidade de um sistema elétrico de potência é um dos requisitos para que as cargas do sistema sejam atendidas com qualidade e sem interrupções. O adequado monitoramento e o amortecimento dos modos de oscilação eletromecânica são fundamentais para o controle da estabilidade de um sistema elétrico.

Esta dissertação apresenta um método para identificação de modos de oscilação eletromecânica cujo fundamento é baseado no princípio do Filtro de Kalman Estendido. Os modos são calculados a partir de parâmetros que também são estados do filtro. A metodologia é voltada para identificação de parâmetros característicos de sinais obtidos de PMU (Phasor Measurement Units). Todos os sinais obtidos e utilizados para testes são de baixa amplitude, conhecidos como dados de ambiente.

A técnica proposta foi avaliada por meio de dois tipos de dados. Em um primeiro tipo, os sinais foram simulados. Com esta finalidade, utilizou-se o software Power System Toolbox (PST). Em outros testes, foram considerados dados de campo, obtidos de PMUs instaladas em baixa tensão localizados em universidades brasileiras em diversos estados. Considerando a natureza estocástica dos sinais, o método proposto identificou adequadamente os modos dominantes presentes nos dados de ambiente simulados do sistema teste, tanto em situações em que a frequência e amortecimento do modo eram fixos, quanto em situações em que os parâmetros do modo variavam ao longo do período de simulação. No caso dos resultados determinados para os dados de campo, foi identificada a presença de modo de oscilação dominante, na faixa entre $0,2 \mathrm{~Hz}$ e 0,4 Hz. Este é um modo interárea, característico do Sistema Interligado Nacional (SIN). Portanto, em ambos os testes a metodologia proposta mostrou-se adequada para identificação de modos dominantes a partir de dados de ambiente. 


\title{
ABSTRACT \\ ELECTROMECHANICAL MODE IDENTIFICATION USING EXTEN- DED KALMAN FILTER
}

\author{
Author: Pedro Elias Weber de Deus Amaral \\ Supervisor: Francisco Damasceno Freitas \\ Programa de Pós-graduação em Engenharia Elétrica \\ Brasília, December of 2015
}

Power Systems must be stable for the system loads to be supplied continiously and with good quality. The proper monitoring and damping of electromechanical oscillation modes are fundamental for the power system stability control.

This thesis presents a method for identification of electromechanical oscillation modes based on the Extended Kalman Filter. The modes are calculated from parameters which are also state variables of the filter. The methodology aims to identify characteristic parameters of signals obtained from PMU (Phasor Measurement Units). All the signals obtained and used in tests are low amplitude signals, also known as ambient data.

The proposed methodology was evaluated by two kinds of data. In a first kind, the signals were simulated. For this purpose, it was used the Power System Toolbox software (PST). In the second kind, it was considered field data obtained from PMUs installed at low voltage in Brazilian universities in several states. Considering the stochastic nature of the data, the proposed method identified the dominant modes present in the simulated ambient data of the test system accordingly, both in situations in which the frequency and damping of the mode were fixed, as in situations in which the mode parameters varied throughout the period of simulation. In the case of the field data, the results identified the presence of dominant oscillation mode in the frequency range between $0,2 \mathrm{~Hz}$ and $0,4 \mathrm{~Hz}$. This is an inter-area mode, characteristic of the Brazilian National Grid. Therefore, in both kinds of data, the proposed methodology was appropriate to identify dominant modes from ambient data. 


\section{SUMÁRIO}

1 Introdução 1

1.1 Apresentação . . . . . . . . . . . . . . . . . . 1

1.2 Motivação . . . . . . . . . . . . . . . . . . . . . . . . . 2

1.3 Objetivo . . . . . . . . . . . . . . . . . . 3

1.4 Publicação . . . . . . . . . . . . . . . . . . . 4

1.5 Organização da Dissertação . . . . . . . . . . . . . . . . 4

2 Estabilidade de Sistemas Elétricos de Potência $\quad 6$

2.1 Introdução . . . . . . . . . . . . . . . . . . . . . . . . . . . 6

2.2 Classificação da estabilidade de SEPs . . . . . . . . . . . . . . . 6

2.3 Modelo Linearizado de SEPs . . . . . . . . . . . . . . . . . . . . . . 10

2.4 Avaliação da estabilidade de SEPs utilizando medições fasoriais sincronizadas . . . . . . . . . . . . . . . . . . . . . . 12

2.5 Revisão Bibliográfica . . . . . . . . . . . . . . . . . . 14

2.6 Conclusão . . . . . . . . . . . . . . . . . . . . . . . . 17

3 Medições, Métodos e Modelagem $r$

3.1 Introdução . . . . . . . . . . . . . . . . . . . . . . . . . . . . . 18

3.2 Unidades de Medição Fasorial Sincronizada . . . . . . . . . . . . . . . . 18

3.3 Pré-processamento de sinais . . . . . . . . . . . . . . . . . . . . . . 19

3.3.1 Interpolação de sinais de PMUs . . . . . . . . . . . . . 20

3.3.2 Ajuste dos valores de diferença angular . . . . . . . . . . . . . 29

3.3.3 Filtragem dos sinais . . . . . . . . . . . . . . . . . 31

3.4 Filtro de Kalman . . . . . . . . . . . . . . . . . . . . . . . . . . . . 32

3.4.1 Algoritmo do Filtro de Kalman . . . . . . . . . . . . . . . . . . 44

3.4.2 Algoritmo do Filtro de Kalman Estendido . . . . . . . . . . . . 45

3.4.3 Identificação de parâmetros na forma de estados . . . . . . . . . 46

3.5 Modelagem do Sistema . . . . . . . . . . . . . . . . . . . . 47

3.6 Ajustes do Filtro de Kalman . . . . . . . . . . . . . . . . . . . . . . . . 52

3.6.1 Ajuste das Covariâncias $Q$ e $R \ldots \ldots$. . . . . . . . 53 
3.6.2 Ajuste do valor inicial do vetor de estados, $\hat{x}_{0}$, e da matriz de covariâncias do erro de estimação de estados, $P_{0} \ldots \ldots$. . . . 54

3.6.3 Ajuste do número de modos de oscilação a serem estimados . 56

3.7 Conclusão . . . . . . . . . . . . . . . . . . . . . . . . . . . 59

4 Testes e Resultados $\quad 60$

4.1 Introdução . . . . . . . . . . . . . . . . . . . . . . 60

4.2 Simulações . . . . . . . . . . . . . . . . . . . . 60

4.2.1 Sistema teste para simulações . . . . . . . . . . . . . . 60

4.2 .2 Primeiro caso - um modo dominante fixo . . . . . . . . . . . . 62

4.2.3 Segundo caso - um modo dominante fixo . . . . . . . . . . . . 63

4.2.4 Terceiro caso - um modo dominante variável . . . . . . . . . . . 66

4.2.5 Quarto caso - um modo dominante fixo . . . . . . . . . . . 67

4.2.6 Quinto caso - um modo dominante fixo . . . . . . . . . . . 70

4.3 Aplicações em sinais de PMUs do SIN . . . . . . . . . . . . . . . 74

4.3.1 Sinais com referência na UFC . . . . . . . . . . . . . . 77

4.3.2 Sinais com referência na UFT . . . . . . . . . . . . . . 81

4.3 .3 Sinais com referência na UFMA . . . . . . . . . . . . . 85

4.4 Conclusão . . . . . . . . . . . . . . . . . . . . . . . . . . . . . . 87

5 Conclusão $\quad 89$

5.1 Conclusões gerais . . . . . . . . . . . . . . . . . . . . 89

5.2 Sugestões para trabalhos futuros . . . . . . . . . . . . . . . . 90

REFERÊNCIAS BIBLIOGRÁFICAS $\quad 92$ 


\section{LISTA DE TABELAS}

2.1 Principais modos de oscilação interárea do SIN. . . . . . . . . . . . . 9

4.1 Modo de oscilação constante do primeiro caso. . . . . . . . . . . . . . . 62

4.2 Modo de oscilação constante do segundo caso. . . . . . . . . . . . . . . 64

4.3 Modo de oscilação variável do terceiro caso. . . . . . . . . . . . . . . 66

4.4 Modo de oscilação constante do quarto caso. . . . . . . . . . . . . . . 67

4.5 Modo de oscilação constante do quinto caso . . . . . . . . . . . . . . . . 70

4.6 Faixas de valores de frequência de modos de oscilação interárea conhecidos do SIN . . . . . . . . . . . . . . . . . . . . . . . . 76

4.7 Modo de oscilação identificado nos sinais com referência na PMU da UFC 80

4.8 Modo de oscilação identificado nos sinais com referência na PMU da UFT 84 


\section{LISTA DE FIGURAS}

2.1 Classificação da estabilidade de SEP. . . . . . . . . . . . . . . . . . . . 7

2.2 Fluxo de Potência na Linha de Transmissão Malin-Round Mountain 1 durante o blackout ocorrido no WECC em 1996. . . . . . . . . . . . . . 10

2.3 Filtro branqueador. . . . . . . . . . . . . . . . . . . . . . 15

3.1 Arquitetura de um sistema de medição fasorial sincronizada. . . . . . . 19

3.2 PMUs instaladas no âmbito do Projeto MedFasee. . . . . . . . . . . . . 19

3.3 Ângulo da fase A do sinal de tensão da UnB . . . . . . . . . . . . . . . 21

3.4 Diferença angular de tensão da fase A entre as PMUs instaladas na UnB e na UFAC . . . . . . . . . . . . . . . . . . . 22

3.5 Ângulo da fase A do sinal de tensão da UnB sem interpolação e com interpolação linear . . . . . . . . . . . . . . . . . . . . . .

3.6 Diferença angular de tensão da fase A entre as PMUs instaladas na UNB e na UFAC utilizando interpolação linear . . . . . . . . . . . . . . .

3.7 Ângulo de tensão da fase A das PMUs instaladas na UnB, com interpolação linear, e na UFAC, sem interpolação. . . . . . . . . . . . . . . .

3.8 Ângulo de tensão da fase A da PMU instalada na UnB com interpolação linear e interpolação PCHIP . . . . . . . . . . . . . . . .

3.9 Diferença angular de tensão da fase A entre as PMUs instaladas na UnB e na UFAC utilizando interpolação linear e PCHIP . . . . . . . . . . .

3.10 Diferença angular de tensão da fase A entre as PMUs instaladas na UnB e na UFAC, utilizando interpolação linear, antes e após a filtragem passa-alta . . . . . . . . . . . . . . .

3.11 Diferença angular de tensão da fase A entre as PMUs instaladas na UnB e na UFAC, utilizando interpolação PCHIP, antes e após a filtragem passa-alta. . . . . . . . . . . . . . . . . .

3.12 Ângulo de tensão da fase A da PMU instalada na UFRGS sem interpolação, com interpolação linear e com interpolação PCHIP . . . . . . .

3.13 Ângulo de tensão da fase A das PMUs instaladas na UFRGS e na UnB com interpolação linear e PCHIP. . . . . . . . . . . . . . . . . 29

3.14 Ângulo de tensão da fase A das PMUs instaladas na UnB e na UFSC . 30 
3.15 Diferença angular de tensão da fase A entre as PMUs instaladas na UnB e na UFSC antes do ajuste de valores angulares . . . . . . . . . . . .

3.16 Diferença angular de tensão da fase A entre as PMUs instaladas na UnB e na UFSC após ajuste de valores angulares . . . . . . . . . . . . . .

3.17 Espectro de frequências do sinal de diferença angular de tensão antes da filtragem . . . . . . . . . . . . . . . . . . .

3.18 Espectro de frequências do sinal de diferença angular de tensão após a filtragem . . . . . . . . . . . . . . . . .

3.19 Espectro de frequências do sinal de diferença angular de tensão antes e após a filtragem . . . . . . . . . . . . . . . . . . . 33

3.20 Densidade de probabilidade da localização baseada na estimativa $z_{1} \ldots \quad$. 35

3.21 Densidade de probabilidade da localização baseada na estimativa $z_{2}$. . 36

3.22 Densidade de probabilidade da localização baseada nas estimativas $z_{1} \mathrm{e}$

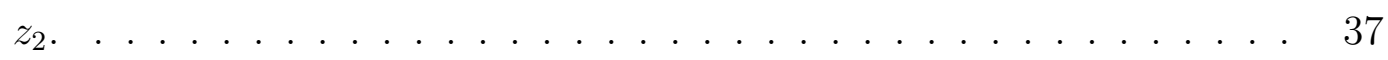

3.23 Diagrama de blocos de um modelo de estimador de estados. . . . . . . 39

3.24 Aplicação do FKE para $Q=R=0,01 \ldots \ldots$. . . . . . . 54

3.25 Aplicação do FKE para $Q=R=1 \ldots \ldots \ldots$. . . . . . . 54

3.26 Aplicação do FKE para $Q=R=100 \ldots \ldots$. . . . . . . . . . 55

3.27 Aplicação do FKE para vários valores de $\hat{x}_{0}$ e de $P_{0} \ldots \ldots$. . . . . 56

3.28 Espectro de frequências de um sinal de dados de ambiente . . . . . . . 58

3.29 Espectro de frequências de um sinal de dados de ambiente e as frequências de modos hipotéticos identificados: a) um modo de oscilação identificado; b) dois modos de oscilação identificados; c) três modos de oscilação identificados . . . . . . . . . . . . . . . . . . 5 58

4.1 Diagrama unifilar do sistema teste no PST . . . . . . . . . . . 61

4.2 Frequência e amortecimento do modo de oscilação para o primeiro caso 63

4.3 Espectro de frequências do sinal simulado no primeiro caso com destaque para a frequência do modo de oscilação identificado . . . . . . . . . . . .

4.4 Frequência e amortecimento do modo de oscilação identificado no segundo caso . . . . . . . . . . . . . . . . . . . .

4.5 Espectro de frequências do sinal simulado no segundo caso com destaque para a frequência do modo de oscilação identificado . . . . . . . . . . .

4.6 Frequência e amortecimento do modo de oscilação variável identificado no terceiro caso . . . . . . . . . . . . . . . . . . . . . . .

4.7 Frequência e amortecimento do modo de oscilação identificado no quarto caso utilizando modelagem com um modo de oscilação . . . . . . . . . 
4.8 Espectro de frequências do sinal simulado no quarto caso utilizando um modelo com um modo com destaque para a frequência do modo de oscilação identificado . . . . . . . . . . . . . . . . . . . .

4.9 Espectro de frequências do sinal simulado no quarto caso utilizando um modelo com dois modos com destaque para as frequências dos dois modos de oscilação identificados . . . . . . . . . . . . . . . . . . .

4.10 Frequência e amortecimento do modo de oscilação identificado no quarto caso utilizando modelagem com dois modos de oscilação . . . . . . . . . .

4.11 Frequência e amortecimento do modo de oscilação identificado no quinto caso utilizando modelagem com um modo de oscilação . . . . . . . . .

4.12 Espectro de frequências do sinal simulado no quinto caso utilizando um modelo com um modo com destaque para a frequência do modo de oscilação identificado . . . . . . . . . . . . . . . . . . .

4.13 Espectro de frequências do sinal simulado no quinto caso utilizando um modelo com dois modos com destaque para a frequência dos dois modos de oscilação identificados . . . . . . . . . . . . . . . . . . . .

4.14 Frequência e amortecimento do modo de oscilação identificado no quinto caso utilizando modelagem com dois modos de oscilação . . . . . . . . . .

4.15 Espectro de frequências do sinal simulado no quinto caso utilizando um modelo com três modos com destaque para a frequência dos três modos de oscilação identificados . . . . . . . . . . . . . . . . . .

4.16 Frequência e amortecimento de um dos modos de oscilação identificados no quinto caso utilizando modelagem com três modos de oscilação . . .

4.17 Frequência e amortecimento de um dos modos de oscilação identificados no quinto caso utilizando modelagem com três modos de oscilação . . .

4.18 Espectro de frequências dos sinais: a) defasagem angular de tensão entre a PMU da UFC e a da UFRGS; b) entre a PMU da UFC e a da UFSC; c) entre a PMU da UFC e a da UNIPAMPA e d) entre a PMU da UFC e a da UTFPR.

4.19 Espectro de frequências, com destaque em vermelho para as frequências dos dois modos identificados, dos sinais: a) defasagem angular de tensão entre a PMU da UFC e a da UFRGS; b) entre a PMU da UFC e a da UFSC; c) entre a PMU da UFC e a da UNIPAMPA e d) entre a PMU da UFC e a da UTFPR. . . . . . . . . . . . . . . . . . . . 
4.20 Espectro de frequências, com destaque em vermelho para as frequências dos três modos identificados, dos sinais: a) defasagem angular de tensão entre a PMU da UFC e a da UFRGS; b) entre a PMU da UFC e a da UFSC; c) entre a PMU da UFC e a da UNIPAMPA e d) entre a PMU da UFC e a da UTFPR. . . . . . . . . . . . . . . . . . . . . . . .

4.21 Amortecimento e frequência do modo de oscilação identificado nos sinais de defasagem angular de tensão entre as PMUs da UFRGS, UFSC, UNIPAMPA e UTFPR em relação à da UFC, utilizando modelagem com dois modos . . . . . . . . . . . . . . . . . . . .

4.22 Média móvel de 10 minutos do amortecimento e da frequência do modo de oscilação identificado nos sinais de defasagem angular de tensão . . .

4.23 Comparação dos valores de amortecimento e frequência do modo de oscilação identificado utilizando modelagem com dois e três modos . . .

4.24 Espectro de frequências dos sinais: a) defasagem angular de tensão entre a PMU da UFT e a da UFRGS; b) entre a PMU da UFT e a da UFSC; c) entre a PMU da UFT e a da UNIPAMPA e d) entre a PMU da UFT e a da UTFPR.

4.25 Espectro de frequências, com destaque em vermelho para as frequências dos dois modos identificados, dos sinais: a) defasagem angular de tensão entre a PMU da UFT e a da UFRGS; b) entre a PMU da UFT e a da UFSC; c) entre a PMU da UFT e a da UNIPAMPA e d) entre a PMU da UFT e a da UTFPR. . . . . . . . . . . . . . . . . . . . .

4.26 Espectro de frequências, com destaque em vermelho para as frequências dos três modos identificados, dos sinais: a) defasagem angular de tensão entre a PMU da UFT e a da UFRGS; b) entre a PMU da UFT e a da UFSC; c) entre a PMU da UFT e a da UNIPAMPA e d) entre a PMU da UFT e a da UTFPR. . . . . . . . . . . . . . . . . . . .

4.27 Amortecimento e frequência do modo de oscilação identificado nos sinais de defasagem angular de tensão entre as PMUs da UFRGS, UFSC, UNIPAMPA e UTFPR em relação à da UFT, utilizando modelagem com três modos . . . . . . . . . . . . . . . . . . . . . . . .

4.28 Média móvel de 10 minutos do amortecimento e da frequência do modo de oscilação identificado nos sinais de defasagem angular de tensão . . .

4.29 Comparação dos valores de amortecimento e frequência do modo de oscilação identificado utilizando modelagem com dois e três modos . . .

4.30 Espectro de frequências do sinal de defasagem angular de tensão entre a PMU da UFC e a da UFMA. 
4.31 Amortecimento e frequência dos três polos calculados para o sinal de defasagem angular de tensão entre a PMU da UFC e a da UFMA, utilizando modelagem com três modos . . . . . . . . . . . . . . 87 


\section{LISTA DE SÍMBOLOS}

\section{Siglas}

$\mathrm{AR}$

Autoregressive (Autorregressivo).

ARMA Autoregressive Moving Average (Autorregressivo de Média Móvel).

ARMAX Autoregressive Moving Average with Exogenous Inputs (Autorregressivo de Média Móvel com Entradas Exógenas).

FACTS Flexible AC Transmission System (Sistema de Transmissão AC Flexível).

FFT Fast Fourier Transform (Transformada Rápida de Fourier).

FK Filtro de Kalman.

FKE Filtro de Kalman Estendido.

GPS Global Positioning System (Sistema de Posicionamento Global).

LMS Least Mean Squares.

MOESP Multivariable Output Error State Space Algorithm.

N4SID Numerical Algorithms for Subspace State Space System Identification.

ONS Operador Nacional do Sistema Elétrico

PCHIP Piecewise Cubic Hermite Interpolating Polynomial.

PDC Phasor Data Concentrator (Concentrador de Dados Fasoriais).

PMU Phasor Measurement Unit (Unidade de Medição Fasorial).

PSS Power System Stabilizers (Estabilizador de Sistema de Potência).

PST Power System Toolbox.

RLS Recursive Least Squares.

RRLS Robust Recursive Least Squares.

R3LS Regularized Robust Recursive Least Squares.

SCADA Supervisory Control and Data Acquisition.

SEP Sistema Elétrico de Potência.

SIN Sistema Interligado Nacional.

SMFS Sistema de Medição Fasorial Sincronizada.

UFAC Universidade Federal do Acre.

UFC Universidade Federal do Ceará.

UFMA Universidade Federal do Maranhão.

UFRGS Universidade Federal do Rio Grande do Sul.

UFSC Universidade Federal de Santa Catarina.

UFT Universidade Federal do Tocantins.

UnB Universidade de Brasília. 
UNIPAMPA Universidade Federal do Pampa.

UTFPR Universidade Tecnológica Federal do Paraná.

WECC Western Electricity Coordinating Council.

\section{Símbolos Latinos}

\begin{tabular}{|c|c|}
\hline$a_{k}$ e $b_{k}$ & Parâmetros que definem os modos de oscilação. \\
\hline$e$ & Erro. \\
\hline$h$ & Período de amostragem, em segundos. \\
\hline$I_{n}$ & Matriz identidade de ordem $n$. \\
\hline$j$ & Unidade imaginária $(\sqrt{-1})$ de um número complexo. \\
\hline$k$ & Numero inteiro representativo de tempo discreto. \\
\hline$K$ & Matriz de ganho de Kalman. \\
\hline$P$ & Matriz de covariâncias das estimativas a posteriori. \\
\hline$P^{-}$ & Matriz de covariâncias das estimativas a priori. \\
\hline $\bar{Q}$ & Matriz diagonal de covariâncias de ruído de modelo. \\
\hline$\overline{Q_{P}}$ & $\begin{array}{l}\text { Matriz de covariâncias de ruído de modelo associada aos } \\
\text { parâmetros dos modos de oscilação. }\end{array}$ \\
\hline$\overline{Q_{X}}$ & Matriz de covariâncias de ruído de modelo associada aos estados. \\
\hline$Q$ & Elemento da diagonal da matriz de covariâncias de ruído de modelo. \\
\hline$Q_{P}$ & $\begin{array}{l}\text { Covariância de ruído de modelo associada aos parâmetros dos mo- } \\
\text { dos de oscilação. }\end{array}$ \\
\hline$Q_{P a}$ & Covariância de ruído de modelo associada aos parâmetros $a_{k}$. \\
\hline$Q_{P b}$ & Covariância de ruído de modelo associada aos parâmetros $b_{k}$. \\
\hline$Q_{X}$ & Covariância de ruído de modelo associada aos estados. \\
\hline $\bar{R}$ & Matriz de covariâncias de ruído de medição. \\
\hline$R$ & $\begin{array}{l}\text { Elemento da diagonal da matriz de covariâncias de ruído de } \\
\text { medição. }\end{array}$ \\
\hline $\mathbb{R}$ & Conjunto dos números reais. \\
\hline$t$ & Tempo. \\
\hline$x$ & Vetor de estados. \\
\hline$\hat{x}$ & Vetor de estados estimados a posteriori. \\
\hline$\hat{x}^{-}$ & Vetor de estados estimados a priori. \\
\hline$w_{k}$ & Ruído de modelo associado aos parâmetros dos modos de oscilação. \\
\hline$w_{p}$ & Ruído de modelo associado aos estados. \\
\hline$y$ & Vetor de medições. \\
\hline$\hat{y}$ & Vetor de medições estimadas. \\
\hline
\end{tabular}


$v \quad$ Ruído de medição.

z Dados de medição.

H Matriz cujos parâmetros são conhecidos.

J Função de custo.

\section{Símbolos Gregos}

$\theta$

$\mu$

$\xi$

$\sigma$

$\sigma_{z}$

$\sigma_{z}^{2}$

$\omega$

Vetor de parâmetros a serem estimados pelo Filtro de Kalman Estendido.

Autovalor de uma matriz.

Média de uma conjunto de medições.

Taxa de amortecimento de um modo de oscilação eletromecânica.

Parte real de um autovalor.

Desvio padrão da medida $z$.

Variância da medida $z$.

Parte imaginária de um autovalor. 


\section{Introdução}

\subsection{Apresentação}

O Sistema Interligado Nacional (SIN) é composto por inúmeras linhas de transmissão de energia elétrica, cargas industriais, comerciais e residenciais, e diversas centrais de geração localizadas em todo o território nacional.

O adequado funcionamento do SIN, bem como de outros sistemas elétricos de potência (SEP), pressupõe o atendimento contínuo das cargas com qualidade, sem interrupções. Um dos requisitos desejáveis de um SEP é que ele opere continuamente e de forma estável.

Historicamente, o maior problema em termos de estabilidade de SEP tem sido caracterizado por instabilidades transitórias, principalmente aquelas causadas por grandes distúrbios no sistema. Com a evolução natural dos sistemas, o aumento do número de interconexões de grandes áreas produtoras e consumidoras de energia elétrica, aumento da complexidade dos sistemas de controle, inserção intensa de fontes de energia renováveis e a operação dos sistemas com carregamento elevado, surgiram dificuldades adicionais de controle dos SEPs. Desta forma, ferramentas de controle e monitoramento visando preservar a estabilidade do sistema são sempre benéficas $[1,2]$.

Os SEPs estão sujeitos a oscilações eletromecânicas locais e remotas provocadas pelas interações elétricas entre as unidades de geração [3]. As oscilações bem amortecidas, em geral, são características dos sistemas elétricos na maior parte do tempo de operação [4]. No entanto, no curto período em que são pouco amortecidas, ou mesmo instáveis, as oscilações eletromecânicas deterioram a segurança da operação do sistema e podem levar a restrições de fluxo de potência em linhas de transmissão e até ao colapso do sistema [5].

O estudo de oscilações eletromecânicas usualmente é feito no âmbito dos estudo de estabilidade a pequenas perturbações utilizando técnicas de análise de sistemas lineares $[2,6]$. Recentemente, dados obtidos de unidades de medição fasorial (Phasor Measurement Unit - PMU) têm sido utilizados de forma complementar para estimação de 
modos de oscilação eletromecânica em SEPs em tempo real [5, 7, 8, 9]. O advento de novas tecnologias de processadores, a transmissão de dados via Internet e o uso de dispositivos que permitem a sincronização temporal de dados, como o Global Positioning System (GPS), têm tornado viável a medição de grandezas em locais bastante remotos dos locais de controle. Um exemplo de projeto bem sucedido desta natureza é o identificado em [10], denominado Medfasee.

Os sinais medidos pelas PMUs permitem que algumas grandezas características do sistema possam ser estimadas. Algumas delas são ângulo de tensão e frequência. Estas grandezas podem ser utilizadas para a avaliação de características dinâmicas do sistema, mesmo em tempo real. Muitos trabalhos destacam o cálculo de modos de oscilação eletromecânica dominantes como aplicação que faz uso desses sinais.

Sabe-se que em grande parte do tempo, o sistema opera sob excitação causada principalmente pelo chaveamento permanente de cargas, chamada de ruído ambiente [11]. Neste sentido, utilizar dados de ambiente para estimação em tempo real das características dinâmicas do sistema torna-se uma importante contribuição para o setor elétrico.

\subsection{Motivação}

A estabilidade de um SEP a pequenas perturbações usualmente é avaliada a partir do modelo linearizado do sistema para um dado ponto de operação utilizando técnicas de análise de sistemas lineares $[2,6]$. Se o modelo linearizado for estável, isso implica que o ponto de operação é estável [6]. No entanto, o sistema não linear poderá ser instável para uma grande perturbação.

Modelos lineares de SEPs são formados por equações de espaço de estados de ordem elevada, podendo ter centenas de milhares de estados. Por esse motivo, geralmente são aplicadas técnicas diversas de análise de sistemas lineares esparsos e redução da ordem de modelos, preservando as principais características dinâmicas do sistema nas faixas de frequência de interesse $[2,12,13,14,15,16,17,18,19]$.

Os modelos matemáticos utilizados são complexos e utilizam parâmetros que podem variar no tempo. Em vista disso, dados obtidos de PMUs têm sido utilizados de forma complementar para estimação de modos de oscilação eletromecânica em sistemas elétricos, possibilitando a análise quase em tempo real da estabilidade dos SEP a 
pequenas perturbações $[2,5,7,8,9,20,21,22,23]$.

Muitos trabalhos [3, 22, 24, 25, 26, 27, 28, 29] investigam os sinais transitórios, chamados de ringdown, originados a partir de grandes perturbações, como curtos-circuitos, perda de algum equipamento, etc. No entanto, o sistema opera a maior parte do tempo apenas sob excitação de ruído ambiente, caracterizado pela variação estocástica de cargas que excitam os modos de oscilação presentes [11].

Deste modo, utilizar dados de ambiente ao invés de provenientes de ringdown, para estimação em tempo real das características dinâmicas de SEPs, torna-se uma importante contribuição para o setor elétrico, abrindo a possibilidade para o monitoramento em tempo quase real dos modos de oscilação dominantes no sistema. A habilidade de controlar as oscilações interárea permite ao operador melhorar a confiabilidade e otimizar a capacidade de transmissão de energia elétrica do sistema, minimizando possíveis interrupções de carga [2].

\subsection{Objetivo}

O objetivo deste trabalho é propor uma técnica para estimação de modos de oscilação eletromecânica dominantes de sistemas elétricos a partir de dados de ambiente provenientes de PMUs. A técnica para estimação de parâmetros utiliza abordagem baseada no Filtro de Kalman Estendido (FKE), permitindo a observação da variação dos parâmetros do modo dominante no tempo com modelos de ordem reduzida.

Os objetivos específicos deste trabalho são os seguintes:

- Utilização do FKE para a estimação de parâmetros desconhecidos na forma de sistemas de espaço de estados;

- Simulação de medições por PMUs de dados de ambiente utilizando o software livre de análise de SEP Power System Toolbox (PST);

- Pré-processamento de dados de PMUs instaladas no SIN para aplicação da técnica baseada no FKE proposta neste trabalho.

A identificação de parâmetros desconhecidos em modelos de sistemas lineares na forma de sistemas de espaço de estados foi realizada utilizando o FKE. Para tanto, os parâmetros desconhecidos foram tratados como variáveis de estado a serem identificadas, tornando o modelo não linear. 
Os testes com a metodologia proposta foram feitos inicialmente a partir de dados simulados no software PST. Nas simulações realizadas, as cargas do sistema teste foram alteradas de forma que $1 \%$ da carga variasse aleatoriamente. As medições das grandezas do sistema foram feitas a uma taxa de 60 amostras por segundo, de modo que foi possível simular medições feitas por PMUs de dados de ambiente no sistema teste.

O método proposto foi posteriormente aplicado em dados de PMUs instaladas no SIN com o objetivo de estimar os modos de oscilação dominantes do sistema. Todas as medições em PMUs foram realizadas em laboratórios de algumas universidades brasileiras, no lado de baixa tensão. O pré-processamento dos dados foi realizado utilizando interpolação e filtragem, de modo que restassem apenas as componentes de frequência do sinal na faixa de interesse. As medições, na forma sincronizada, ficam acessíveis em um concentrador de dados fasoriais (Phasor Data Concentrator - PDC), instalado na UFSC $[7,8,10]$.

\subsection{Publicação}

Os estudos desenvolvidos durante esta pesquisa possibilitaram a publicação de artigo em simpósio citado a seguir.

AMARAL, P. E. W. D.; FREITAS, F. D e FERNANDES, L. F. J. Estimação de Modo Dominante do Sistema Interligado Brasileiro via Filtro de Kalman Estendido usando Dados Ambientes Obtidos de Unidades de Medição Fasorial. In: XII SBAI - Simpósio Brasileiro de Automação Inteligente 2015, Natal-RN, disponível em http://swge.inf.br/SBAI2015/anais/446.pdf.

\subsection{Organização da Dissertação}

Seguindo a motivação e o objetivo, constantes do corrente capítulo, a presente dissertação possui a estrutura de acordo com a descrição a seguir.

O Capítulo 2 apresenta conceitos e fundamentos a respeito da estabilidade de SEP, apresentando, de forma geral, como são classificados os diversos tipos de problemas de instabilidade. Maior ênfase é dada aos problemas de instabilidade relacionados ao ângulo do rotor dos geradores síncronos, em especial aos estudos de estabilidade a pequenas perturbações, motivação do presente trabalho. É apresentado o referencial teórico para estudos de estabilidade a pequenas perturbações a partir do modelo linearizado de SEP, bem como da utilização de dados provenientes de PMUs de forma 
complementar a esses estudos. Por fim, este capítulo apresenta uma revisão bibliográfica a respeito da utilização de dados de ambiente coletados por meio de PMUs para identificação de modos de oscilação em SEP.

No Capítulo 3 são apresentadas informações sobre medidores fasoriais sincronizados, chamados de PMUs. São descritas as etapas do pré-processamento dos dados de PMUs, tais como interpolação e filtragem dos sinais. Neste capítulo também são descritos e deduzidos os algoritmos do Filtro de Kalman (FK) e do FKE, assim como a metodologia para a identificação de parâmetros desconhecidos na forma de variáveis de estado. Ao final é apresentada a modelagem utilizada neste trabalho para identificação dos modos de oscilação de SEP, assim como a forma de ajuste dos parâmetros iniciais do modelo para aplicação do FKE.

No Capítulo 4 são desenvolvidas simulações de sistemas elétricos cujos modos de oscilação são conhecidos, utilizando o software PST. São apresentados cinco casos simulados, com diferentes características dinâmicas, bem como os resultados obtidos da análise dos sinais simulados utilizando o método proposto nesta dissertação. São apresentados também os resultados aplicados a sinais de defasagem angular de tensão coletados nas PMUs da UFT, UFC, UFMA, UFSC, UFRGS, UNIPAMPA e UTFPR, com o objetivo de identificar o modo de oscilação Norte-Sul do SIN. Por fim, no Capítulo 5 são apresentadas as conclusões gerais e sugestões para trabalhos futuros que poderão contribuir com a continuidade deste trabalho científico. 


\section{Estabilidade de Sistemas Elétricos de Potência}

\subsection{Introdução}

Os SEPs são formados por cargas, linhas de transmissão e diversas centrais de geração de energia elétrica. O funcionamento adequado de um SEP pressupõe o atendimento contínuo das cargas com qualidade, sem interrupções.

Para que um SEP opere de maneira contínua e segura, ele deve ser estável. A estabilidade de um SEP é a habilidade deste sistema, para uma dada condição de operação, de reestabelecer um estado de equilíbrio após sujeitar-se a um distúrbio, de forma que praticamente todo o sistema permaneça intacto [1].

Neste capítulo são apresentados os tipos de estabilidade de SEP e é dada ênfase na modelagem linear para avaliação da estabilidade a pequenas perturbações. Ao final é apresentada uma revisão bibliográfica acerca de trabalhos científicos que tratam da avaliação da estabilidade de SEP com a utilização de medições fasoriais sincronizadas coletadas por PMUs.

\subsection{Classificação da estabilidade de SEPs}

A estabilidade de um SEP é uma característica única. No entanto, existem diversas formas com que este sistema pode se tornar instável. Historicamente, o maior problema em termos de estabilidade de SEP sempre foi a instabilidade transitória, provocada por grandes distúrbios no sistema. Com a evolução natural dos sistemas, o aumento do número de interconexões de grandes áreas produtoras e consumidoras de energia elétrica, aumento da complexidade dos sistemas de controle, inserção intensa de fontes de energia renováveis e a operação dos sistemas com carregamento elevado, surgiram dificuldades adicionais de controle dos SEPs. Desta forma, ferramentas de controle e monitoramento visando preservar a estabilidade do sistema são sempre benéficas $[1,2]$.

Para facilitar o estudo e a análise da estabilidade de um SEP, os problemas de instabilidade são classificados para que cada um seja analisado da melhor forma, com o grau adequado de detalhamento e com as ferramentas corretas, possibilitando a adoção 
das ações necessárias para mitigação dos seus efeitos. Uma das formas de classificar os problemas de estabilidade está ilustrada na Figura 2.1.

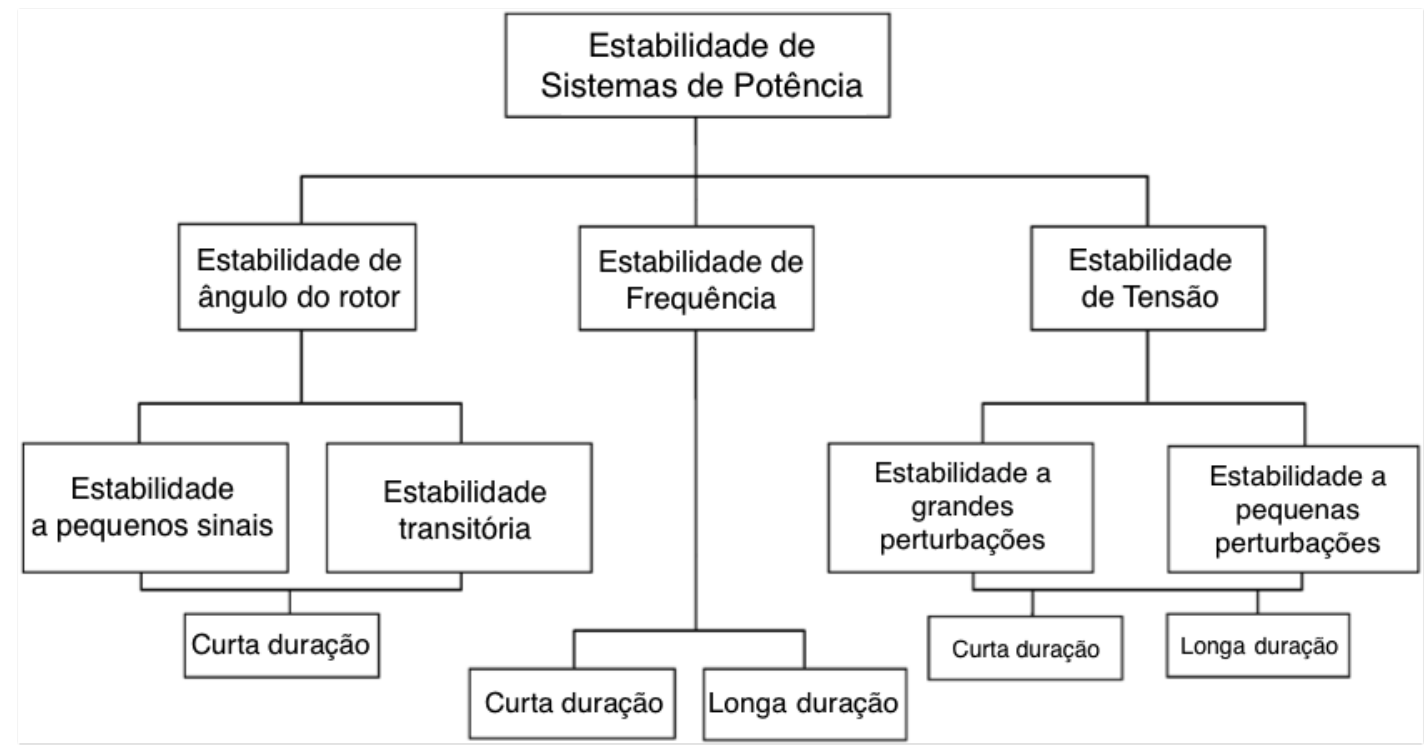

Figura 2.1: Classificação da estabilidade de SEP [1].

De acordo com esta classificação, os problemas relacionados a estabilidade estão divididos em três grupos: estabilidade de frequência, estabilidade de tensão e estabilidade do ângulo do rotor.

A estabilidade de frequência refere-se à capacidade de um sistema em manter a frequência nominal após um distúrbio severo que leve ao desequilíbrio entre geração e carga.

A estabilidade de tensão refere-se à capacidade de um SEP em manter as tensões nominais nas barras do sistema após um distúrbio. Geralmente os problemas de estabilidade de tensão estão associados ao comportamento das cargas frente a perturbações no sistema, aumentando a necessidade de potência reativa além da capacidade do sistema e ocasionando reduções nos níveis de tensão [1].

A estabilidade do ângulo do rotor refere-se à capacidade de os geradores síncronos de um sistema de potência permanecerem em sincronismo após uma perturbação no sistema. Este tipo de estabilidade está associada à capacidade de manutenção do equilíbrio entre o torque eletromagnético e o torque mecânico em cada gerador síncrono do sistema. Se um gerador tender a acelerar ou frear sua rotação, torques de sincronização irão tentar mantê-lo sincronizado [30]. Maior ênfase é dada a este tipo de estabilidade, em especial à estabilidade do ângulo do rotor a pequenas perturbações, por ser o objeto do presente estudo. 
Idealmente, existe equilíbrio entre o torque eletromagnético de saída e o torque mecânico de entrada em um gerador, de forma que a velocidade de rotação do rotor e do motor primário permanecem constantes. À medida que este equilíbrio do sistema é perturbado, os rotores das máquinas síncronas podem acelerar ou desacelerar de acordo com as leis de movimentos de rotação, alterando a posição angular do rotor de um gerador em relação à de outro. O rotor mais rápido passa a atender mais carga do que o rotor mais lento, e isto produz torques restauradores que tendem a reduzir a diferença de velocidade e a diferença angular entre os geradores. Entretanto, após um certo limite, o aumento na separação angular dos rotores reduz a transferência de potência, aumentando ainda mais a diferença angular. Assim, a estabilidade de ângulo do rotor do sistema depende da capacidade dos torques de restauração manterem os geradores em sincronismo. A perda de sincronismo pode ocorrer entre um gerador e o restante do sistema ou entre grupos de geradores, os quais podem permanecer em sincronismo entre si após um distúrbio que ocasione a divisão do sistema.

Os estudos de estabilidade de ângulo do rotor são subdivididos em duas categorias: estabilidade transitória e estabilidade a pequenas perturbações.

A estabilidade transitória trata da capacidade do SEP permanecer em sincronismo após grandes distúrbios, como, por exemplo, um curto circuito em uma linha de transmissão. Tais eventos envolvem grandes variações nos ângulos dos rotores dos geradores envolvidos e são influenciados pelo comportamento não linear da relação ângulo-potência.

A estabilidade a pequenas perturbações diz respeito à capacidade de um SEP manter o sincronismo quando submetido a pequenas variações de carga em um dado ponto de operação, de forma que a relação ângulo-potência pode ser considerada linear. Considerando que a carga do sistema está em constante variação e que os geradores síncronos possuem características e carregamentos diferentes, os modos de oscilação presentes no sistema estão constantemente sendo excitados [30, 31]. Assim, a análise da estabilidade de um SEP a pequenas perturbações é geralmente feita a partir de um modelo linearizado do sistema.

Esse tipo de problema de estabilidade está associado à insuficiência de amortecimento das oscilações eletromecânicas. Essas oscilações são características de sistemas elétricos, e podem ser amortecidas com a utilização de estabilizadores de sistemas de potência (Power System Stabilizers - PSS). Os PSSs fornecem sinais suplementares que são adicionados aos sistemas de excitação dos geradores síncronos. Além disso, as oscilações 
também podem ser amortecidas por meio de sinais suplementares em FACTS (Flexible AC Transmission System) [4].

A estabilidade a pequenas perturbações pode ser local ou global, envolvendo grandes áreas do sistema elétrico. Os problemas locais estão associados a oscilações entre um gerador síncrono e o restante do sistema ou entre geradores eletricamente próximos. Essas oscilações são chamadas de modos de oscilação locais e possuem frequências de oscilação na faixa de 1 a $2 \mathrm{~Hz}$.

Os problemas globais referem-se a interações entre grupos de geradores localizados em áreas diferentes de um SEP, e estão associados a linhas de transmissão de interligação fracas e carregadas entre as áreas. As oscilações, neste caso, são chamadas de modos de oscilação interárea [1], e possuem frequências típicas na faixa de 0,1 a $1 \mathrm{~Hz}$ [31]. Esse tipo de oscilação interárea tem se mostrado de grande importância à medida que os sistemas estão sendo interconectados por interligações com capacidade limitada e que operam próximas ao seu limite [31].

A Tabela 2.1 exemplifica as faixas de frequência em que estão localizados os principais modos de oscilação interárea presentes no SIN [9].

Tabela 2.1: Principais modos de oscilação interárea do SIN.

\begin{tabular}{c|c}
\hline Modo & Faixa de frequência \\
\hline Norte x Sul & $0,20-0,40 \mathrm{~Hz}$ \\
Sul x Sudeste & $0,60-0,80 \mathrm{~Hz}$ \\
Norte x Nordeste & $0,55-0,65 \mathrm{~Hz}$ \\
Mato Grosso x SIN & $0,40-0,45 \mathrm{~Hz}$ \\
Rio de Janeiro x SIN & $1,10-1,30 \mathrm{~Hz}$ \\
São Paulo x SIN & $0,65-0,75 \mathrm{~Hz}$ \\
\hline
\end{tabular}

A Figura 2.2 exemplifica um caso real no qual são ilustrados sinais coletados em PMUs que registraram oscilações pouco amortecidas. A figura apresenta o gráfico de potência ativa transmitida pela Linha de Transmissão Malin-Round Mountain 1, no oeste dos Estados Unidos. Este gráfico é amplamente divulgado em trabalhos científicos para descrever o blackout ocorrido em 1996 no sistema do WECC [32].

Neste gráfico observa-se que antes da abertura da Linha de Transmissão Keeler-Allston, em aproximadamente 400 segundos, o amortecimento do modo de oscilação interárea 


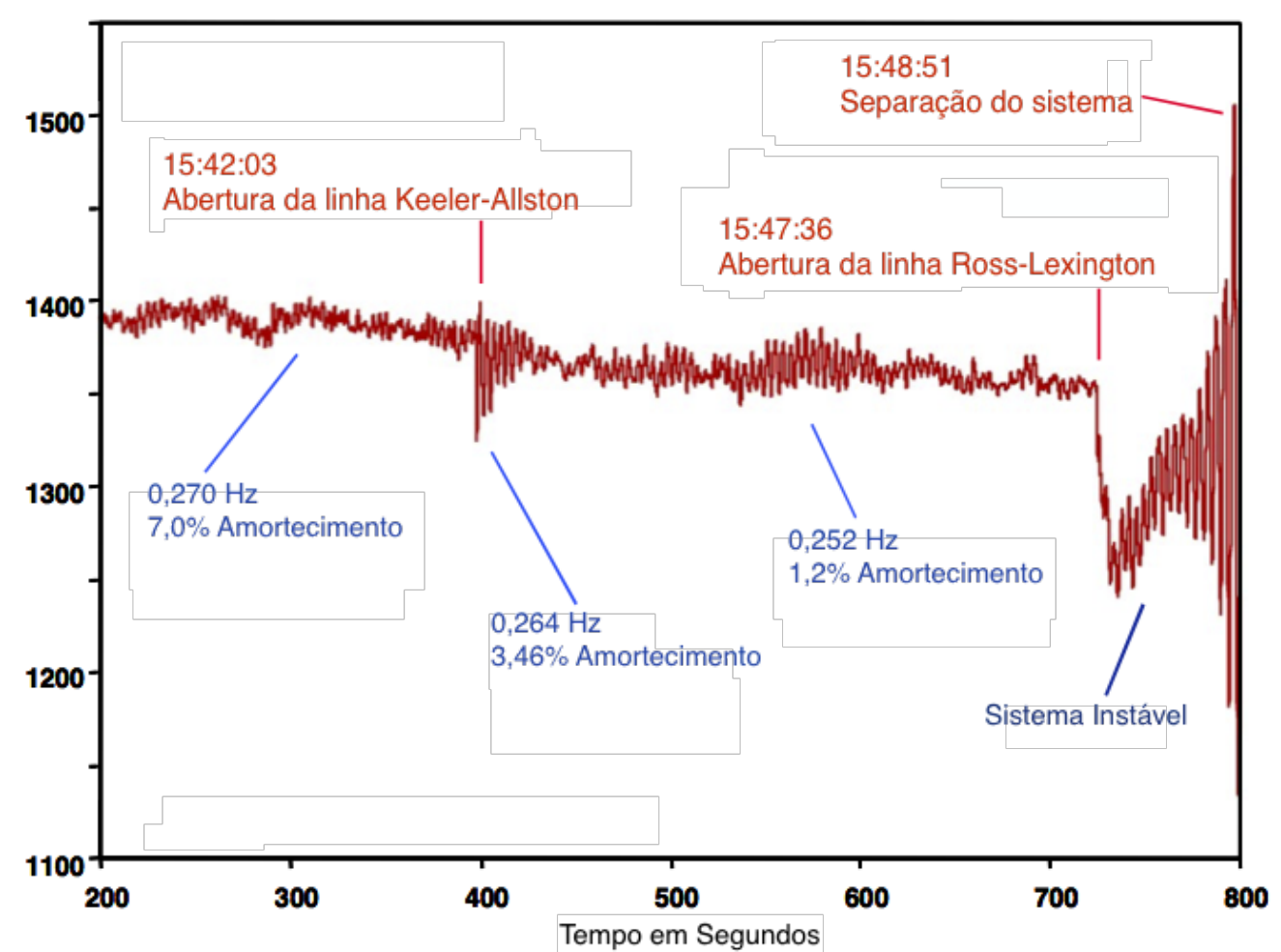

Figura 2.2: Fluxo de Potência na Linha de Transmissão Malin-Round Mountain 1 durante o blackout ocorrido no WECC em 1996 [2].

era de aproximadamente 7\%, considerado aceitável. A abertura desta linha, no entanto, tornou o sistema fraco, diminuindo o amortecimento do modo de oscilação para menos de $2 \%$. Posteriormente, a abertura da linha Ross-Lexington, aos 700 segundos, tornou o modo de oscilação interárea instável, o que levou o sistema ao colapso. Caso fosse possível a identificação do amortecimento dos modos de oscilação em tempo real, o operador do sistema poderia ter atuado com o objetivo de tentar atenuar ou mesmo evitar os problemas associados ao fraco amortecimento do modo de oscilação interárea.

Interligações que foram projetadas para uma certa capacidade, com o crescimento da demanda, são forçadas a operar próximas ao seu carregamento máximo e passam a se comportar dinamicamente como se fossem interligações fracas, sem condições de suportar as oscilações presentes no sistema. As oscilações ocasionam a flutuação da potência transmitida pelas linhas de transmissão, cuja proteção pode atuar em caso de excesso de fluxo, desligando a linha e isolando os sistemas [30].

\subsection{Modelo Linearizado de SEPs}

A estabilidade de um SEP a pequenas perturbações pode ser avaliada por meio do modelo linearizado do sistema para um dado ponto de operação, a partir da localização dos polos deste sistema no plano complexo. Se o modelo linearizado do sistema for 
estável, isso implica que o sistema é estável para aquele dado ponto de operação [6].

Os SEPs são modelados por conjuntos de equações algébricas e diferenciais não lineares (2.1), (2.2) e (2.3) [6,33].

$$
\begin{aligned}
& \dot{x}=f(x, z, u) \\
& 0=g(x, z, u) \\
& y=h(x, z, u)
\end{aligned}
$$

Incluem-se, por exemplo, as equações de oscilação das máquinas síncronas (2.4) e (2.5):

$$
\begin{gathered}
\frac{2 H}{w_{s}} \frac{d w}{d t}=P_{m}-P_{e} \quad \text { p.u. } \\
\frac{d \delta}{d t}=w-w_{s}
\end{gathered}
$$

onde $H$ é a constante de inércia da máquina síncrona, $\delta$ é o ângulo do rotor, $w$ é a velocidade angular do rotor, $w_{s}$ é a velocidade angular síncrona, $P_{m}$ é a potência mecânica entregue à máquina e $P_{e}$ é a potência elétrica entregue pela máquina.

Após a linearização das equações (2.1), (2.2) e (2.3) para um ponto de operação em regime permanente, são obtidas as seguintes equações na forma matricial:

$$
\left[\begin{array}{c}
\Delta \dot{x} \\
0 \\
\Delta y
\end{array}\right]=\left[\begin{array}{lll}
J_{f x} & J_{f z} & J_{f u} \\
J_{g x} & J_{g z} & J_{g u} \\
J_{h x} & J_{h z} & J_{h u}
\end{array}\right]\left[\begin{array}{c}
\Delta x \\
\Delta z \\
\Delta u
\end{array}\right]
$$

onde os elementos $J_{a b}$ são as submatrizes Jacobianas das funções $a$ em relação às variáveis $b$. Eliminando as equações algébricas, tem-se o chamado sistema "ABCD" de equações de espaço de estados que descrevem o modelo linearizado de um SEP.

$$
\begin{aligned}
& \Delta \dot{x}(t)=A \Delta x(t)+B \Delta u(t) \\
& \Delta y(t)=C \Delta x(t)+D \Delta u(t)
\end{aligned}
$$


onde a matriz $A$ é chamada de matriz de transição de estados, $B$ de matriz de entrada, $C$ de matriz de saída e $D$ de matriz de transmissão direta da entrada na saída [6]. Em modelos que descrevem SEPs a matriz $D$ é geralmente nula.

A partir do sistema de espaço de estados do modelo linearizado do SEP, os modos de oscilação são definidos pelos polos do sistema, expressos pelos autovalores da matriz de transição de estados $A[2,6]$. Para cada autovalor complexo $\lambda=\sigma \pm j \omega$, está associado um modo de oscilação com frequência de oscilação $\omega$ e taxa de amortecimento $\xi$, calculada em termos percentuais da seguinte forma:

$$
\xi(\%)=\frac{-\sigma}{\sqrt{\sigma^{2}+\omega^{2}}} \times 100
$$

Valores de taxa de amortecimento mínimo aceitáveis dependem de cada sistema elétrico e da sua operação. O valor típico mínimo aceitável é de 5\%. [31, 34].

O sistema somente será estável se todos os polos do sistema estiverem no semi-plano esquerdo do plano complexo, ou seja, se a parte real $\sigma$ de todos os polos tiver valor negativo. Se algum polo tiver parte real positiva, haverá no sistema uma oscilação senoidal não amortecida ou crescente, caracterizando um sistema instável. Caso exista algum polo situado sobre o eixo imaginário do plano complexo e todos os demais polos se situarem no semi-plano esquerdo, o sistema será dito marginalmente estável. Este tipo de sistema, no entanto, é de interesse apenas acadêmico [6].

Modelos lineares de SEP são formados por equações de espaço de estados de ordem elevada, com centenas de milhares de estados. No entanto, é possível a adequada caracterização do comportamento dinâmico do sistema com modelos de ordem reduzida, os quais são adequados também para o projeto de PSSs. Assim, na avaliação da estabilidade e no controle de SEP por meio da análise dos autovalores da matriz de transição de estados, geralmente são aplicadas técnicas diversas de análise de sistemas lineares esparsos e redução da ordem de modelos, preservando as principais características dinâmicas do sistema nas faixas de frequência de interesse $[2,12,13,14,15,16,17,18,19]$.

\subsection{Avaliação da estabilidade de SEPs utilizando medições fasoriais sin- cronizadas}

Tendo em vista a complexidade dos modelos matemáticos necessários para a avaliação da estabilidade de um SEP a partir da análise dos autovalores da matriz de transição 
de estados e que os parâmetros do sistema variam a todo instante, os dados de medição fasorial provenientes de PMUs têm sido empregados de forma complementar na identificação dos modos de oscilação, possibilitando uma análise quase em tempo real da estabilidade dos SEPs a pequenas perturbações [2, 20, 21, 22, 23]. Além disso, a análise de dados de PMU, por fornecer informações importantes do estado dinâmico do sistema em tempo real $[7,35,36,37]$, têm sido utilizada também em malhas de controle de PSS [38, 39].

Os sinais coletados por medidores fasoriais podem ser divididos em três tipos para efeitos de análise [2, 35]:

- Dados de ambiente: sinais de baixa energia provenientes da operação em regime permanente do sistema, produzidos por pequenas variações aleatórias de carga que excitam os modos de oscilação presentes;

- Probe signal: sinais transitórios gerados por meio da injeção de um sinal teste no sistema em estudo;

- Ringdown: sinais transitórios de alta energia ocasionados por algum distúrbio no sistema, curto-circuito, abertura de linha, etc., sobrepostos aos sinais de dados de ambiente.

Os sinais de ringdown e probe signal são caracterizados por terem alta energia e por apresentarem os modos de oscilação do sistema com maior intensidade que os dados de ambiente [34]. O comportamento do sistema, quando ocorre algum distúrbio ou injeção de sinal de teste, pode assemelhar-se à resposta de sistemas a uma entrada impulso ou degrau. Assim, o sinal normalmente pode ser analisado como um somatório de senoides amortecidas a partir de amostras de curta duração, da ordem de 20 segundos. Existe vasta literatura tratando da análise da estabilidade de SEP utilizando sinais de ringdown e probe signal [3, 22, 24, 25, 26, 27, 28, 29], e o método mais utilizado é o Método de Prony [2, 8, 35, 40, 41, 42, 43]. Esses sinais, no entanto, não estão disponíveis a qualquer tempo para que sejam continuamente utilizados para avaliação da estabilidade de sistemas.

Os dados de ambiente, por sua vez, são sinais com baixa energia, produzidos por pequenas variações aleatórias na carga e na geração do sistema, assumidas como de distribuição normal, mas suficientes para excitar os modos de oscilação presentes [11, $44,45]$ e fazer variar as grandezas do sistema, tais como tensão, corrente e frequência. Para analisar este tipo de sinal são necessários períodos amostrais muito maiores, da 
ordem de 10 minutos, para que os resultados sejam adequados [34]. Não obstante, não são exatos devido à natureza estocástica do comportamento do SEP [5, 32]. No entanto, diferente dos sinais de ringdown, os sinais de dados de ambiente estão disponíveis para análise todo o tempo, menos nos instantes em que ocorrem distúrbios, abrindo a possibilidade para o monitoramento em tempo quase real dos modos de oscilação dominantes no sistema.

Nas análises de sinais de dados de ambiente, considera-se que o modelo linearizado do SEP é da forma das equações de espaço de estados de um sistema autônomo estocástico $(2.10)$ e $(2.11)$ a seguir $[4,2]$ :

$$
\begin{gathered}
\dot{x}(t)=A x(t)+w(t) \\
y(t)=C x(t)+v(t)
\end{gathered}
$$

onde os vetores $w(t)$ e $v(t)$ representam ruídos, a exemplo da variação aleatória da carga caracterizada como um ruído branco de baixa amplitude excitando o sistema, e os ruídos presentes no processo de medição fasorial, respectivamente.

Os sinais utilizados neste trabalho, para identificação de modos de oscilação dominantes em SEP, foram dados de ambiente simulados através do software livre PST e dados coletados de medidores fasoriais instalados no âmbito do projeto Medfasee, da UFSC. As principais análises encontradas na literatura relacionadas a dados de ambiente utilizam como forma de encontrar os modos de oscilação de SEP os modelos Autoregressive (AR) e Autoregressive Moving Average (ARMA), métodos de YuleWalker [5, 11, 46, 34], Least Mean Squares (LMS) [47, 48, 49], Recursive Least Squares (RLS) [50, 51, 52], FK [53, 54], Transformada Wavelet e Random Decrement Technique (RDT) [45, 55, 56, 57], Recursive Maximum Likelihood [58], e métodos de subespaço de estados $[8,23,34,59,60,61,62,63]$.

\subsection{Revisão Bibliográfica}

Conforme conhecimento do autor, o primeiro trabalho a utilizar dados de ambiente medidos por meio de PMU para analisar os modos de oscilação eletromecânica presentes nos sistema elétricos foi [11]. A partir da publicação deste trabalho vislumbrou-se a possibilidade de identificação dos modos a partir de dados de ambiente. Até então, a análise restringia-se a sinais de ringdown. O autor deste trabalho assume que os dados 
de ambiente são gerados a partir de variações aleatórias das cargas, da geração e dos parâmetros do sistema que ocorrem continuamente e excitam os modos de oscilação. Assim, os valores de frequência e amortecimento dos modos podem ser estimados a partir do conteúdo espectral dos dados de ambiente. Essa hipótese é considerada por praticamente todos autores que analisam dados de ambiente.

Para fazer a análise, os autores de [11] utilizam um filtro branqueador. As variações aleatórias das cargas são caracterizadas como ruído branco Gaussiano (distribuição normal) que, ao excitarem o sistema, geram ruído colorido. O processo de filtragem do ruído colorido é feito por um filtro denominado branqueador, fornecendo na saída, a exemplo da entrada, ruído branco. A Figura 2.3 ilustra o diagrama de bloco geral deste tipo de filtro. O filtro branqueador baseia-se na função de autocorrelação dos dados

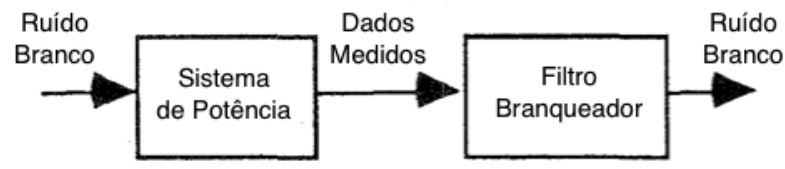

Figura 2.3: Filtro branqueador [11].

de ambiente, no método de Yule-Walker e na resolução das equações de Wiener-Hopf. As raízes do polinômio característico encontrado para este filtro representam os modos de oscilação do sistema. Os dados de ambiente analisados neste trabalho foram os de fluxo de potência em linhas de transmissão localizadas no oeste dos Estados Unidos. Os resultados encontrados foram comparados com aqueles encontrados utilizando o Método de Prony para sinais de ringdown de um sistema com as mesmas condições de operação dos dados de ambiente. Os resultados encontrados utilizando os dados de ambiente e de ringdown foram consistentes.

Em [47], os autores de [11] utilizaram o método LMS para criar um filtro adaptativo. Neste caso foi utilizado um algoritmo de LMS adaptativo para calcular os parâmetros do filtro de Resposta ao Impulso Finito, a partir dos quais são calculados os modos de oscilação identificados no sinal. Desta forma, as variações nos modos de oscilação do sistema simulado puderam ser avaliadas ao longo do tempo. Foram feitos testes com um sistema estacionário, um sistema estacionário com falta e com outro cujos modos de oscilação variam no tempo. Os resultados indicaram o potencial dos filtros adaptativos, como o LMS, para a identificação dos modos de oscilação em sistemas elétricos por meio de dados de ambiente. Um problema que o autor salientou foi quanto aos valores iniciais dos parâmetros do filtro LMS que devem ser próximos aos valores corretos para que ocorra a convergência. Em [49], os mesmos autores propuseram uma 
combinação de métodos baseados em LMS com parâmetros adaptativos com o objetivo de diminuir a variabilidade e o tempo de convergência das estimativas de frequência e amortecimento.

Em [46] os autores analisaram os dados de ambiente a partir de modelos AR e ARMA. Neste trabalho os parâmetros da equação característica dos modelos AR e ARMA são determinados por meio do método Yule-Walker Modificado aplicado a blocos de sinais. A partir destes parâmetros, é possível calcular as raízes da equação característica, as quais representam os polos do sistema elétrico, que por sua vez identificam a frequência e o amortecimento dos modos de oscilação presentes. Os resultados encontrados apontaram que os modelos AR e ARMA têm potencial para análise dos modos de oscilação com base em dados de ambiente de sistemas de potência.

Em [48], os autores de [47] e [46] combinaram as técnicas de filtros adaptativos LMS e modelos AR e ARMA para identificar os modos de oscilação em sinais simulados e em sinais ambiente de fluxo de potência medidos no sistema elétrico norte americano.

Em [50] e [51], os autores propõem métodos recursivos de análise de sinais. O primeiro trabalho apresenta o algoritmo denominado Robust Recursive Least Squares (RRLS) associado a um modelo AR, e o segundo apresenta o algoritmo denominado Regularized Robust Recursive Least Squares (R3LS) associado a um modelo ARMAX para identificação da frequência e amortecimento dos modos de oscilação em dados de ambiente, bem como ringdown e probe signal medidos por meio de PMU.

Em [34] e [59] são apresentados pelos autores métodos de identificação de sinais de dados de ambiente baseados em subespaços de estados, denominados N4SID e MOESP, processados em blocos de dados. Um método recursivo adaptativo baseado em subespaços de estados foi apresentado em [62].

O FK foi utilizado em [53] e [54] com o objetivo de identificar a frequência e o amortecimento de oscilações durante a operação normal do sistema elétrico, a partir de sinais de medição de PMUs e do modelo AR. Os modos de oscilação são encontrados a partir das raízes da equação característica do modelo AR. A partir de simulações, o autor selecionou sinais do sistema com alta observabilidade dos modos dominantes e demonstrou que o processo de identificação utilizando FK apresentou bons resultados mesmo na presença de ruído de medição. 


\subsection{Conclusão}

Neste capítulo foram apresentados os tipos de estabilidade de SEP com ênfase na estabilidade de ângulo do rotor associada a pequenas perturbações. Foram abordadas também as formas de avaliação da estabilidade por meio de modelos do sistema elétrico e utilizando medições fasoriais sincronizadas. Por fim, foi feito levantamento bibliográfico sobre os principais trabalhos científicos que tratam da identificação de modos de oscilação e avaliação da estabilidade de SEPs a pequenas perturbações utilizando dados de ambiente coletados por meio de PMUs.

Nos próximos capítulos é apresentada a modelagem baseada em FKE proposta nesta dissertação para identificação de modos de oscilação eletromecânica dominantes em SEPs. 


\section{Medições, Métodos e Modelagem}

\subsection{Introdução}

Neste capítulo é apresentada uma metodologia para identificação de modos de oscilação dominantes em SEPs a partir de dados de ambiente coletados por meio de PMUs.

Primeiramente, são apresentadas os unidades de medição fasorial sincronizada, chamadas de PMUs, e a forma de pré-processamento dos dados coletados por estes medidores. Posteriormente são descritas as origens e a formulação matemática do FK e do FKE, que são estimadores recursivos estocásticos de estados a partir de medições ruidosas.

Por fim, é apresentada a modelagem em sistema de espaço de estados proposta nesta dissertação para identificação de modos de oscilação dominantes de SEP utilizando o FKE e as formas de ajustes dos parâmetros iniciais do FKE.

\subsection{Unidades de Medição Fasorial Sincronizada}

As PMUs são equipamentos de medição fasorial sincronizada que mensuram e calculam os valores de módulo e fase da tensão e da corrente das três fases e também de frequência elétrica de SEPs [9]. As PMUs cujos dados foram utilizados neste trabalho foram instaladas no âmbito do Projeto de Pesquisa Medfasee, sob responsabilidade da Universidade Federal de Santa Catarina (UFSC) [7]. Essas PMUs foram instaladas em baixa tensão e podem coletar sinais sincronizados temporalmente por GPS a uma frequência de até 60 amostras por segundo, taxa de amostragem superior a do sistema SCADA (Supervisory Control and Data Acquisition).

O Projeto Medfasee teve início em 2003 e seus principais objetivos são o desenvolvimento de um Sistema de Medição Fasorial Sincronizada (SMFS) e de aplicações para a análise e monitoramento do SIN. Os dados coletados pelas PMUs são encaminhados via Internet ao PDC localizado na UFSC, que tem a função de receber e armazenar os dados enviados por todas as PMUs. A Figura 3.1 apresenta a arquitetura básica de um SMFS.

Atualmente, estão instaladas 22 PMUs em vários centros de pesquisa de universidades 


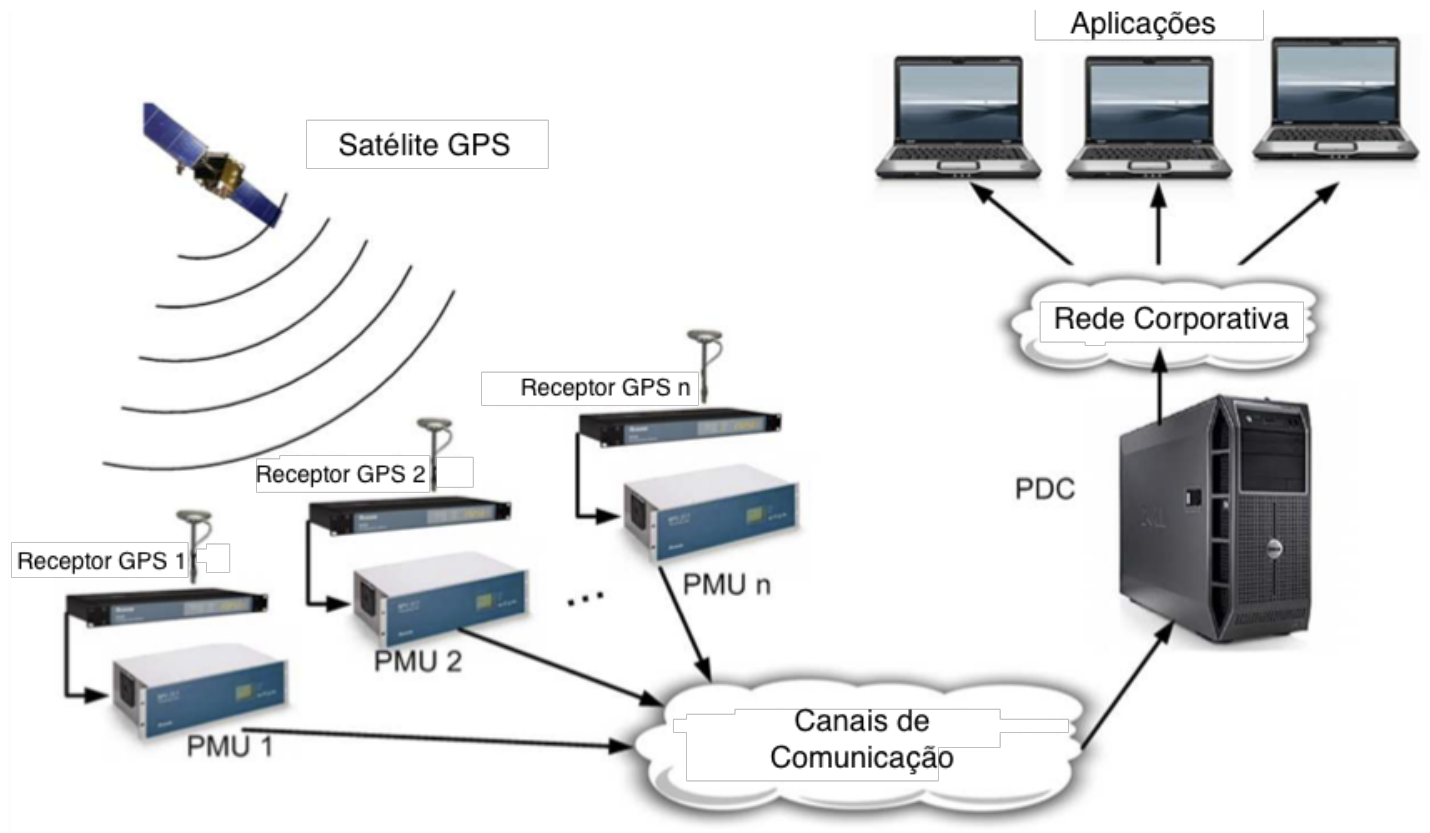

Figura 3.1: Arquitetura de um sistema de medição fasorial sincronizada [64].

em todas as cinco regiões geográficas do território brasileiro [10]. A Figura 3.2 ilustra o mapa do Brasil com a localização das PMUs atualmente implantadas.

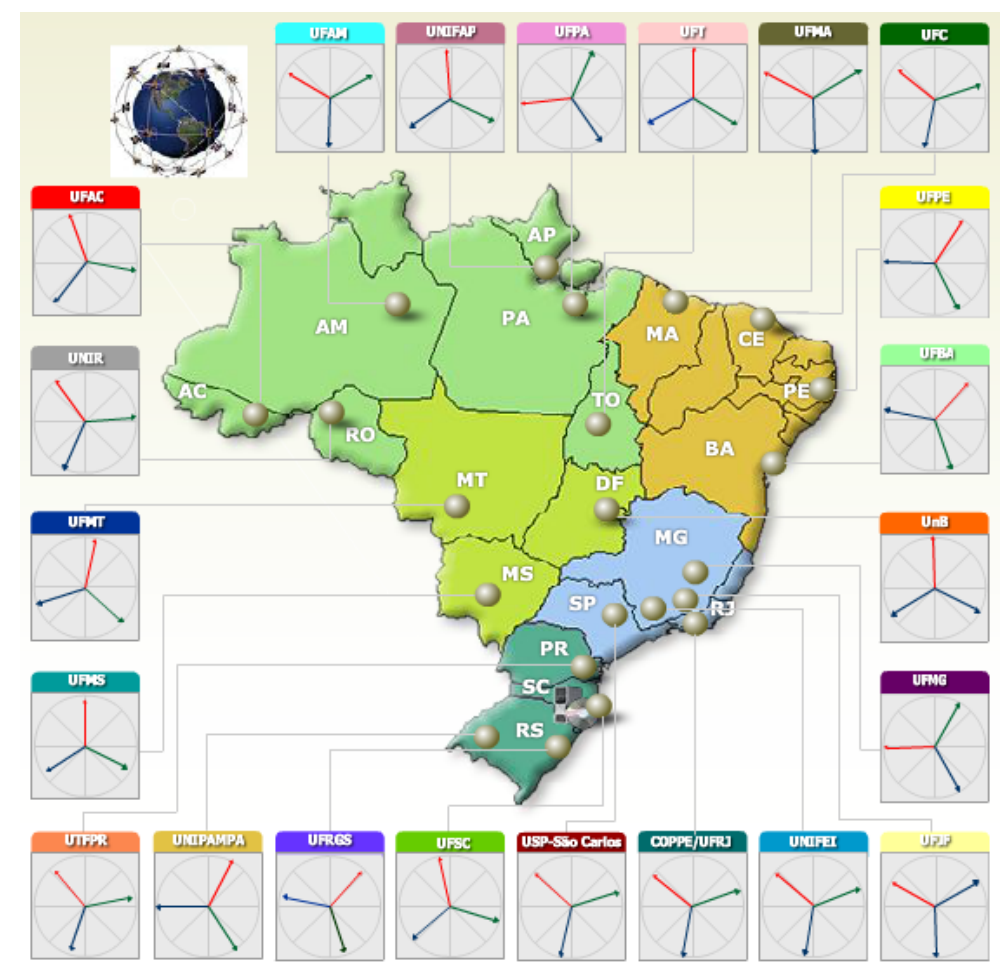

Figura 3.2: PMUs instaladas no âmbito do Projeto MedFasee [10].

\subsection{Pré-processamento de sinais}

Neste trabalho, optou-se por analisar os sinais de diferença angular das tensões de algumas PMUs instaladas no SIN, amostrados a uma taxa de 60 amostras por se- 
gundo $[8,44,65]$. Alguns estudos sugerem que esse tipo de sinal é melhor do que um sinal de ângulo absoluto para o controle de amortecimento de oscilações [32]. O sinal é inicialmente pré-processado para depois ser aplicado o algoritmo proposto neste trabalho para identificação de modos de oscilação dominantes presentes. Todo o préprocessamento bem como a implementação do algoritmo foram realizados utilizando o software Matlab.

Inicialmente, os arquivos de dados das PMUs são carregados e convertidos para o formato de arquivos que são lidos no Matlab. Posteriormente, os dados são interpolados, com o objetivo de atenuar distorções causadas por amostras corrompidas. Por fim, os valores dos ângulos são ajustados e o sinal é filtrado, de modo a atenuar as oscilações com frequência menor que $0,1 \mathrm{~Hz}$ e maior que $3 \mathrm{~Hz}$.

Alguns trabalhos que analisam sinais de PMU também promovem a redução da taxa de amostragem do sinal, principalmente aqueles que utilizam a matriz de autocovariância. Quando as taxas de amostragem são maiores do que $30 \mathrm{~Hz}$ a matriz de autocovariância pode ficar mal condicionada [32]. O algoritmo proposto neste trabalho, no entanto, não possui essa restrição e pode ser aplicado a sinais com amostragem de $60 \mathrm{~Hz}$, como é o caso dos sinais de PMU coletados.

As etapas do pré-processamento são descritas nas seções seguintes.

\subsubsection{Interpolação de sinais de PMUs}

Os dados coletados por PMUs podem apresentar amostras corrompidas com perda de informação, o que pode ocorrer por falha no equipamento bem como por falha na transmissão dos dados via Internet. Ao serem utilizadas em análises de fenômenos de sistemas elétricos, estas amostras corrompidas podem levar a resultados distorcidos.

Para preencher a amostra faltante, de modo que a taxa de amostragem dos dados permaneça constante, os aplicativos de busca de dados históricos de PMUs do projeto Medfasee repetem os valores da última amostra conhecida, até que novas medições sejam coletadas.

Como exemplo, a Figura 3.3 apresenta um trecho de dados de ângulo de tensão da fase A coletados da PMU instalada na UnB, no qual é possível observar dados corrompidos entre $2475 \mathrm{~s}$ e $2481 \mathrm{~s}$. 


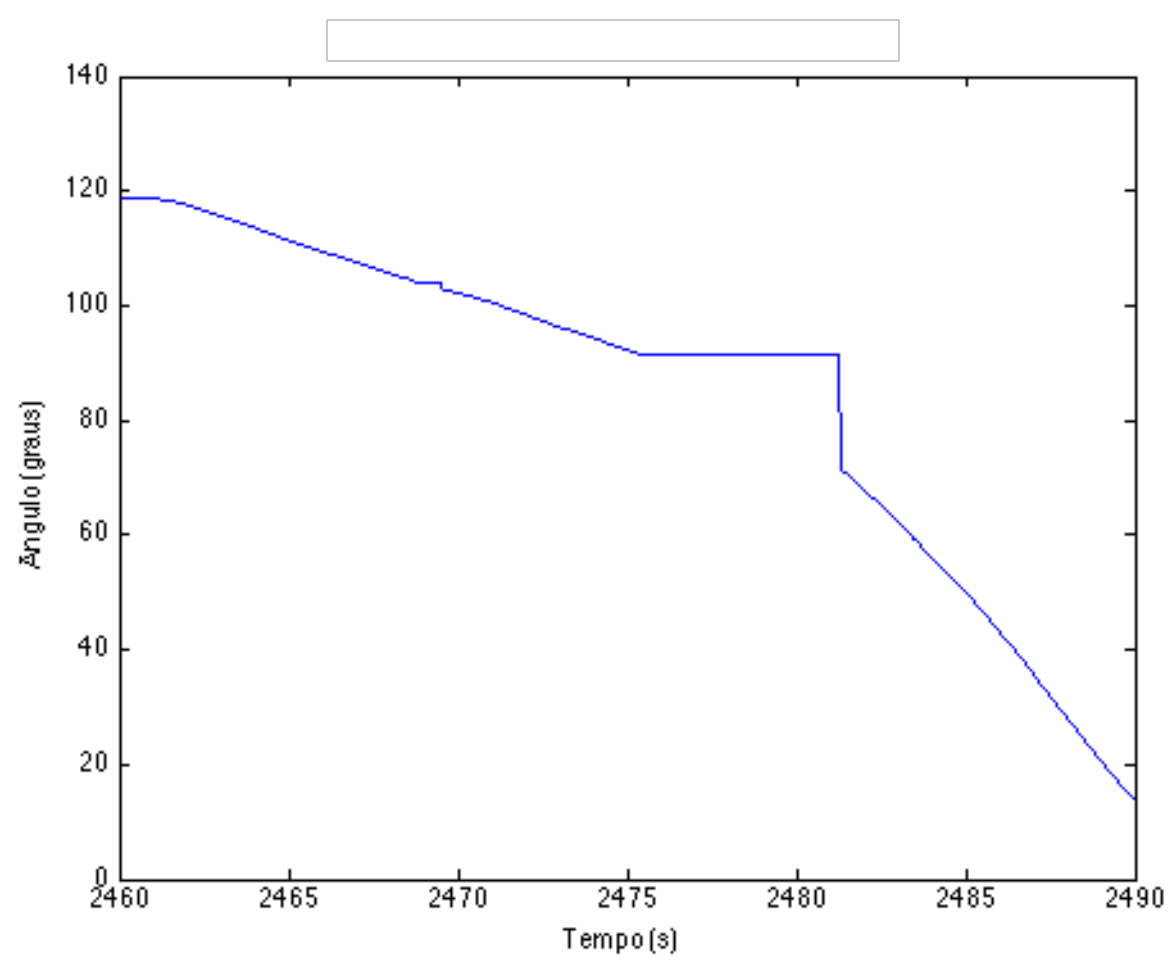

Figura 3.3: Ângulo da fase A do sinal de tensão da UnB

Com o objetivo de atenuar a distorção provocada pela perda de amostras, faz-se a interpolação de dados. Neste caso, as amostras corrompidas são substituídas por valores intermediários e inseridos como parte do sinal entre as amostras adjacentes efetivamente medidas.

Quando o número de amostras corrompidas é reduzido, a interpolação linear fornece bons resultados, sem perda relevante de informação. Entretanto, em algumas situações uma quantidade alta de amostras é perdida e a interpolação linear não é suficiente para atenuar a distorção do sinal, o que torna a interpolação mais complexa.

Caso estejam sendo analisados os sinais de diferença angular de tensão entre PMUs, a magnitude dos sinais será pequena. Nestes caso, se a interpolação não for realizada, ou se ela for realizada de forma indevida, este sinal de diferença angular de tensão entre PMUs ficará distorcido, com alguns valores muito superiores aos adjacentes, prejudicando os resultados da análise desejada.

Pode-se observar na Figura 3.4 o sinal de diferença angular de tensão na fase A entre os sinais das PMUs instaladas na UNB e na UFAC. Neste caso não é utilizada nenhuma técnica de interpolação, apenas os dados originais, sendo que os dados provenientes da PMU instalada na UnB estão corrompidos. 


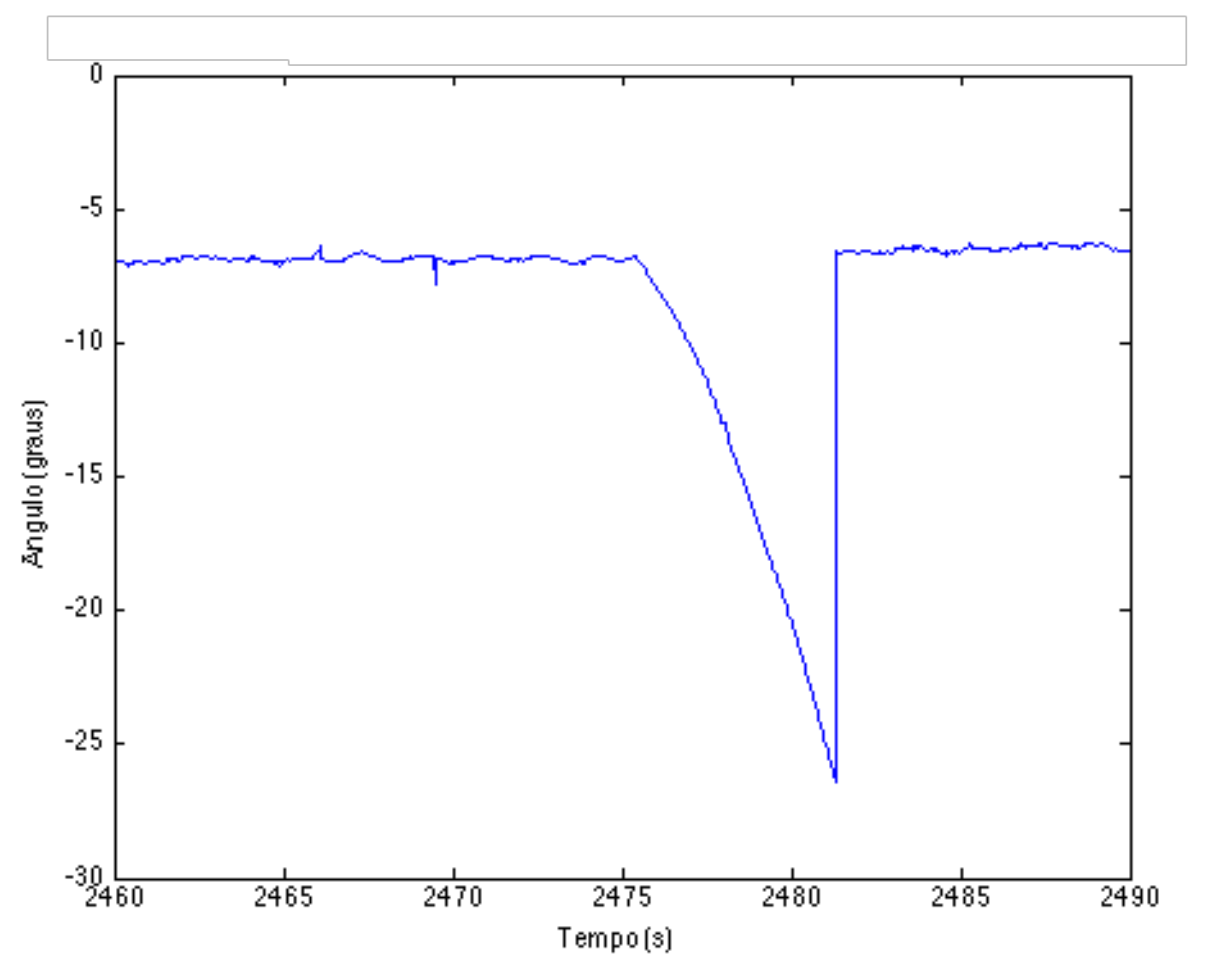

Figura 3.4: Diferença angular de tensão da fase A entre as PMUs instaladas na UnB e na UFAC

Verifica-se que, quando ocorrem dados corrompidos, a duplicação da última amostra conhecida pode gerar distorções nos sinais de diferença angular de tensão. No caso da distorção ilustrada pelas Figuras 3.3 e 3.4, foram registradas 354 amostras corrompidas, totalizando 5,9 segundos de dados.

A Figura 3.5 apresenta o mesmo gráfico da Figura 3.3 acrescido da curva com os dados obtidos por meio da interpolação linear.

É possível perceber que a interpolação linear achatou a curva do ângulo da tensão. Essa distorção fica clara no sinal de diferença angular de tensão da fase A entre os sinais de PMU da UnB e da UFAC, por exemplo, na Figura 3.6.

Observa-se que, no trecho entre 2475 s e 2481 s, a magnitude da diferença angular diminui significativamente, distorcendo o sinal. De fato, observando a Figura 3.7, que mostra a curva do ângulo da fase A da PMU da UnB com interpolação linear e a curva do ângulo da fase A da PMU da UFAC, percebe-se que no trecho onde foi feita a interpolação linear a diferença angular é menor que nas adjacências, conforme gráfico exposto na Figura 3.6, o que pode causar distorção.

Inicialmente, nesta dissertação, foram feitos testes utilizando a interpolação linear, 


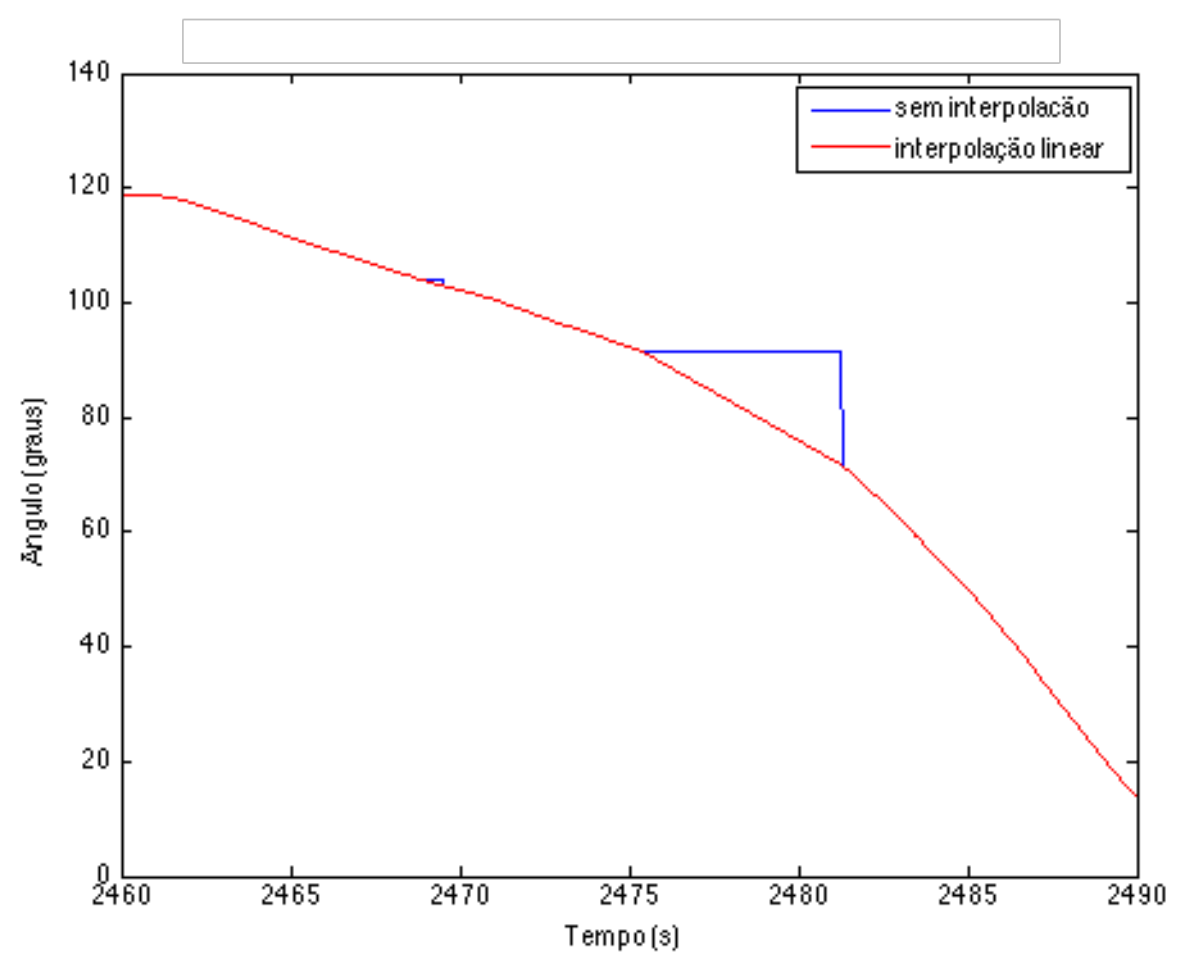

Figura 3.5: Ângulo da fase A do sinal de tensão da UnB sem interpolação e com interpolação linear

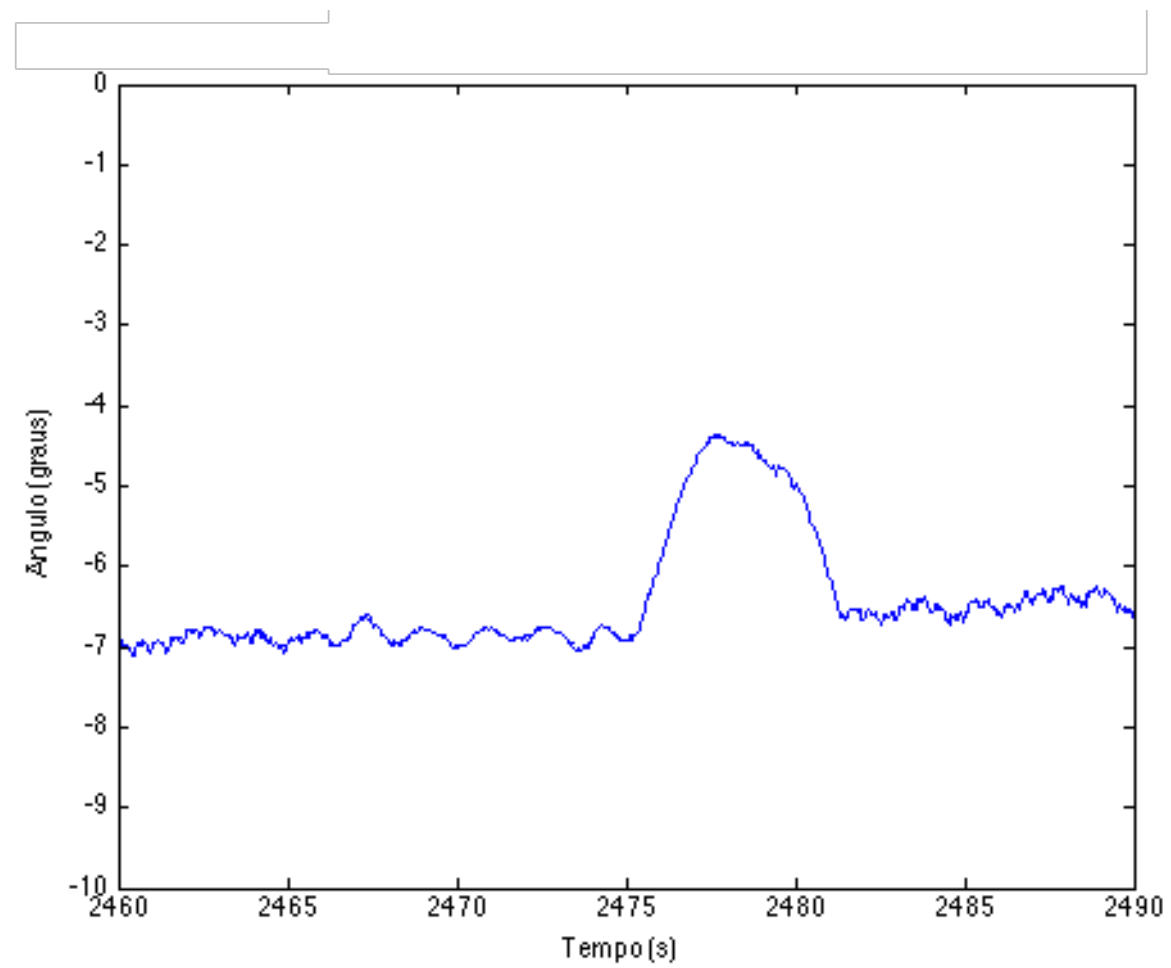

Figura 3.6: Diferença angular de tensão da fase A entre as PMUs instaladas na UNB e na UFAC utilizando interpolação linear

conforme sugerido em [66]. Observou-se, no entanto, que na análise de dados de diferença angular de tensão entre sinais coletados em duas PMUs distintas, devido à pequena magnitude do sinal, as distorções causadas pelas amostras corrompidas não 


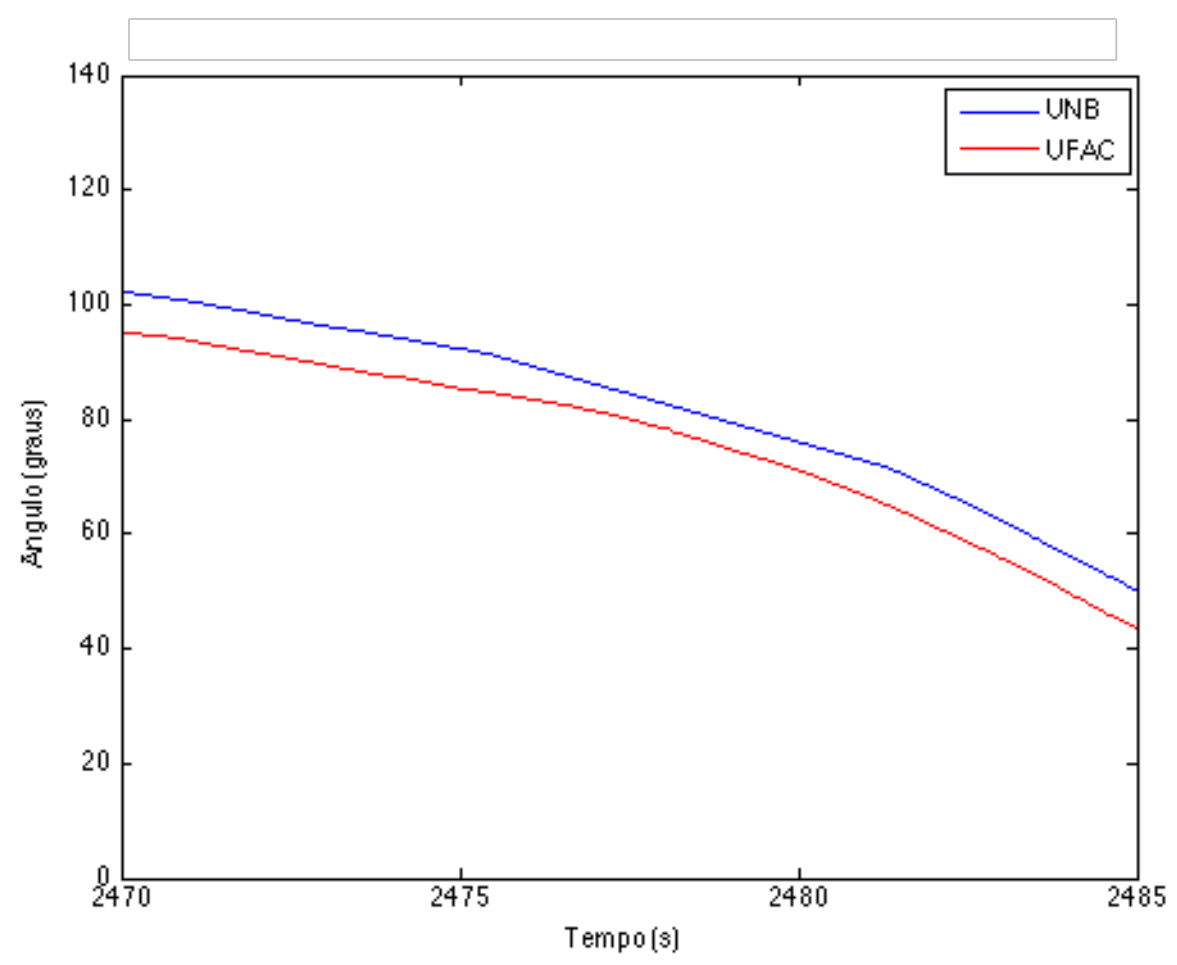

Figura 3.7: Ângulo de tensão da fase A das PMUs instaladas na UnB, com interpolação linear, e na UFAC, sem interpolação.

são satisfatoriamente atenuadas pela interpolação linear.

Com o objetivo de atenuar as distorções causadas por amostras corrompidas, optou-se, após estudos de alternativas, por utilizar a interpolação pelo método Piecewise Cubic Hermite Interpolating Polynomial-PCHIP [67], disponível no software Matlab, descrito na seção seguinte.

\subsubsection{Interpolação Piecewise Cubic Hermite Interpolating Polynomial-PCHIP}

A interpolação PCHIP é um método de interpolação cúbica monotônica por partes que preserva o formato da curva localmente, mantendo a primeira derivada contínua. Supondo que se queira interpolar uma função $f(x)$ em um intervalo $[a, a+n]$, a interpolação será monotônica se $f(i) \leq f(i+1)$, sendo $i=[a, a+1, a+2, \ldots, a+n-1]$, ou $f(i) \geq f(i+1)$, sendo $i=[a, a+1, a+2, \ldots, a+n-1]$. Assim, o objetivo da interpolação PCHIP é gerar uma função cúbica $p(x)$ que seja monotônica no intervalo entre $f(a)$ e $f(a+n)$.

Em cada subintervalo $\left[f_{i}, f_{i+1}\right], p(x)$ será um polinômio cúbico da seguinte forma:

$$
p(x)=f_{i} H_{1}(x)+f_{i+1} H_{2}(x)+d_{i} H_{3}(x)+d_{i+1} H_{4}(x)
$$


sendo que $d_{i}=p^{\prime}(i)$. As funções $H(x)$ são as funções cúbicas de Hermite, definidas da seguinte forma:

$$
\begin{gathered}
H_{1}(x)=\varphi\left(\frac{x_{i+1}-x}{h_{i}}\right) \\
H_{2}(x)=\varphi\left(\frac{x-x_{i}}{h_{i}}\right) \\
H_{3}(x)=-h_{i} \psi\left(\frac{x_{i+1}-x}{h_{i}}\right) \\
H_{4}(x)=h_{i} \psi\left(\frac{x-x_{i}}{h_{i}}\right)
\end{gathered}
$$

sendo que $h_{i}=x_{i+1}-x_{i}, \varphi(t)=3 t^{2}-2 t^{3}$ e $\psi(t)=t^{3}-t^{2}$.

Assim, o algoritmo de interpolação PCHIP irá determinar as derivadas $d_{i}, d_{i+1}, \ldots, d_{a+n}$, de tal forma que a interpolação seja monotônica, conforme detalhado em [67].

A Figura 3.8 apresenta uma comparação das curvas do ângulo de tensão da fase A da UNB utilizando a interpolação linear e a interpolação PCHIP. Observa-se que a interpolação PCHIP é mais suave do que a interpolação linear e preserva a curvatura do gráfico nas proximidades do trecho com amostras corrompidas. Os autores que desenvolveram este método dizem que é visualmente agradável (visually pleasing).

A comparação entre os sinais de diferença angular obtidos utilizando dados nos quais realizou-se interpolação linear e PCHIP pode ser observada na Figura 3.9.

Conforme pode ser visto na Figura 3.9, o sinal de diferença angular utilizando a interpolação PCHIP comporta-se de maneira semelhante ao esperado, tomando como base o comportamento do sinal onde não houve perda de dados, tornando imperceptíveis os dados corrompidos presentes no sinal de PMU da UNB.

As Figuras 3.10 e 3.11 ilustram o resultado de uma filtragem do sinal de diferença angular para atenuação das frequências inferiores a $0,1 \mathrm{~Hz}$, conforme é descrito na Seção 3.3.3, considerando a interpolação linear e PCHIP. Ilustra-se aqui o procedimento de filtragem passa-alta por ser processo muito utilizando em análise de sinais de PMU. 


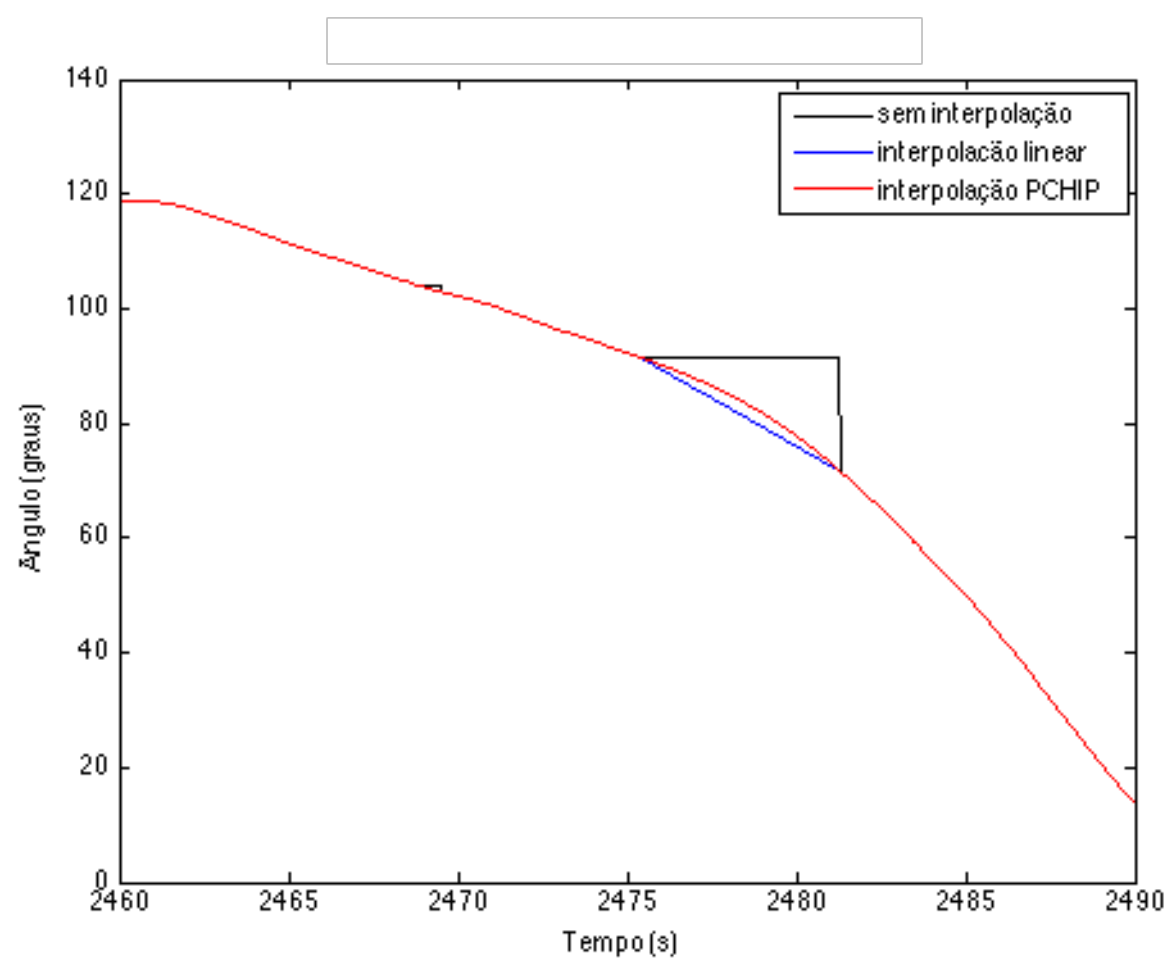

Figura 3.8: Ângulo de tensão da fase A da PMU instalada na UnB com interpolação linear e interpolação PCHIP

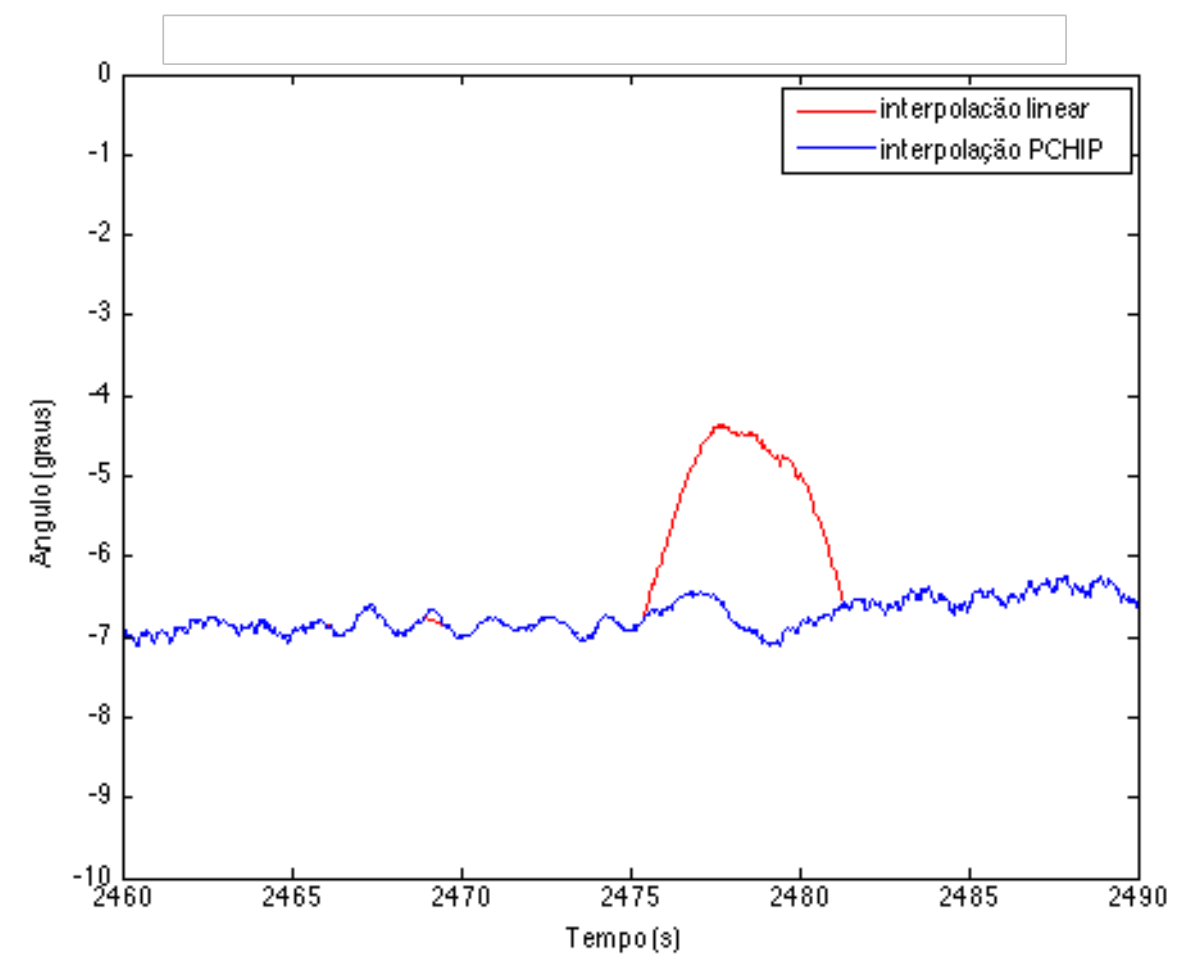

Figura 3.9: Diferença angular de tensão da fase A entre as PMUs instaladas na UnB e na UFAC utilizando interpolação linear e PCHIP

Percebe-se que a filtragem do sinal em que foi feita a interpolação linear gera uma pequena oscilação no sinal filtrado, o que não ocorre no caso da filtragem do sinal em que foi feita a interpolação PCHIP. 


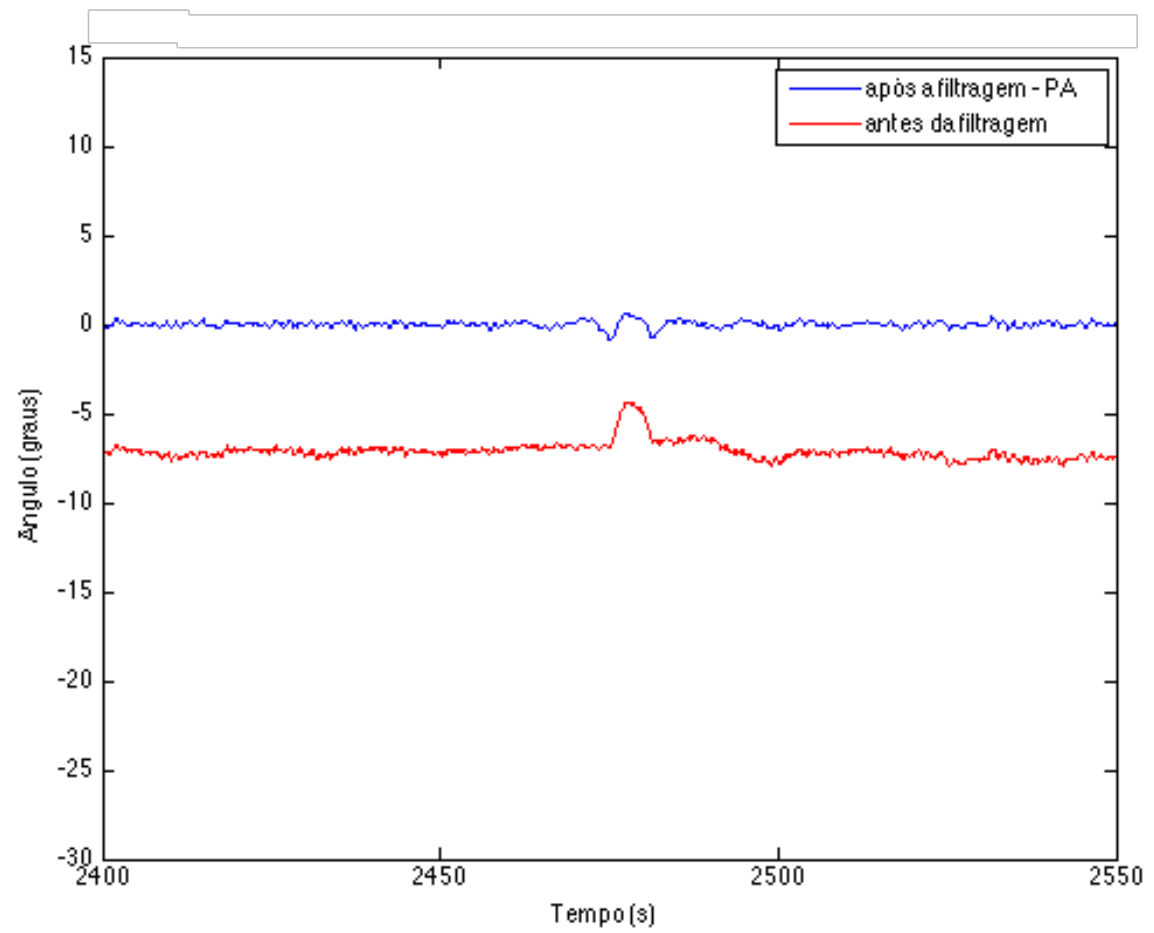

Figura 3.10: Diferença angular de tensão da fase A entre as PMUs instaladas na UnB e na UFAC, utilizando interpolação linear, antes e após a filtragem passa-alta

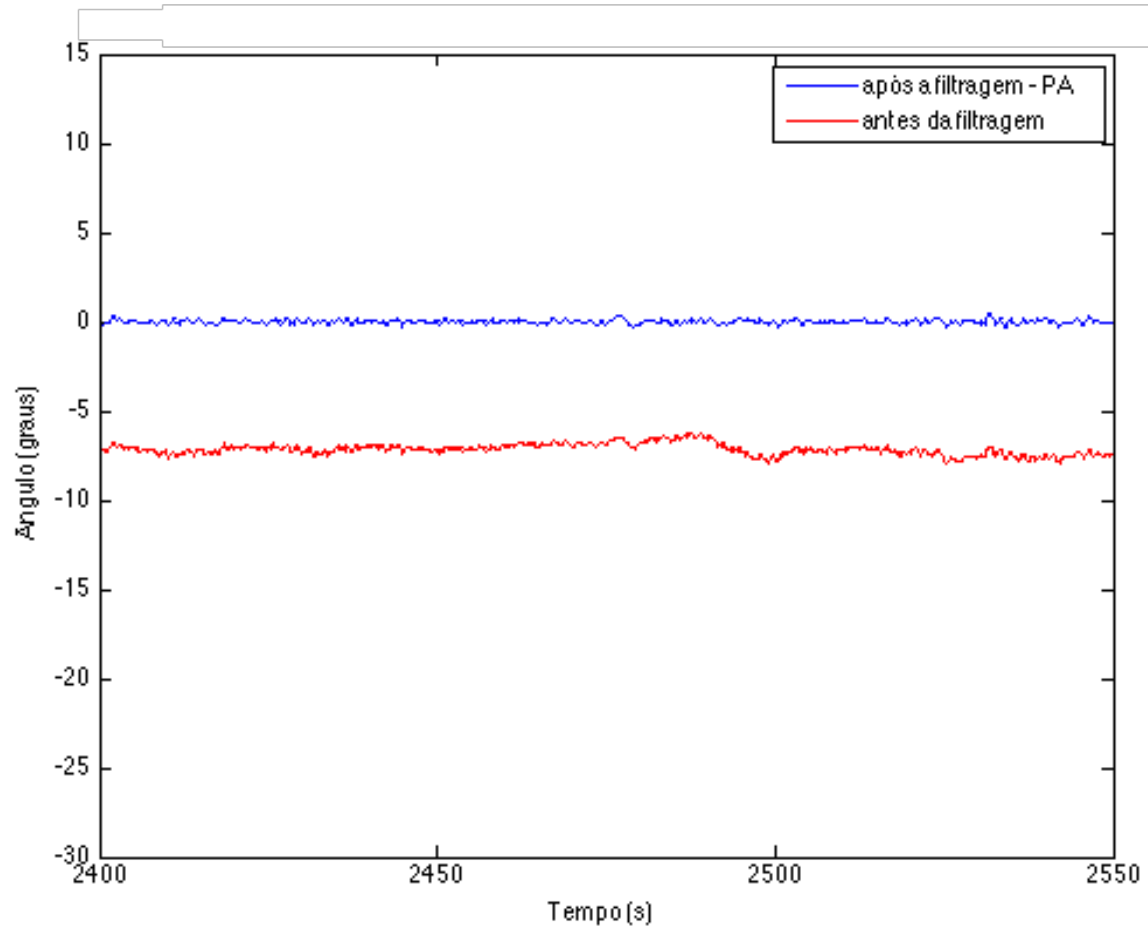

Figura 3.11: Diferença angular de tensão da fase A entre as PMUs instaladas na UnB e na UFAC, utilizando interpolação PCHIP, antes e após a filtragem passa-alta.

Apesar dos resultados positivos encontrados utilizando a interpolação PCHIP, em alguns casos, quando houver a perda de uma quantidade muito grande de amostras consecutivas, a interpolação PCHIP e tampouco a linear darão resultados adequados. 
A Figura 3.12 apresenta um trecho do sinal de ângulo de tensão da fase A da PMU localizada na UFRGS. Nesta ocasião ocorreram consecutivamente 2844 amostras corrompidas, aproximadamente 47,4 segundos de dados.

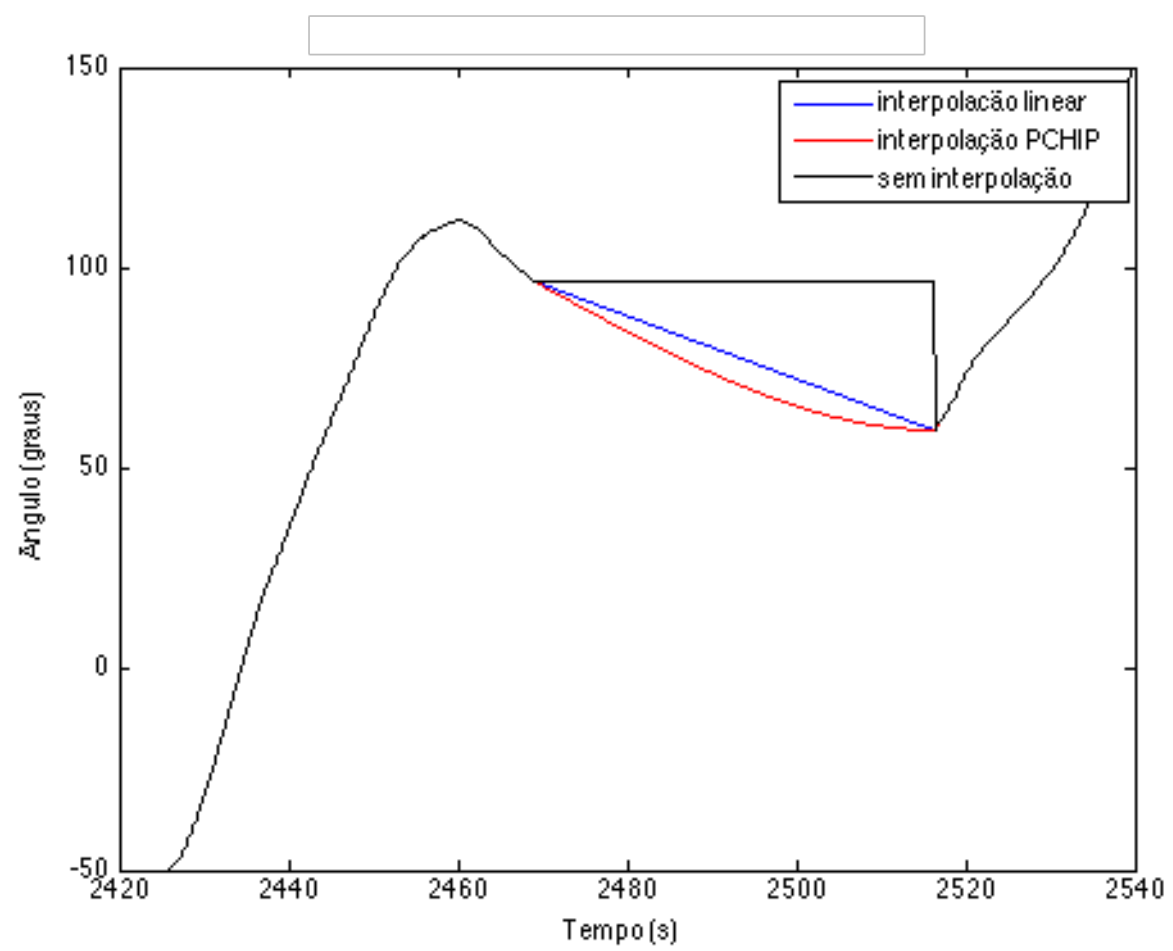

Figura 3.12: Ângulo de tensão da fase A da PMU instalada na UFRGS sem interpolação, com interpolação linear e com interpolação PCHIP

Observa-se na Figura 3.12 que as interpolações realizadas não foram adequadas para preencher as amostras corrompidas. Neste trecho do sinal, percebe-se que deveria haver um vale, no entanto, toda a curvatura foi perdida devido às amostras faltantes. Isso fica mais claro na Figura 3.13. Ela mostra os sinais de ângulo de tensão da fase A da PMU da UFRGS juntamente com o sinal de ângulo de tensão da fase A da PMU da UnB.

É possível observar que o sinal da UFRGS, mesmo após a interpolação, não acompanha a curvatura do sinal da UNB, como era de se esperar, tendo em vista que a diferença angular é pequena antes e depois do trecho com amostras corrompidas. Neste caso, uma outra estratégia para interpolação dos dados da PMU, ou exclusão dessas informações após certa quantidade de amostras seguidas corrompidas, deve ser investigada. No entanto, esta pesquisa não foi verificada nesta dissertação.

Conclui-se que a interpolação PCHIP foi mais eficaz que a interpolação linear como forma de atenuar as distorções causadas por amostras corrompidas nos sinais coletados em PMUs, principalmente quando forem analisados os sinais de diferença angular 


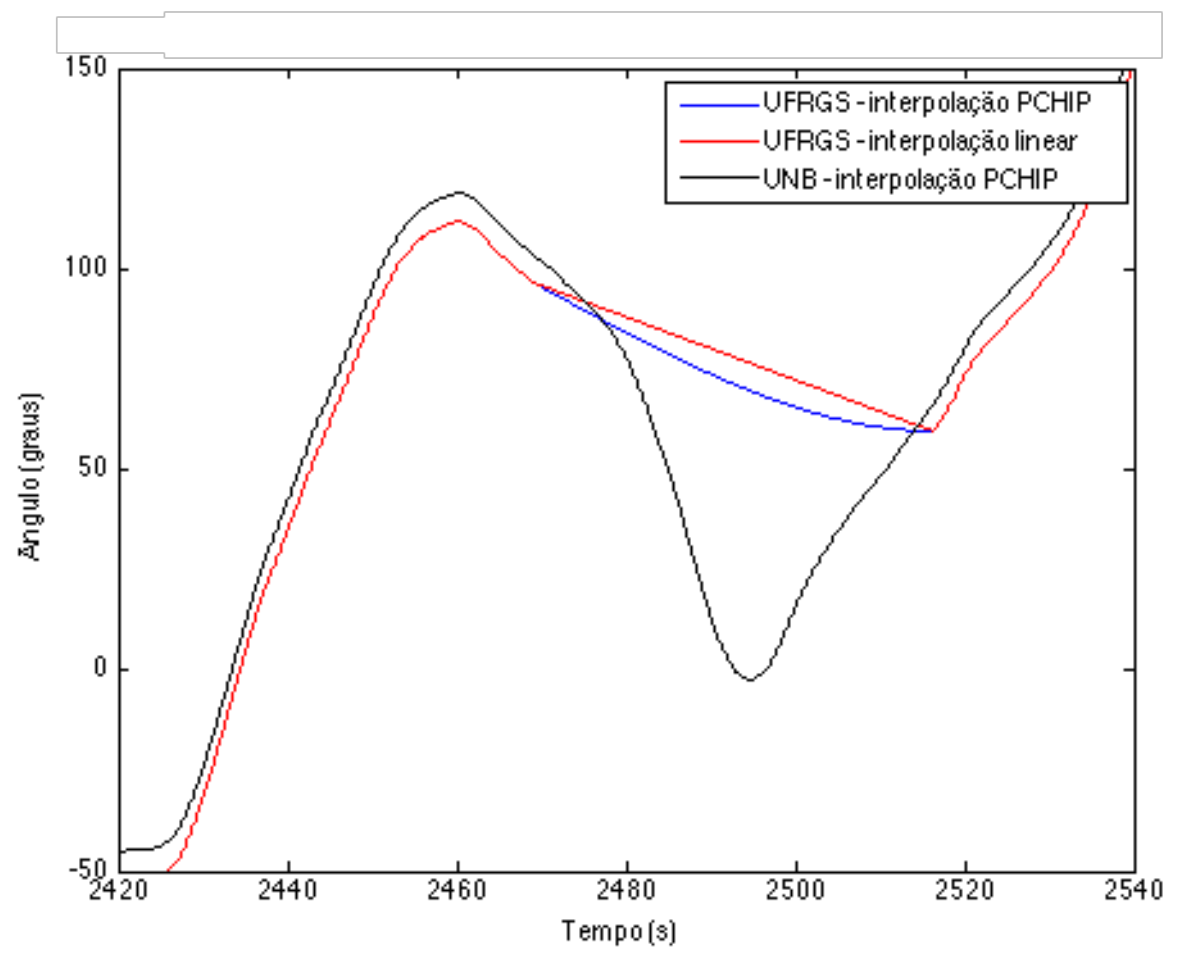

Figura 3.13: Ângulo de tensão da fase A das PMUs instaladas na UFRGS e na UnB com interpolação linear e PCHIP.

de tensão em sinais amostrados em diferentes localidades, pois estes sinais possuem pequena magnitude.

Apesar disso, observou-se que, quando a quantidade de amostras corrompidas consecutivas é muito grande, os resultados da interpolação PCHIP, assim como os da interpolação linear, tornam-se insatisfatórios. Esta observação era de se esperar, tendo em vista que, quanto maior a quantidade de amostras corrompidas, maior também será a perda de informações sobre o sinal.

\subsubsection{Ajuste dos valores de diferença angular}

Após a interpolação dos dados coletados das PMUs, são calculados os sinais de diferença angular de tensão entre duas localidades em análise.

Os valores dos ângulos absolutos das tensões nas barras do sistema podem aumentar ou diminuir conforme a frequência elétrica. A PMU, no entanto, registra os valores correspondentes limitados a uma volta do círculo trigonométrico entre $-180^{\circ}$ e $+180^{\circ}$. Ao proceder o cálculo da diferença angular de tensão entre PMUs pode acontecer de o ângulo de uma delas estar próximo de $+180^{\circ} \mathrm{e}$ o da outra de $-180^{\circ}$. A Figura 3.14 ilustra esta situação, na qual a diferença angular terá valores da ordem de 350 graus. 


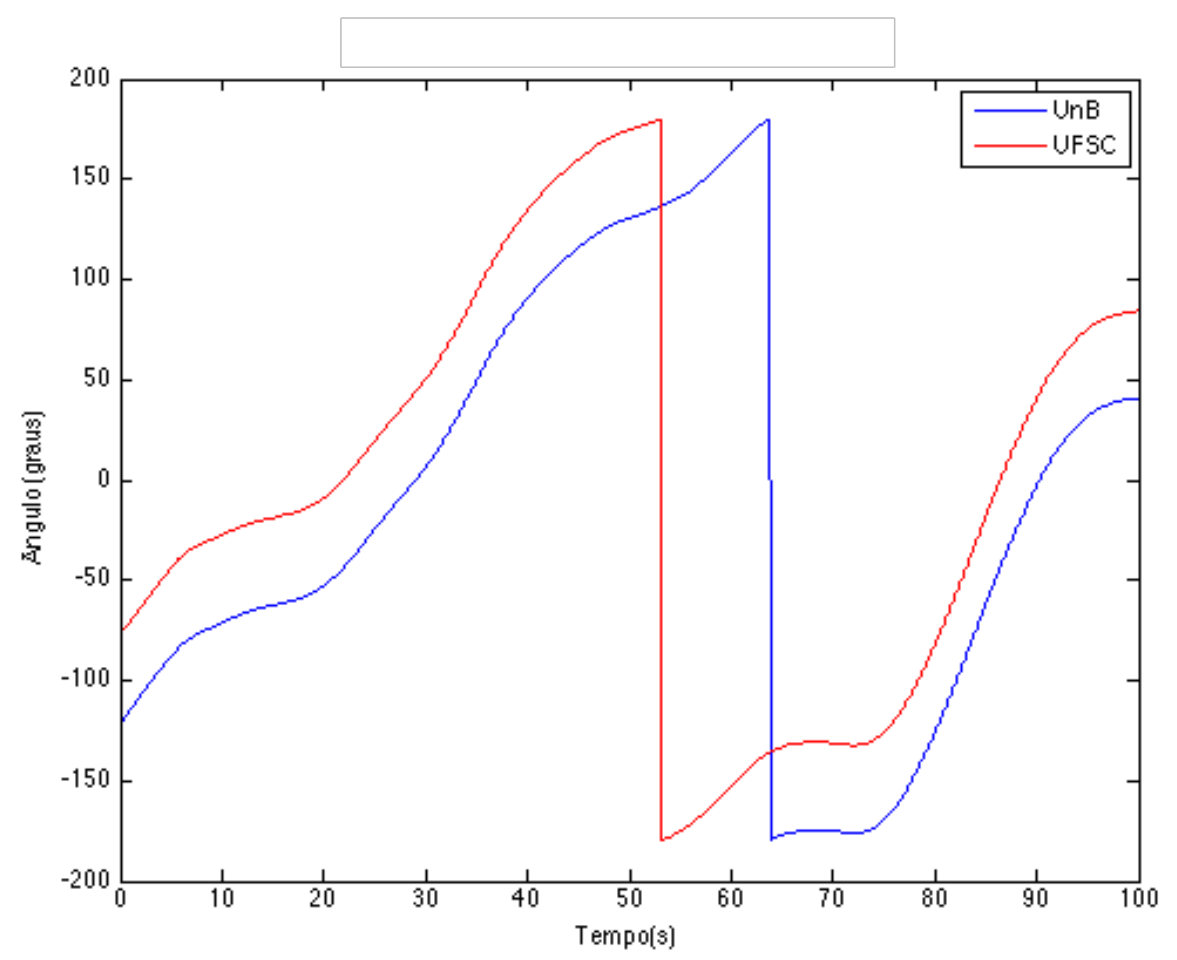

Figura 3.14: Ângulo de tensão da fase A das PMUs instaladas na UnB e na UFSC

A Figura 3.15 apresenta a curva de diferença angular de tensão para este caso. Observase que ocorre um acréscimo súbito nos valores calculados.

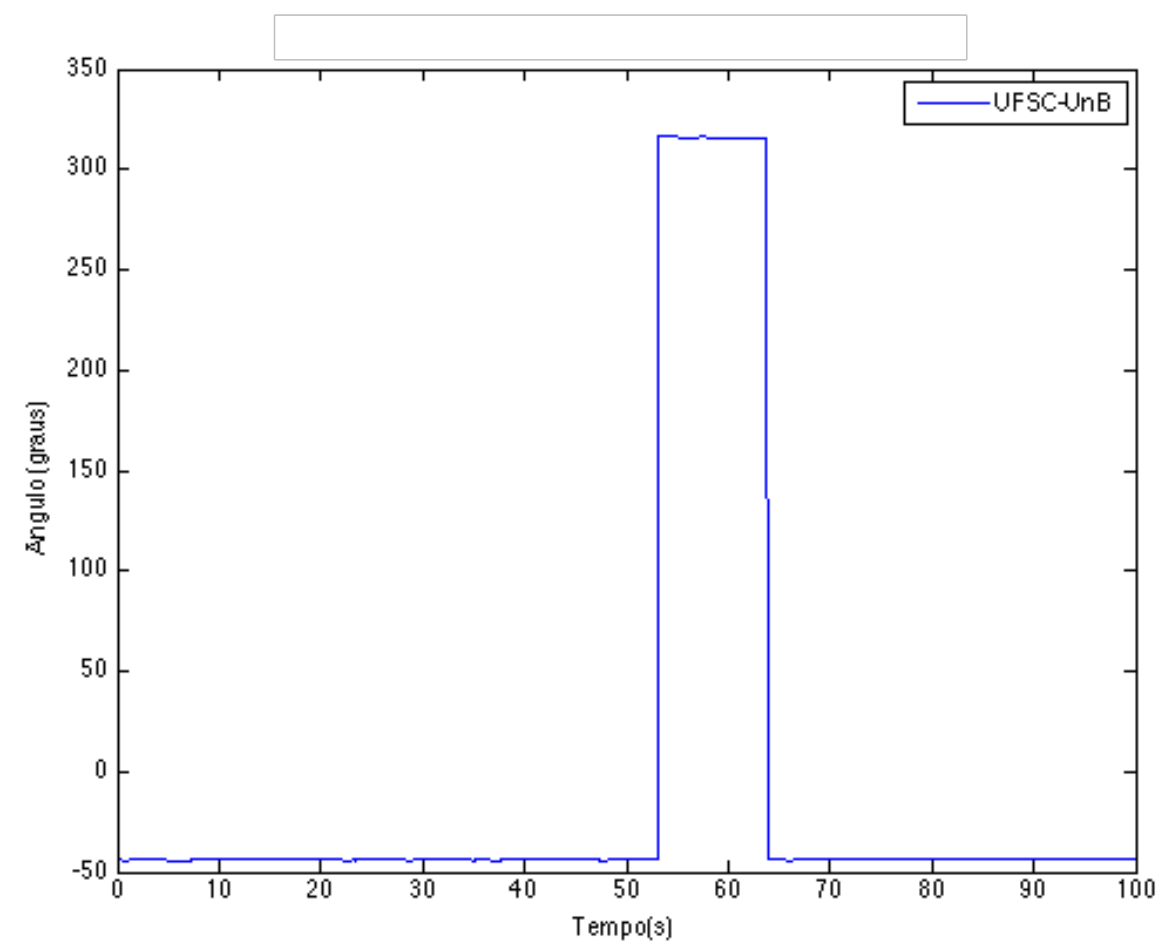

Figura 3.15: Diferença angular de tensão da fase A entre as PMUs instaladas na UnB e na UFSC antes do ajuste de valores angulares

Para corrigir esta situação, os valores de diferença angular de tensão que forem cal- 
culados e resultarem em valores superiores a 180, em módulo, são ajustados para os correspondentes valores angulares entre $-180^{\circ} \mathrm{e}+180^{\circ}$.

O exemplo acima de diferença angular de tensão entre as PMUs da UnB e da UFSC, após os ajustes dos valores, resulta na curva apresentada na Figura 3.16.

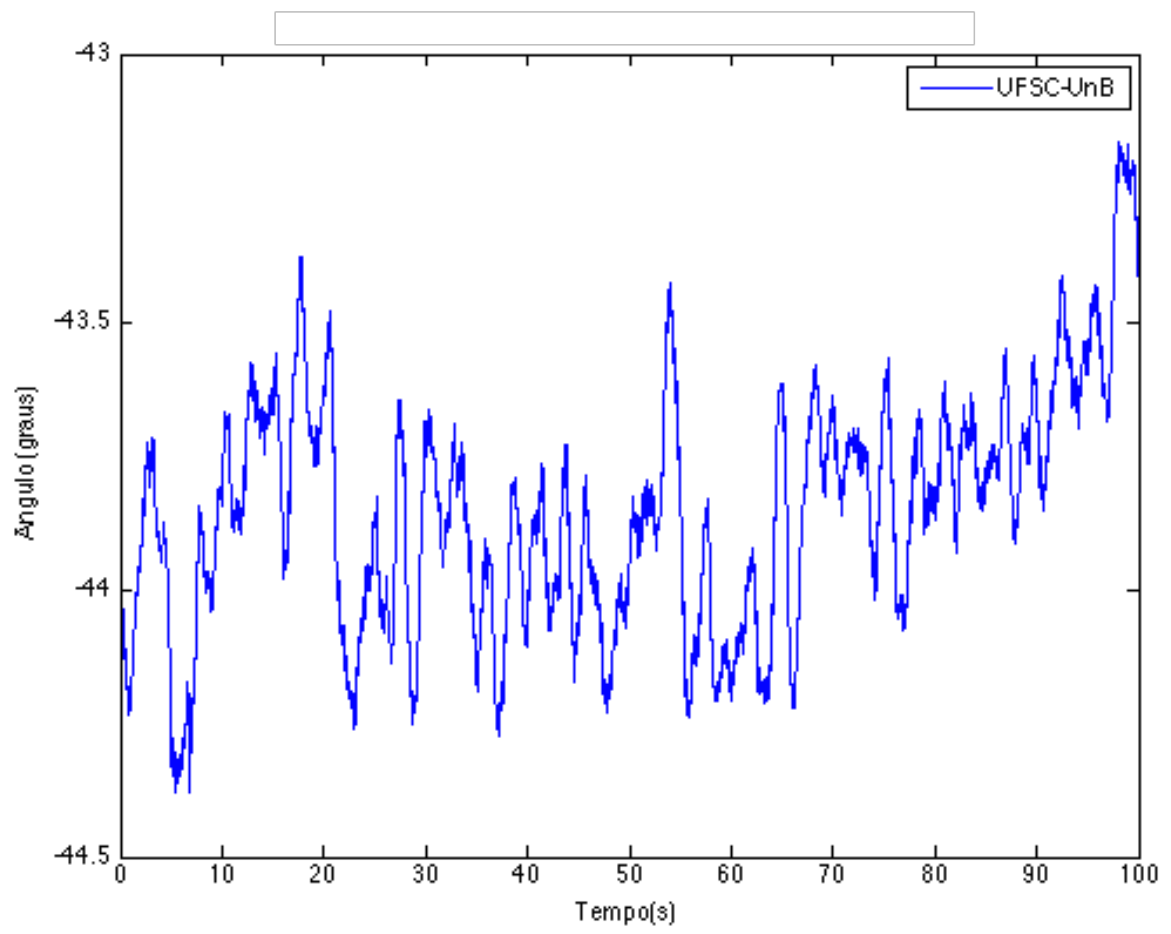

Figura 3.16: Diferença angular de tensão da fase A entre as PMUs instaladas na UnB e na UFSC após ajuste de valores angulares

\subsubsection{Filtragem dos sinais}

A última etapa do pré-processamento é a filtragem dos sinais de modo a atenuar as componentes com frequência menor que $0,1 \mathrm{~Hz}$ e maiores que $3 \mathrm{~Hz}$. Esta filtragem é necessária porque o objetivo é identificar oscilações eletromecânicas que, tipicamente, encontram-se na faixa entre $0,2 \mathrm{~Hz}$ e $2,5 \mathrm{~Hz}[5,11,53]$.

Com o objetivo de filtrar as frequências menores que $0,1 \mathrm{~Hz}$, foi utilizado um filtro digital de fase zero passa-alta de $20^{\mathrm{a}}$ ordem [5, 66]. Este filtro remove também as tendências existentes no sinal e a parcela determinística causada principalmente por reguladores de frequência no sistema. Antes de submeter o sinal ao filtro passa-alta, no entanto, remove-se a média do sinal para evitar o surgimento de oscilações na filtragem, de tal forma que o valor médio do sinal seja próximo de zero [66].

Além do filtro passa-alta, o sinal é submetido também a um filtro passa-baixa para 
remover as componentes de alta frequência do sinal, as quais não são objeto de estudo do presente trabalho por estarem fora da faixa de frequência das oscilações eletromecânicas.

Como exemplo, as Figuras 3.17 e 3.18 apresentam os espectros de frequências de um sinal de diferença angular entre as PMUs da UnB e da UFSC, com 1 hora de duração, antes e após a filtragem, respectivamente. A Figura 3.19 mostra a comparação entre os espectros de frequências antes e após a filtragem.

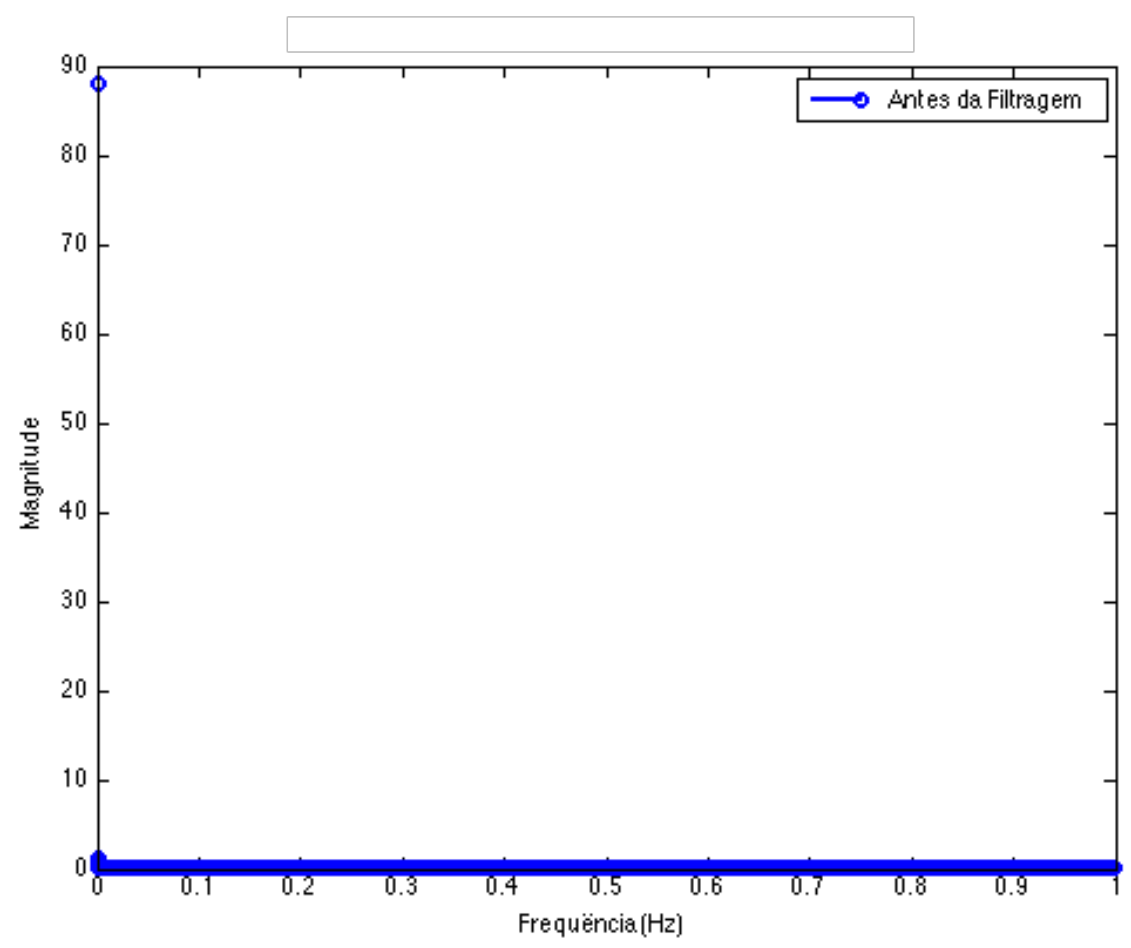

Figura 3.17: Espectro de frequências do sinal de diferença angular de tensão antes da filtragem

Percebe-se nos espectros das Figuras 3.17 e 3.19 que as componentes com frequência próxima a zero apresentam energia elevada em relação às demais componentes, o que pode dificultar a aplicação do algoritmo proposto neste trabalho. Após a filtragem e atenuação das frequências menores que $0,1 \mathrm{~Hz}$, constata-se que as oscilações na faixa de interesse entre $0,2 \mathrm{~Hz}$ e $0,8 \mathrm{~Hz}$ são aquelas com maior amplitude no sinal.

\subsection{Filtro de Kalman}

O desenvolvimento de ferramentas para processamento de dados contendo valores ruidosos iniciou-se com Gauss, no século XIX, que apresentou a técnica de mínimos quadrados [68], utilizada em problemas de estimação linear. Foi o primeiro método de estimação de parâmetros desconhecidos de modelos teóricos [69]. 


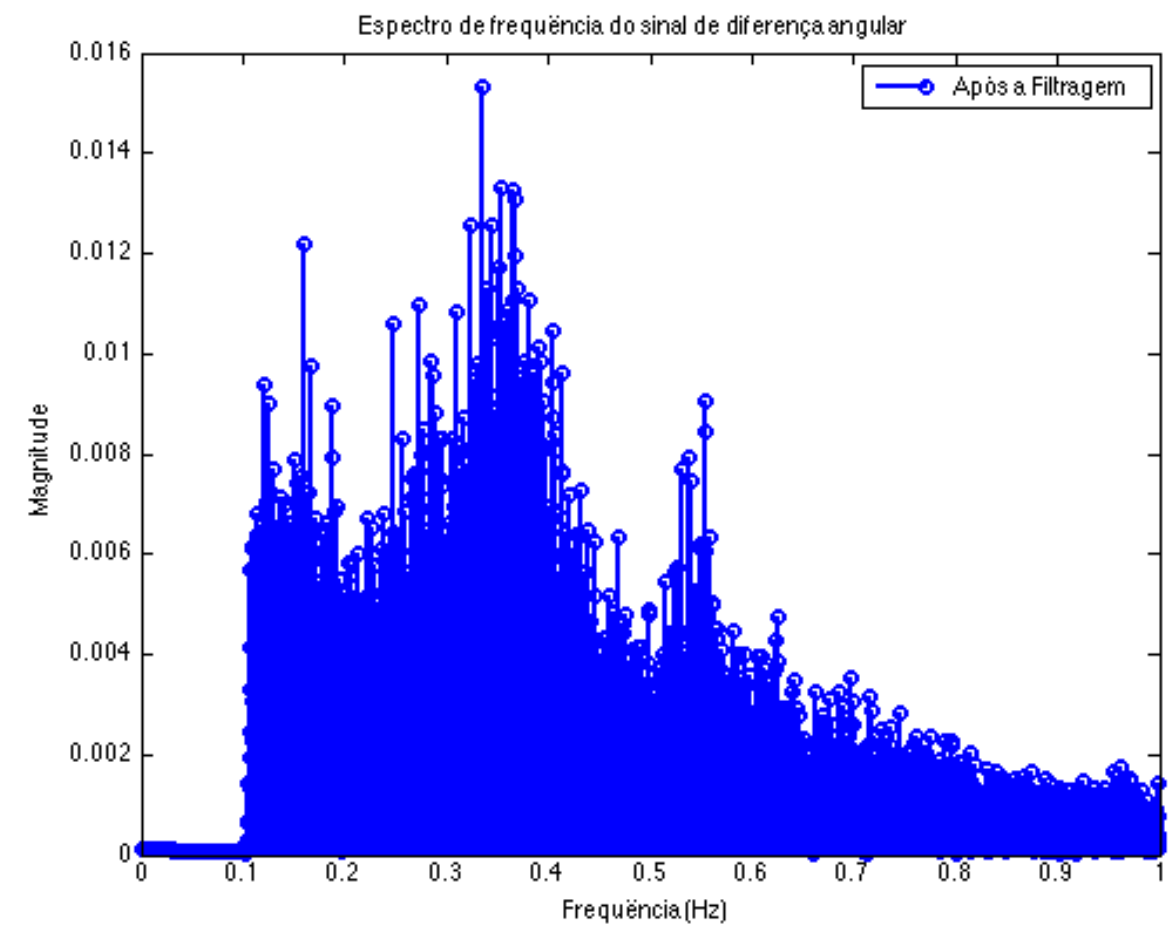

Figura 3.18: Espectro de frequências do sinal de diferença angular de tensão após a filtragem

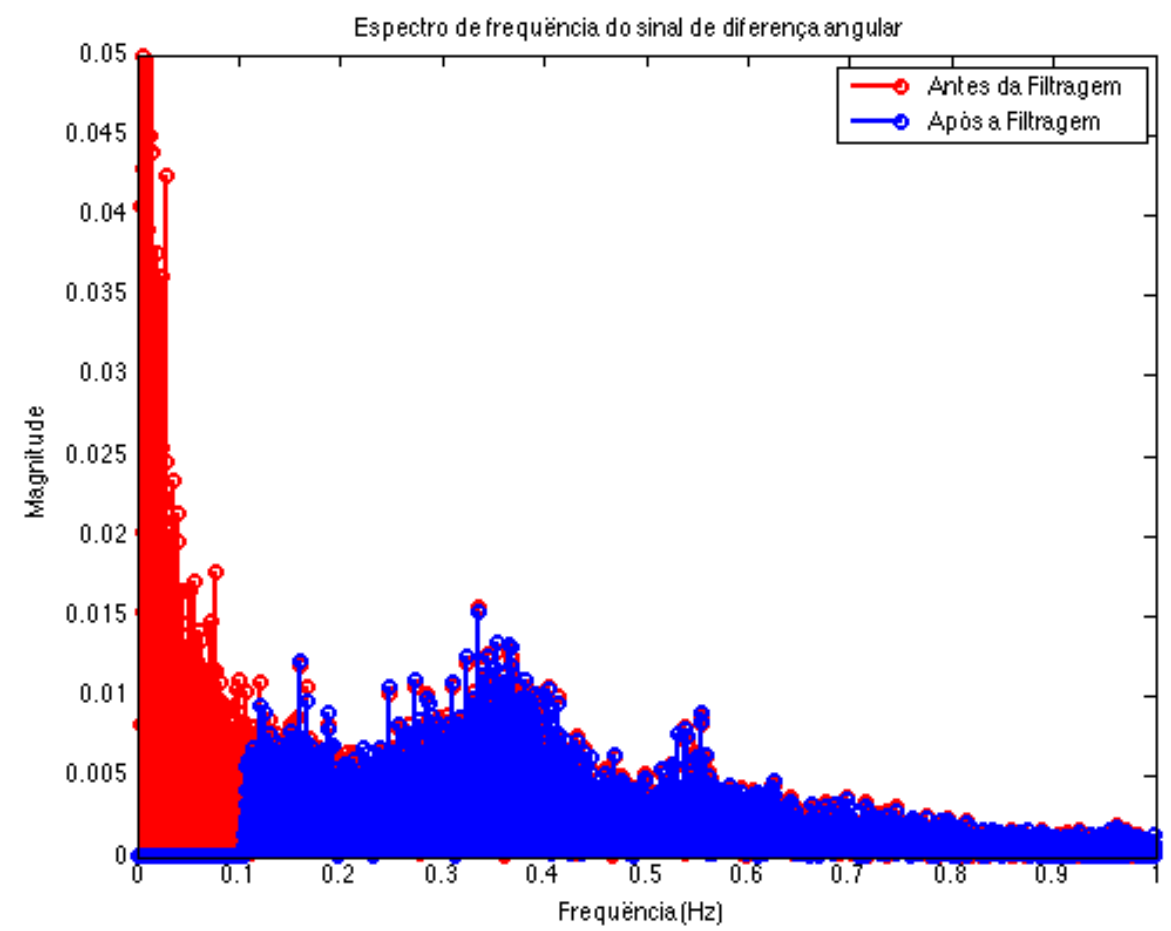

Figura 3.19: Espectro de frequências do sinal de diferença angular de tensão antes e após a filtragem

Já na década de 1960, Rudolph Kalman apresentou o FK [70], que utiliza técnicas de espaço de estados e formulações no domínio do tempo para a estimação linear recursiva ótima de estados[68]. O FK é utilizado para estimar estados de sistemas na presença de ruídos, desde que estejam disponíveis o modelo dinâmico do sistema, as covariâncias 
dos ruídos presentes no modelo e nas medições. Com isso é possível fazer a predição de estados desses sistemas, os quais são corrigidos a cada nova amostra obtida do sinal de saída ruidoso. O FK promove a propagação da densidade de probabilidade condicional dos estados de um sistema quando este pode ser descrito por um modelo linear e os ruídos de processo e de medição são brancos e Gaussianos [71]. O resultado é um estimador linear de estados ótimo estatisticamente em relação a qualquer função quadrada de estimação de erros [53, 69, 72]. Em sistemas dinâmicos, nem sempre é possível ou desejável medir todas as variáveis que se deseja controlar [69], e o FK é uma maneira de estimar estas variáveis por meio de medidas indiretas e ruidosas.

Apesar de a nomenclatura "filtro" normalmente remeter a dispositivos eletrônicos como indutores e capacitores que filtram sinais, o FK é, na verdade, um algoritmo de processamento de dados recursivo, que trabalha com sinais amostrados em tempo discreto, ao invés de sinais em tempo contínuo [71].

A técnica de mínimos quadrados para a estimação linear utiliza dados de medição $z$, os quais estão relacionados linearmente aos estados desconhecidos $x$ e ao ruído de medição $v$ pela seguinte expressão:

$$
z=H x+v
$$

O objetivo é estimar o vetor de estados $x$, de maneira que a soma do quadrado da diferença dos elementos de $z-H \hat{x}$ seja minimizada, sendo $\hat{x}$ o vetor de estados estimados, e $H$ uma matriz cujos parâmetros são conhecidos. Assim, deve-se minimizar a função de custo $J$, onde:

$$
J=(z-H \hat{x})^{T}(z-H \hat{x})
$$

Uma maneira de minimizar esta função custo é igualando sua derivada a zero.

$$
\frac{\partial J}{\partial \hat{x}}=0
$$

Procedendo desta maneira, tem-se a seguinte expressão para estimação do vetor de estados:

$$
\hat{x}=\left(H^{T} H\right)^{-1} H^{T} z
$$


sendo que a matriz $H^{T} H$ é chamada de matriz Graminiana. Se a matriz Graminiana for não-singular, ou seja, se o determinante for diferente de zero, o método dos mínimos quadrados determina os valores únicos das variáveis desconhecidas tal que o quadrado do desvio em relação às equações de restrição é minimizado. Se a matriz Graminiana for singular, então as variáveis desconhecidas serão não-observáveis [69].

De maneira simplista, o FK pode ser considerado como uma solução recursiva na forma de espaço de estados para os problemas de mínimos quadrados explorado por Gauss [73]. Diferente do método de Gauss, o FK não precisa de um bloco de dados, mas pode analisá-los de modo sequencial. [68].

Para exemplificar o funcionamento de um algoritmo de estimação de estados como o FK, apresenta-se a seguir um exemplo contido em [71] e em [68] que ilustra bem o comportamento do filtro.

Imagine que você está perdido no mar à noite e não tem idéia da sua localização. A única possibilidade de estimar sua localização é através do brilho das estrelas e da lua. A partir disso você estima sua localização em $t_{1}$ como sendo $z_{1}$, em apenas uma dimensão para simplificação do exemplo. Considerando a incerteza envolvida na estimação da sua localização por meio das estrelas você decide que a precisão dessa medição é tal que o desvio padrão é $\sigma_{z 1}$ ou, de modo equivalente, que a variância é $\sigma_{z 1}^{2}$.

Assim é possível estabelecer a densidade de probabilidade de $x\left(t_{1}\right)$, que é sua localização em $t_{1}$, condicionada à localização estimada $z_{1}$, conforme ilustrado na Figura 3.20.

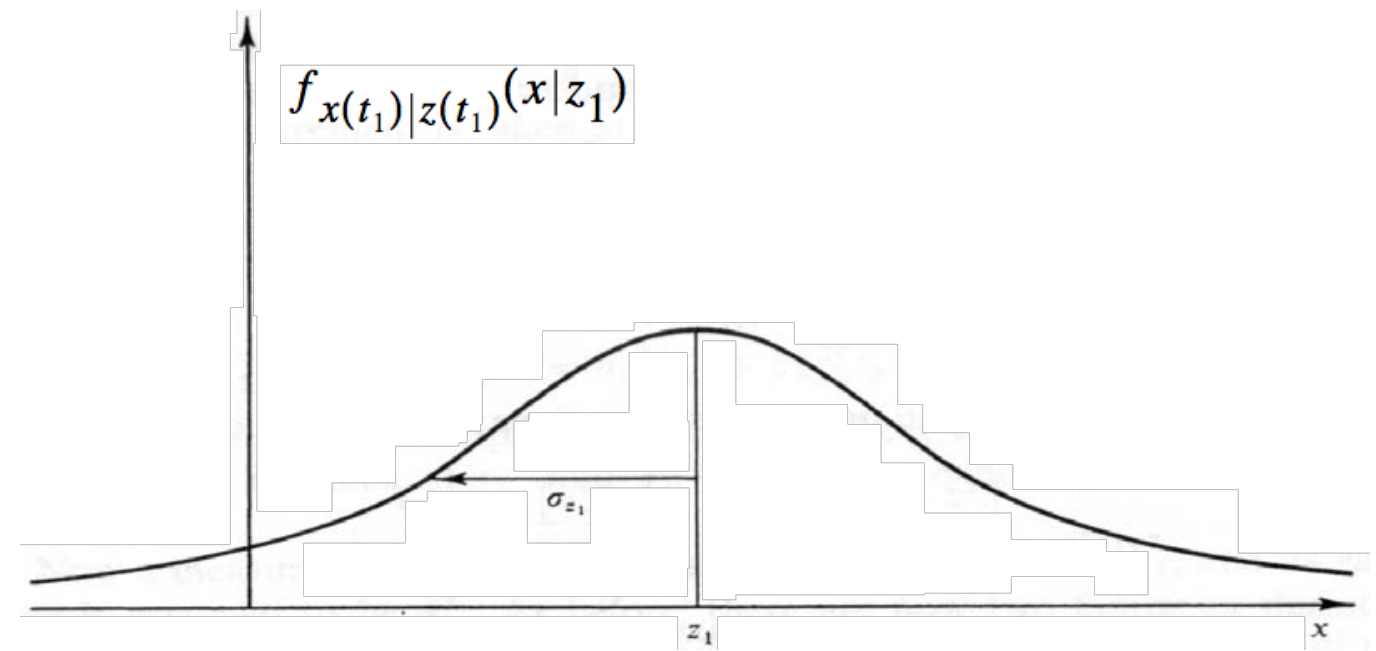

Figura 3.20: Densidade de probabilidade da localização baseada na estimativa $z_{1}[71]$ 
Essa figura ilustra a função de densidade de probabilidade $f_{x\left(t_{1}\right) \mid z\left(t_{1}\right)}\left(x \mid z_{1}\right)$ em função da localização estimada. Ela expressa a probabilidade de se estar em alguma localização baseada nas estimativas. Nota-se que $\sigma_{z 1}$ é uma medida direta da incerteza quanto a localização. Quanto maior $\sigma_{z 1}$, mais espalhado será o gráfico de probabilidade ao longo do eixo $x$. Para uma densidade Gaussiana, a probabilidade de a localização estar dentro dos limites definidos por $\sigma_{z 1}$, para cada lado da média, é de $68,3 \%$. A função de densidade de probabilidade Gaussiana, para uma variável com média $\mu$ e desvio padrão $\sigma$, é da seguinte forma:

$$
p(x)=\frac{1}{\sqrt{2 \pi \sigma^{2}}} e^{-\frac{(x-\mu)^{2}}{2 \sigma^{2}}}
$$

Assim, baseado na função de densidade de probabilidade, a melhor estimativa da posição em $t_{1}$ é $\hat{x}\left(t_{1}\right)=z_{1}$, e a variância do erro dessa estimativa é $\sigma_{x}^{2}\left(t_{1}\right)=\sigma_{z 1}^{2}$.

Imagine agora que em $t_{2}$, imediatamente após $t_{1}$, de tal forma que a posição real seja a mesma, um navegador experiente lhe diz que sua posição é $z_{2}$ com variância $\sigma_{z 2}^{2}$. Devido às habilidades do navegador, a variância $\sigma_{z 2}^{2}$ é menor que $\sigma_{z 1}^{2}$. A Figura 3.21 ilustra a densidade de probabilidade da localização considerando a estimativa $z_{2}$.

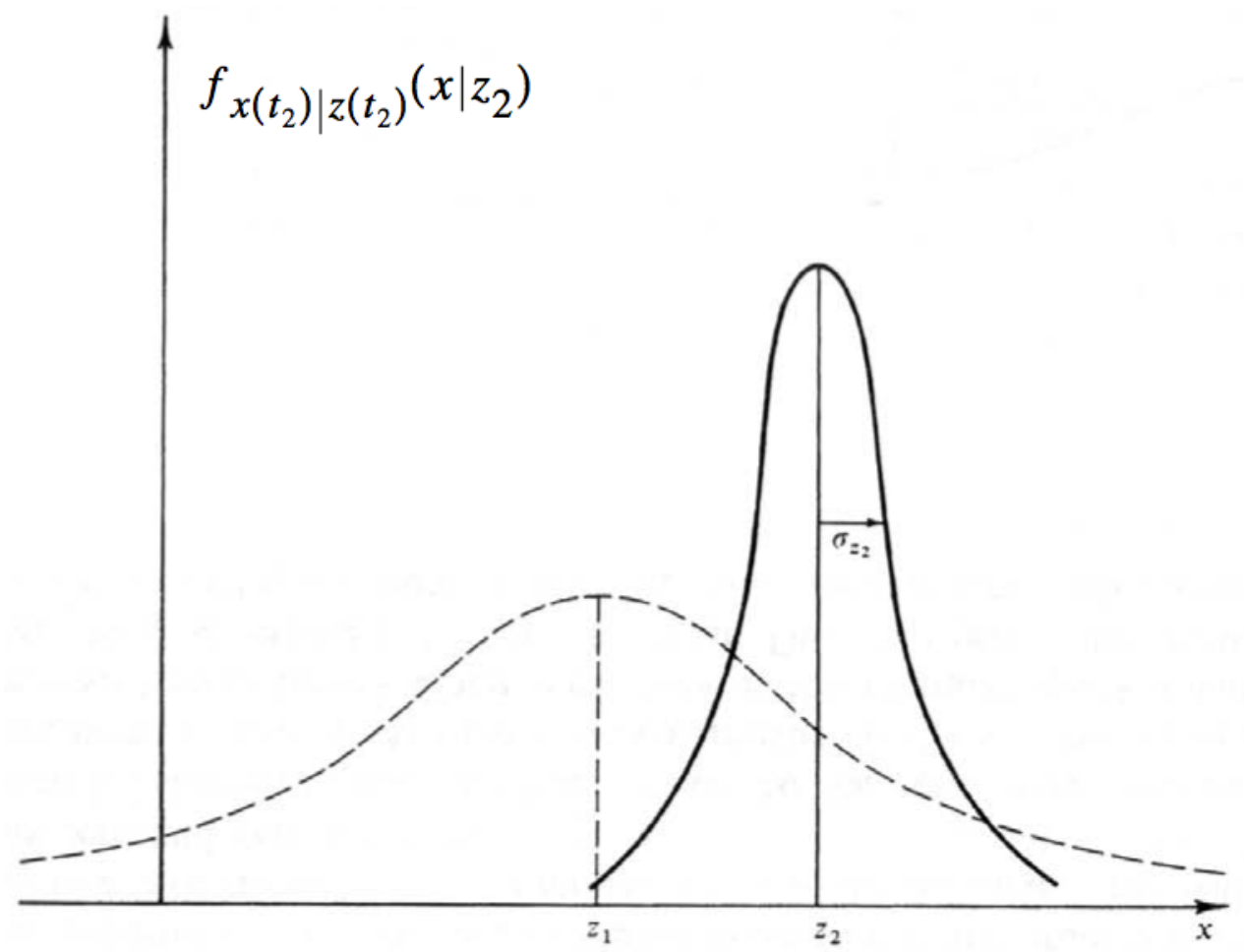

Figura 3.21: Densidade de probabilidade da localização baseada na estimativa $z_{2}[71]$

Observe que o pico da densidade de probabilidade baseada em $z_{2}$ é mais estreito, indi- 
cando uma confiança maior quanto a essa estimativa. Para obter uma estimativa mais precisa, é necessário combinar as informações contidas em $z_{1}$ e $z_{2}$. A nova estimativa de localização em $t_{2} \cong t_{1}$, dadas as estimativas $z_{1}$ e $z_{2}$, terá uma densidade de probabilidade Gaussiana com média $\mu$ e variância $\sigma^{2}$, ilustrada na Figura 3.22, dadas por (3.11) e (3.12).

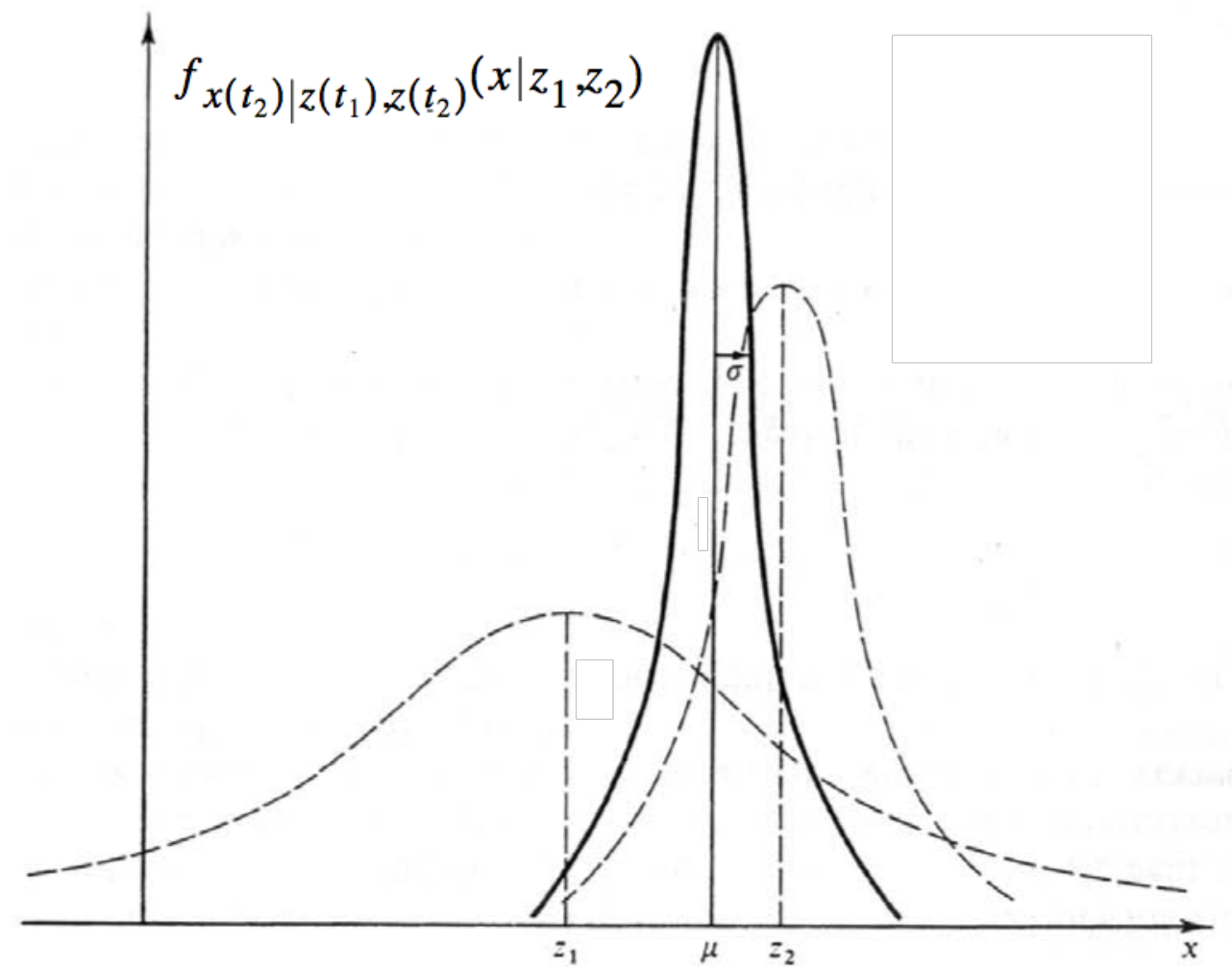

Figura 3.22: Densidade de probabilidade da localização baseada nas estimativas $z_{1}$ e $z_{2}$ [71]

$$
\begin{gathered}
\mu=\left[\frac{\sigma_{z 2}^{2}}{\left(\sigma_{z 1}^{2}+\sigma_{z 2}^{2}\right)}\right] z_{1}+\left[\frac{\sigma_{z 1}^{2}}{\left(\sigma_{z 1}^{2}+\sigma_{z 2}^{2}\right)}\right] z_{2} \\
\frac{1}{\sigma^{2}}=\left(\frac{1}{\sigma_{z 1}^{2}}\right)+\left(\frac{1}{\sigma_{z 2}^{2}}\right)
\end{gathered}
$$

Conforme (3.12), o valor de $\sigma^{2}$ é menor do que $\sigma_{z 1}^{2}$ ou $\sigma_{z 2}^{2}$, ou seja, a combinação das duas estimativas diminuiu a incerteza que havia quanto a estimativa da localização. Assim, a melhor estimativa de localização em $t_{2}$ é $\hat{x}\left(t_{2}\right)=\mu$, com uma variância associada de $\sigma^{2}$. A equação para $\hat{x}\left(t_{2}\right)$ pode ser reescrita como:

$$
\begin{aligned}
\hat{x}\left(t_{2}\right) & =\left[\frac{\sigma_{z 2}^{2}}{\left(\sigma_{z 1}^{2}+\sigma_{z 2}^{2}\right)}\right] z_{1}+\left[\frac{\sigma_{z 1}^{2}}{\left(\sigma_{z 1}^{2}+\sigma_{z 2}^{2}\right)}\right] z_{2} \\
& =z_{1}+\left[\frac{\sigma_{z 1}^{2}}{\left(\sigma_{z 1}^{2}+\sigma_{z 2}^{2}\right)}\right]\left[z_{2}-z_{1}\right]
\end{aligned}
$$


Adotando a forma utilizada no FK, que é descrita mais a frente, e considerando que $\hat{x}\left(t_{1}\right)=z_{1}$, a equação (3.13) pode ser reescrita como:

$$
\hat{x}\left(t_{2}\right)=\hat{x}\left(t_{1}\right)+K\left(t_{2}\right)\left[z_{2}-\hat{x}\left(t_{1}\right)\right]
$$

onde:

$$
K\left(t_{2}\right)=\frac{\sigma_{z 1}^{2}}{\left(\sigma_{z 1}^{2}+\sigma_{z 2}^{2}\right)}
$$

A equação (3.14) ilustra que a melhor estimativa que se tem em $t_{2}, \hat{x}\left(t_{2}\right)$, é igual a melhor estimativa anterior à estimativa $z_{2}, \hat{x}\left(t_{1}\right)$, mais uma ponderação ótima, $K\left(t_{2}\right)$, multiplicada pela diferença entre $z_{2}$ e $\hat{x}\left(t_{1}\right)$. Utilizando o valor de $K\left(t_{2}\right)$ obtido em (3.15) em (3.12), tem-se:

$$
\sigma_{x}^{2}\left(t_{2}\right)=\sigma_{x}^{2}\left(t_{1}\right)-K\left(t_{2}\right) \sigma_{x}^{2}\left(t_{1}\right)
$$

Propagando os valores de estimativa de localização e de variância ao longo do tempo, conforme (3.14) e (3.16), tem-se a melhor estimativa de localização a cada instante. Dessa forma, o objetivo do processo de estimação de estados, nesse caso a localização, é, a cada nova medição, ponderar as informações contidas na estimativa anterior e na nova medição para produzir uma nova estimativa dos estados, buscando sempre a minimização do erro das estimativas.

Observando a equação (3.15) conclui-se que quanto maior for a incerteza contida na medida $z_{2}$, menor será a confiança nesta medida e menor será o peso dado a ela na estimativa da localização em (3.14). Caso contrário, quanto menor for a variância $\sigma_{z 2}^{2}$, mais próximo o $K\left(t_{2}\right)$ será de 1 e o valor de $\hat{x}\left(t_{2}\right)$ se aproximará de $z_{2}$.

A descrição deste exemplo serve para ilustrar uma das principais características do FK, que é a sua separação em duas etapas: predição e correção. Na etapa de predição os estados são previstos com base nas medidas e incertezas das medições anteriores. E na etapa de correção os estados previstos são atualizados com base na medida e na incerteza da nova medição. Por ser um algoritmo recursivo, a necessidade de guardar as medições passadas é eliminada. Todas as informações prévias estão contidas na estimativa atual [68]. 
A seguir, descreve-se o funcionamento e o equacionamento do FK. Existem na literatura diversas formas de derivação do FK, aqui é apresentada uma delas [68, 74]. Suponha um sistema de espaço de estados autônomo estocástico em tempo discreto dado pelas seguintes equações lineares:

$$
\begin{gathered}
x_{k+1}=A x_{k}+w_{k} \\
y_{k}=C x_{k}+v_{k}
\end{gathered}
$$

em que $x_{k} \in \mathbb{R}^{n}$ é o vetor de estados; $y_{k} \in \mathbb{R}^{q}$ é o vetor de saídas; $A \in \mathbb{R}^{n \times n}$ é a matriz de transição de estados; $C \in \mathbb{R}^{q \times n}$ é a matriz de saída; $w_{k} \in \mathbb{R}^{n}$ e $v_{k} \in \mathbb{R}^{q}$ são variáveis aleatórias que representam os ruídos de modelo e de medição, respectivamente. As variáveis aleatórias são caracterizadas como ruído branco Gaussiano, com média nula e descorrelacionadas, com covariâncias de modelo $Q \in \mathbb{R}^{n \times n}$, ou seja $p\left(w_{k}\right) \sim$ $N(0, Q)$ e de medição $R \in \mathbb{R}^{q \times q}, p\left(v_{k}\right) \sim N(0, R)$. As variáveis $w_{k}$ e $v_{k}$ também são descorrelacionadas entre si, de tal forma que:

$$
E\left[w_{k} v_{k}^{T}\right]=0
$$

onde $E[\bullet]$ é o operador valor esperado. O objetivo do FK é estimar os estados $x_{k}$ a cada passo $k$, com base na dinâmica do sistema e nas medições. O diagrama de blocos da Figura 3.23 ilustra o processo de estimação de estados.

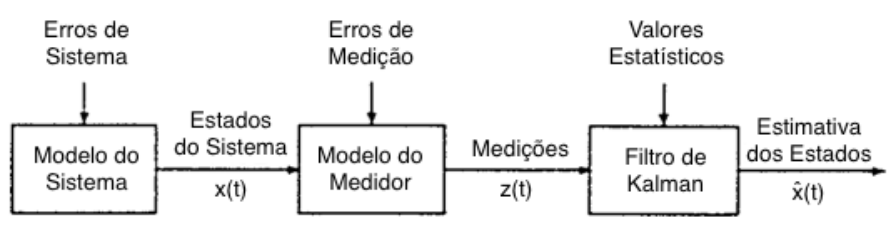

Figura 3.23: Diagrama de blocos de um modelo de estimador de estados [69]

Defini-se o vetor $\hat{x}_{k}^{-}$como sendo a estimativa a priori do vetor $x_{k}$ dadas todas as informações do processo anteriores ao passo $k$, e $\hat{x}_{k}$ como sendo a estimativa a posteriori do vetor $x_{k}$ dada a medida $y_{k}$ [72]. Assim podem ser definidas as estimativas de erro a priori e a posteriori como:

$$
\begin{gathered}
e_{k}^{-}=x_{k}-\hat{x}_{k}^{-} \\
e_{k}=x_{k}-\hat{x}_{k}
\end{gathered}
$$


E a covariância das estimativas de erro a priori e a posteriori são definidas por (3.22) e $(3.23)$.

$$
\begin{gathered}
P_{k}^{-}=E\left[e_{k}^{-} e_{k}^{-T}\right] \\
P_{k}=E\left[e_{k} e_{k}^{T}\right]
\end{gathered}
$$

Uma maneira de calcular a estimativa a posteriori do vetor de estados $x_{k}$ em função do seu valor a priori é utilizando a equação (3.14), da seguinte forma:

$$
\hat{x}_{k}=\hat{x}_{k}^{-}+K_{k}\left(y_{k}-C \hat{x}_{k}^{-}\right)
$$

No entanto, para chegar nesta formulação e explicar o equacionamento do FK, é preciso voltar um passo atrás e simplesmente definir que a estimativa do vetor de estados $x_{k}$ a posteriori será uma combinação linear da estimativa a priori de $x_{k}$ e do vetor de medições $y_{k}$, da seguinte forma:

$$
\hat{x}_{k}=K_{k}^{1} \hat{x}_{k}^{-}+K_{k} y_{k}
$$

As matrizes $K_{k}^{1}$ e $K_{k}$ são desconhecidas, e sua determinação parte do teorema da ortogonalidade [69]. Deste modo, tem-se o seguinte:

$$
\begin{aligned}
& E\left[e_{k} y_{i}^{T}\right]=0 \quad \text { para } \quad i=0,1, \ldots, k-1 \\
& E\left[e_{k}^{-} y_{i}^{T}\right]=0 \quad \text { para } \quad i=0,1, \ldots, k-1
\end{aligned}
$$

Substituindo (3.18), (3.25) e (3.21) em (3.26):

$$
\begin{aligned}
E\left[\left(x_{k}-\left(K_{k}^{1} \hat{x}_{k}^{-}+K_{k}\left(C x_{k}+v_{k}\right)\right)\right) y_{i}^{T}\right] & =0 \\
E\left[\left(x_{k}-K_{k}^{1} \hat{x}_{k}^{-}-K_{k}\left(C x_{k}+v_{k}\right)\right) y_{i}^{T}\right] & =0 \quad \text { para } i=0,1, \ldots, k-1 \\
E\left[\left(x_{k}-K_{k}^{1} \hat{x}_{k}^{-}-K_{k} C x_{k}-K_{k} v_{k}\right) y_{i}^{T}\right] & =0
\end{aligned}
$$

Considerando que $v_{k}$ e $y_{i}$ são descorrelacionados, $E\left[v_{k} y_{i}^{T}\right]=0$, e substituindo (3.20) e (3.27) em (3.28), a equação (3.28) pode ser reescrita da seguinte forma: 


$$
\begin{aligned}
E\left[x_{k} y_{i}^{T}-K_{k}^{1} \hat{x}_{k}^{-} y_{i}^{T}-K_{k} C x_{k} y_{i}^{T}-K_{k} v_{k} y_{i}^{T}\right] & =0 \\
E\left[x_{k} y_{i}^{T}-K_{k}^{1} \hat{x}_{k}^{-} y_{i}^{T}-K_{k} C x_{k} y_{i}^{T}\right] & =0 \\
E\left[x_{k} y_{i}^{T}-K_{k}^{1} \hat{x}_{k}^{-} y_{i}^{T}-K_{k} C x_{k} y_{i}^{T}\right] & =0 \\
E\left[\left(I-K_{k} C\right) x_{k} y_{i}^{T}-K_{k}^{1} \hat{x}_{k}^{-} y_{i}^{T}\right] & =0 \quad \text { para } \quad i=0,1, \ldots, k-1 \\
E\left[\left(I-K_{k} C\right) x_{k} y_{i}^{T}-K_{k}^{1} \hat{x}_{k}^{-} y_{i}^{T}+K_{k}^{1} x_{k} y_{i}^{T}-K_{k}^{1} x_{k} y_{i}^{T}\right] & =0 \\
E\left[\left(I-K_{k} C-K_{k}^{1}\right) x_{k} y_{i}^{T}+K_{k}^{1}\left(x_{k}-\hat{x}_{k}^{-}\right) y_{i}^{T}\right] & =0 \\
\left(I-K_{k} C-K_{k}^{1}\right) E\left[x_{k} y_{i}^{T}\right] & =0
\end{aligned}
$$

Logo, a solução para (3.29) é:

$$
\begin{gathered}
\left(I-K_{k} C-K_{k}^{1}\right)=0 \\
K_{k}^{1}=I-K_{k} C
\end{gathered}
$$

Substituindo (3.31) em (3.25):

$$
\hat{x}_{k}=\hat{x}_{k}^{-}+K_{k}\left(y_{k}-C \hat{x}_{k}^{-}\right)
$$

Essa equação (3.32) é igual a equação (3.24) da hipótese inicial.

A diferença $\left(y_{k}-C x_{k}^{-}\right)$é chamada de inovação ou resíduo. Ela mostra qual a diferença entre o valor estimado e o valor medido de $y_{k}$.

A matriz $K_{k} \in \mathbb{R}^{n \times m}$ é chamada de Matriz Ganho de Kalman, e é definida de tal maneira que a covariância do erro a posteriori (3.23) seja minimizada. Para encontrar esta matriz, remete-se novamente ao princípio da ortogonalidade. A partir dele, tem-se que:

$$
\begin{gathered}
E\left[e_{k} y_{k}^{T}\right]=0 \quad \mathrm{e} \\
E\left[e_{k} \hat{y}_{k}^{T}\right]=0
\end{gathered}
$$

onde $\hat{y}_{k}$ é o vetor de estimativa da medida $y_{k}$, tendo em vista os valores das medições $y_{1}, y_{2}, \ldots, y_{k-1}$. Define-se então o erro na estimativa da medida $y_{k}$ : 


$$
\begin{aligned}
e_{y} & =y_{k}-\hat{y}_{k} \\
& =y_{k}-C \hat{x}_{k}^{-} \\
& =C x_{k}+v_{k}-C \hat{x}_{k}^{-} \\
& =C\left(x_{k}-\hat{x}_{k}^{-}\right)+v_{k} \\
& =C\left(e_{k}^{-}\right)+v_{k}
\end{aligned}
$$

Subtraindo (3.34) de (3.33) e substituindo (3.35):

$$
\begin{aligned}
E\left[e_{k} y_{k}^{T}-e_{k} \hat{y}_{k}^{T}\right] & =0 \\
E\left[e_{k}\left(y_{k}^{T}-\hat{y}_{k}^{T}\right)\right] & =0 \\
E\left[e_{k} e_{y}^{T}\right] & =0
\end{aligned}
$$

Substituindo (3.21), (3.35) e (3.25) em (3.36)

$$
\begin{aligned}
E\left[e_{k} e_{y}^{T}\right] & =0 \\
E\left[\left(x_{k}-\hat{x}_{k}\right)\left(y_{k}-C \hat{x}_{k}^{-}\right)^{T}\right] & =0 \\
E\left[\left(x_{k}-K_{k}^{1} \hat{x}_{k}^{-}-K_{k} y_{k}\right)\left(y_{k}-C \hat{x}_{k}^{-}\right)^{T}\right] & =0
\end{aligned}
$$

Agora substituindo (3.31), (3.20) e (3.18) em (3.37):

$$
\begin{aligned}
E\left[\left(x_{k}-K_{k}^{1} \hat{x}_{k}^{-}-K_{k} y_{k}\right)\left(y_{k}-C \hat{x}_{k}^{-}\right)^{T}\right] & =0 \\
E\left[\left(x_{k}-\left(I-K_{k} C\right) \hat{x}_{k}^{-}-K_{k}\left(C x_{k}+v_{k}\right)\right)\left(\left(C x_{k}+v_{k}\right)-C \hat{x}_{k}^{-}\right)^{T}\right] & =0 \\
E\left[\left(\left(x_{k}-\hat{x}_{k}^{-}\right)+K_{k} C \hat{x}_{k}^{-}-K_{k}\left(C x_{k}+v_{k}\right)\right)\left(C\left(x_{k}-\hat{x}_{k}^{-}\right)+v_{k}\right)^{T}\right] & =0 \\
E\left[\left(\left(x_{k}-\hat{x}_{k}^{-}\right)-K_{k} C\left(x_{k}-\hat{x}_{k}^{-}\right)-K_{k} v_{k}\right)\left(C\left(x_{k}-\hat{x}_{k}^{-}\right)+v_{k}\right)^{T}\right] & =0 \\
E\left[\left(e_{k}^{-}-K_{k} C e_{k}^{-}-K_{k} v_{k}\right)\left(C e_{k}^{-}+v_{k}\right)^{T}\right] & =0
\end{aligned}
$$

Por definição, a covariância do erro a priori é (3.22). Desta forma, a equação (3.38) pode ser reescrita assim:

$$
\left[I-K_{k} C\right] P_{k}^{-} C^{T}-K_{k} R=0
$$

E assim a matriz Ganho de Kalman é definida em função da covariância do erro a priori $\left(P_{k}^{-}\right)$da seguinte forma:

$$
K_{k}=P_{k}^{-} C^{T}\left[C P_{k}^{-} C^{T}+R\right]^{-1}
$$


Resta agora apenas demonstrar como ocorre a propagação da estimativa de covariância do erro dos estados. Para isso deduz-se as equações que definem a estimativa de covariância do erro a priori $\left(P_{k}^{-}\right)$e a posteriori $\left(P_{k}\right)$.

Para obter $P_{k}$, primeiro subtrai-se $x_{k}$ de ambos os lados da equação (3.32) e substitui-se a equação (3.18):

$$
\begin{aligned}
\hat{x}_{k}-x_{k} & =\hat{x}_{k}^{-}+K_{k}\left(C x_{k}+v_{k}-C \hat{x}_{k}^{-}\right)-x_{k} \\
e_{k} & =e_{k}^{-}+K_{k}\left(C x_{k}+v_{k}-C \hat{x}_{k}^{-}\right) \\
e_{k} & =e_{k}^{-}+K_{k} C x_{k}+K_{k} v_{k}-K_{k} C \hat{x}_{k}^{-} \\
e_{k} & =e_{k}^{-}-K_{k} C e_{k}^{-}+K_{k} v_{k} \\
e_{k} & =\left(I-K_{k} C\right) e_{k}^{-}+K_{k} v_{k}
\end{aligned}
$$

Substituindo (3.41) em (3.23) e considerando que $E\left[e_{k}^{-} v_{k}^{T}\right]=0$, obtém-se o seguinte:

$$
\begin{aligned}
P_{k} & =E\left[\left(\left(I-K_{k} C\right) e_{k}^{-}+K_{k} v_{k}\right)\left(\left(I-K_{k} C\right) e_{k}^{-}+K_{k} v_{k}\right)^{T}\right] \\
& =E\left[\left(\left(I-K_{k} C\right) e_{k}^{-} e_{k}^{-T}\left(I-K_{k} C\right)^{T}+K_{k} v_{k} v_{k}^{T} K_{k}^{T}\right]\right. \\
& =\left(I-K_{k} C\right) P_{k}^{-}\left(I-K_{k} C\right)^{T}+K_{k} R K_{k}^{T}
\end{aligned}
$$

Resta apenas encontrar a matriz de covariâncias de erro a priori das estimativas dos estados, $P_{k}^{-}$. Para tanto, primeiro subtrai-se $x_{k}$ de ambos os lados da equação de propagação dos estados (3.17). Deve ser considerado um sistema autônomo e que os vetores dos estados são estimativas, da seguinte forma:

$$
\begin{aligned}
\hat{x}_{k}^{-} & =A \hat{x}_{k-1} \\
\hat{x}_{k}^{-}-x_{k} & =A \hat{x}_{k-1}-x_{k} \\
e_{k}^{-} & =A\left(\hat{x}_{k-1}-x_{k-1}\right)-w_{k-1} \\
& =A e_{k-1}-w_{k-1}
\end{aligned}
$$

Depois basta substituir (3.43) em (3.22). Considerando que $E\left[e_{k-1} w_{k-1}^{T}\right]=0$, obtém-se:

$$
\begin{aligned}
& P_{k}^{-}=E\left[\left(A e_{k-1}-w_{k-1}\right)\left(A e_{k-1}-w_{k-1}\right)^{T}\right] \\
& P_{k}^{-}=A E\left[e_{k-1} e_{k-1}^{T}\right] A^{T}+E\left[w_{k-1} w_{k-1}^{T}\right] \\
& P_{k}^{-}=A P_{k-1} A^{T}+Q
\end{aligned}
$$


Por fim, estão definidas todas as equações necessárias para o algoritmo de estimação de estados do FK. A cada passo $k$, o FK primeiramente estima o valor mais provável de $x_{k}$ baseado nos valores passados dos estados até $x_{k-1}$. Posteriormente, esta estimação é atualizada como uma combinação linear entre a estimação obtida anteriormente e a diferença entre a medição atual e a medida estimada, conforme equação (3.32). Como os ruídos de processo e de medição possuem distribuição Gaussiana, a estimativa dos estados também terá distribuição Gaussiana.

Para iniciar a estimação de estados por meio do FK é preciso ter uma estimativa para $\hat{x}_{0}$ e para $P_{0}$. O valor $\hat{x}_{0}$ é uma estimativa inicial do vetor de estados $x_{0}$, e $P_{0}$ é a matriz de covariâncias do erro de $\hat{x}_{0}$ em relação aos valores reais do vetor de estados $x_{0}$. Quanto menor os valores de covariância da matriz $P_{0}$, maior é a confiança que se tem nos valores iniciais do vetor de estados $\hat{x}_{0}$.

\subsubsection{Algoritmo do Filtro de Kalman}

O algoritmo de estimação de estados do FK é composto por cinco equações divididas em duas etapas [72]. Na primeira, chamada de predição, é feita uma estimação dos estados do sistema para a amostra $k$ com base nos valores dos estados obtidos até a amostra $k-1$. Na segunda etapa, chamada de correção, os valores dos estados estimados na etapa anterior são corrigidos com base na medida de inovação.

As equações que descrevem a etapa de predição do algoritmo do FK são as seguintes:

$$
\begin{gathered}
\hat{x}_{k}^{-}=A \hat{x}_{k-1} \\
P_{k}^{-}=A P_{k-1} A^{T}+Q
\end{gathered}
$$

E a etapa de correção é descrita pelas seguintes equações:

$$
\begin{gathered}
K_{k}=P_{k}^{-} C^{T}\left(C P_{k}^{-} C^{T}+R\right)^{-1} \\
\hat{x}_{k}=\hat{x}_{k}^{-}+K_{k}\left(y_{k}-C \hat{x}_{k}^{-}\right) \\
P_{k}=\left(I-K_{k} C\right) P_{k}^{-}\left(I-K_{k} C\right)^{T}+K_{k} R K_{k}^{T}
\end{gathered}
$$

Na etapa de correção é calculado o ganho de Kalman $K_{k}$, os estados $\hat{x}_{k}^{-}$são corrigidos com base na inovação e a matriz de covariâncias de erros das estimativas $P_{k}$ é atualizada. Considerando que os ruídos no processo e nas medições tem probabilidade de 
distribuição normal com covariâncias $Q$ e $R$, a matriz $P_{k}$ estima, a cada passo, qual será a covariância do erro da estimativa do passo seguinte.

\subsubsection{Algoritmo do Filtro de Kalman Estendido}

O equacionamento apresentado na Subseção 3.4.1 sobre o FK é válido para situações em que as matrizes A e C são conhecidas e representam modelos lineares. Casos mais gerais requerem uma abordagem não linear. Um modelo de um sistema não linear autônomo pode ser descrito por:

$$
\begin{gathered}
x_{k}=f\left(x_{k-1}, w_{k-1}\right) \\
y_{k}=h\left(x_{k-1}, v_{k-1}\right)
\end{gathered}
$$

Algoritmos de estimação para sistemas não lineares, normalmente não podem ser expressos de forma fechada, e requerem algum método de aproximação [68]. Modificações foram introduzidas no algoritmo do FK [72], ficando a nova denominação conhecida como FKE [69].

O FKE realiza linearizações a cada passo por meio de expansões da série de Taylor de primeira ordem, de forma que as matrizes do sistema de espaço de estados no FK são substituídas no passo $k$ pelas linearizações no FKE. Assim, as equações do FKE na etapa de predição são as seguintes:

$$
\begin{gathered}
\hat{x}_{k}^{-}=f\left(\hat{x}_{k-1}\right) \\
P_{k}^{-}=A_{k-1} P_{k-1} A_{k-1}^{T}+Q
\end{gathered}
$$

E na etapa de correção:

$$
\begin{gathered}
K_{k}=P_{k}^{-} C_{k-1}^{T}\left(C_{k-1} P_{k}^{-} C_{k-1}^{T}+R\right)^{-1} \\
\hat{x}_{k}=\hat{x}_{k}^{-}+K_{k}\left(y_{k}-h\left(\hat{x}_{k}^{-}\right)\right) \\
P_{k}=\left(I-K_{k} C_{k-1}\right) P_{k}^{-}\left(I-K_{k} C_{k-1}\right)^{T}+K_{k} R K_{k}^{T}
\end{gathered}
$$

sendo que as matrizes $A_{k}$ e $C_{k}$ são as matrizes Jacobianas de $f\left(\hat{x}_{k}\right)$ e $h\left(\hat{x}_{k}\right)$, respectivamente. Essas matrizes precisam ser calculadas a cada amostra $k$, uma vez que 
dependem dos valores dos estados estimados. Uma das diferenças entre o FKE e o FK convencional é que no primeiro caso o ganho $K_{k}$ irá depender do vetor de estados estimado, o qual depende das matrizes de transição de estados e de medição $\left(A_{k}\right.$ e $\left.C_{k}\right)$. Isso acontece porque escolheu-se linearizar $A_{k}$ e $C_{k}$ em relação aos valores estimados dos estados. Assim, a matriz $K_{k}$ deve ser calculada a cada passo $k$, não podendo ser calculada e armazenada previamente à coleta de medições [68].

\subsubsection{Identificação de parâmetros na forma de estados}

Nesta seção introduz-se o problema de identificação de parâmetros de um modelo de sistema na forma de um sistema de espaço de estados. Em todas as descrições das seções anteriores quanto ao FK, tanto o convencional quanto o FKE, pressupõe-se o conhecimento prévio de todos os parâmetros do sistema que compõe as matrizes $A$ e $C$.

No entanto, há situações em que alguns parâmetros não são conhecidos e existe a necessidade de identificá-los. Nestes casos, o problema de estimação de estados torna-se um problema de estimação não linear [69]. Uma das maneiras de promover a identificação dos parâmetros é por meio da aplicação do FKE [68].

A ideia é simular o parâmetro na forma de um estado e admiti-lo invariante em um horizonte de tempo suposto como regime permanente. Ou seja, no tempo contínuo, se $\theta(t)$ é um vetor de estados representando parâmetros, então em regime permanente $\dot{\theta}(t)=0$. Em tempo discreto, esta expressão pode ser aproximada por $\theta_{k+1} \approx \theta_{k}$. Evidentemente, a concepção de "regime permanente" é relativa, pois o sistema é dinâmico, está constantemente sujeito a perturbações na forma de ruído ou mesmo distúrbios de outra natureza [75]. Por isso, admite-se que o estado de regime seja alcançado ao final de uma janela finita de dados, definida em função do problema. Em geral, ao se lidar com sinais amostrados de PMU, uma janela de 10 minutos é adequada para se estimar um conjunto de parâmetros admitidos invariantes [44].

Um sistema de espaço de estados em tempo discreto dado pelas equações (3.17) e (3.18), em que existam parâmetros a serem identificados pode ser modelado da seguinte forma:

$$
\begin{gathered}
x_{k+1}=A\left(\theta_{k}\right) x_{k}+w_{k} \\
y_{k}=C\left(\theta_{k}\right) x_{k}+v_{k} \\
\theta_{k+1}=\theta_{k}+w_{p}
\end{gathered}
$$


Considerando que os parâmetros são modelados como estados, o novo vetor de estados aumentado terá a seguinte forma:

$$
z_{k}=\left[\begin{array}{l}
x_{k} \\
\theta_{k}
\end{array}\right]
$$

E o novo sistema de espaço de estados com o vetor de estados aumentado pode ser escrito da seguinte maneira:

$$
\begin{gathered}
z_{k+1}=\left[\begin{array}{cc}
A\left(\theta_{k}\right) & 0 \\
0 & I
\end{array}\right] z_{k}+\left[\begin{array}{l}
w_{k} \\
w_{p}
\end{array}\right] \\
y_{k}=\left[\begin{array}{ll}
C\left(\theta_{k}\right) & 0
\end{array}\right] z_{k}+v_{k}
\end{gathered}
$$

Considerando que os parâmetros a serem identificados são invariantes no tempo, o sistema de espaço de estados descrito desta forma continua linear, pois as novas matrizes de transição de estados e de medição são constantes [68].

No entanto, ao modelar o sistema desta maneira com o objetivo de aplicar o FK, essas novas matrizes de transição de estados e de medição serão dependentes de estados a serem estimados por meio do próprio filtro. Durante o processo de estimação, o vetor de estados varia conforme o desenvolvimento do algoritmo e pelo mesmo motivo irão variar também as matrizes de transição de estados e de medição. Assim, sob o ponto de vista de estimação de estados, o sistema de espaço de estados para identificação de parâmetros será não linear. Por esse motivo utiliza-se o FKE nos processos de identificação de parâmetros de sistemas, sendo necessário calcular as matrizes Jacobianas a cada passo do algoritmo.

\subsection{Modelagem do Sistema}

O objetivo deste trabalho é a identificação de modos dominantes de um sistema elétrico do qual não se conhece o modelo e não é possível obter uma modelagem precisa em tempo real, inclusive porque os parâmetros do sistema elétrico são variantes no tempo. Além disso, os sinais utilizados na identificação são dados de ambiente provenientes de PMUs instaladas no sistema elétrico e sujeitos apenas a distúrbios do tipo ruído ambiente. 
Um sistema que tenha um único modo complexo possui a equação característica da seguinte forma:

$$
s^{2}+b_{1} s+a_{1}=0
$$

onde $a_{1}$ e $b_{1}$ são parâmetros do sistema. Uma representação na forma de espaço de estados em tempo contínuo de um sistema autônomo estocástico, com ruído de modelo $w$ e de medição $v$, com polos iguais aos da equação característica (3.63), pode ser expressa da seguinte maneira, chamada de forma canônica observável de um sistema linear de espaço de estados [76]:

$$
\begin{aligned}
{\left[\begin{array}{l}
\dot{x}_{1} \\
\dot{x}_{2}
\end{array}\right] } & =\left[\begin{array}{ll}
0 & -a_{1} \\
1 & -b_{1}
\end{array}\right]\left[\begin{array}{l}
x_{1} \\
x_{2}
\end{array}\right]+w \\
y & =\left[\begin{array}{ll}
0 & 1
\end{array}\right]\left[\begin{array}{l}
x_{1} \\
x_{2}
\end{array}\right]+v
\end{aligned}
$$

em que os estados são $x_{1}$ e $x_{2}$, os parâmetros são $a_{1}$ e $b_{1}$, e $y$ é o vetor de saída. A covariância associada ao ruído de modelo é $Q$ enquanto a de medição é $R$.

Com o intuito de evitar equívocos no entendimento desta dissertação, deste ponto em diante do texto é utilizada a notação $\bar{Q}$ e $\bar{R}$ para fazer referência às matrizes diagonais de covariâncias, e $Q$ e $R$ para referir aos elementos da diagonal dessas matrizes.

Os modos de oscilação deste sistema, ou polos, equivalem aos autovalores $(\lambda)$ da matriz de transição de estados. Os autovalores podem ser determinados conforme equacionamento a seguir, a partir das raízes da equação característica desta matriz.

$$
\begin{aligned}
& \operatorname{det}|\lambda I-A|=0 \\
& \operatorname{det}\left|\left[\begin{array}{ll}
\lambda & 0 \\
0 & \lambda
\end{array}\right]-\left[\begin{array}{cc}
0 & -a_{1} \\
1 & -b_{1}
\end{array}\right]\right|=0 \\
& \operatorname{det}\left|\left[\begin{array}{cc}
\lambda & a_{1} \\
-1 & \lambda+b_{1}
\end{array}\right]\right|=0 \\
& \lambda\left(\lambda+b_{1}\right)-(-1) a_{1}=0 \\
& \lambda^{2}+b_{1} \lambda+a_{1}=0
\end{aligned}
$$




$$
\begin{aligned}
& \lambda=\frac{-b_{1} \pm \sqrt{b_{1}^{2}-4 a_{1}}}{2} \\
& \lambda=-\frac{b_{1}}{2} \pm \frac{\sqrt{b_{1}^{2}-4 a_{1}}}{2} \\
& \lambda=-\frac{b_{1}}{2} \pm \sqrt{\frac{b_{1}^{2}}{4}-a_{1}} \\
& \lambda=-\frac{b_{1}}{2} \pm j \sqrt{a_{1}-\left(\frac{b_{1}}{2}\right)^{2}}
\end{aligned}
$$

Assim, para um autovalor definido por $\lambda=\sigma \pm j \omega$, tem-se que:

$$
\begin{gathered}
\sigma=-\frac{b_{1}}{2} \\
\omega=\sqrt{a_{1}-\left(\frac{b_{1}}{2}\right)^{2}}
\end{gathered}
$$

Os parâmetros $a_{1}$ e $b_{1}$ também podem ser incluídos como estados do FKE, conforme descrito na seção 3.4.3, e assim estimados a cada iteração do algoritmo [3, 27, 53, 77]. Desta forma, o sistema (3.64)-(3.65) é não linear e tem duas equações e quatro variáveis de estado $\left(x_{1}, x_{2}, a_{1}\right.$ e $\left.b_{1}\right)$. As outras duas equações que formam o sistema de espaço de estados a serem estimados são justamente $\dot{a}_{1}=0$ e $\dot{b}_{1}=0$.

A transformação de sistema contínuo em sistema discreto é feita aproximando-se a derivada no tempo pelo método das diferenças finitas de Newton, da seguinte forma:

$$
\dot{x}=\frac{x_{k+1}-x_{k}}{h}
$$

onde $h$ é o período de amostragem. Consequentemente, tendo o sistema somente dois parâmetros, os quais representam um modo de oscilação, as equações de espaço de estados serão:

$$
\begin{aligned}
& {\left[\begin{array}{l}
x_{1, k+1} \\
x_{2, k+1}
\end{array}\right]=\left[\begin{array}{l}
x_{1, k} \\
x_{2, k}
\end{array}\right]+h\left[\begin{array}{cc}
0 & -a_{1} \\
1 & -b_{1}
\end{array}\right]\left[\begin{array}{l}
x_{1, k} \\
x_{2, k}
\end{array}\right]+h w} \\
& \left.\left[\begin{array}{l}
x_{1, k+1} \\
x_{2, k+1}
\end{array}\right]=\left[\begin{array}{ll}
1 & 0 \\
0 & 1
\end{array}\right]+\left[\begin{array}{ll}
0 & -h a_{1} \\
h & -h b_{1}
\end{array}\right]\right]\left[\begin{array}{l}
x_{1, k} \\
x_{2, k}
\end{array}\right]+h w \\
& {\left[\begin{array}{l}
x_{1, k+1} \\
x_{2, k+1}
\end{array}\right]=\left[\begin{array}{cc}
1 & -h a_{1} \\
h & 1-h b_{1}
\end{array}\right]\left[\begin{array}{l}
x_{1, k} \\
x_{2, k}
\end{array}\right]+w_{k}}
\end{aligned}
$$




$$
\left[\begin{array}{l}
a_{1, k+1} \\
b_{1, k+1}
\end{array}\right]=\left[\begin{array}{l}
a_{1, k} \\
b_{1, k}
\end{array}\right]+w_{p}
$$

em que $w_{p}$ é o ruído de modelagem do processo, mas agora associado aos parâmetros, com média nula e covariância $Q_{P}$. Já $w_{k}$ é o ruído associado aos estados propriamente ditos, com média nula e covariância $Q_{X}$. Em uma forma compacta, é o equivalente a se ter o sistema aumentado:

$$
\begin{gathered}
{\left[\begin{array}{l}
x_{1, k+1} \\
x_{2, k+1} \\
a_{1, k+1} \\
b_{1, k+1}
\end{array}\right]=\left[\begin{array}{cccc}
1 & -h a_{1, k} & 0 & 0 \\
h & 1-h b_{1, k} & 0 & 0 \\
0 & 0 & 1 & 0 \\
0 & 0 & 0 & 1
\end{array}\right]\left[\begin{array}{l}
x_{1, k} \\
x_{2, k} \\
a_{1, k} \\
b_{1, k}
\end{array}\right]+\left[\begin{array}{l}
w_{k} \\
w_{p}
\end{array}\right]} \\
{\left[y_{k}\right]=\left[\begin{array}{llll}
0 & 1 & 0 & 0
\end{array}\right]\left[\begin{array}{l}
x_{1, k} \\
x_{2, k} \\
a_{1, k} \\
b_{1, k}
\end{array}\right]+v_{k}}
\end{gathered}
$$

A covariância do ruído de modelo para esta nova modelagem é uma matriz bloco diagonal $\bar{Q}$, englobando nos blocos diagonais as matrizes $\overline{Q_{X}}$ e $\overline{Q_{P}}$, da seguinte forma:

$$
\begin{gathered}
\bar{Q}=\left[\begin{array}{cccc}
Q_{X} & 0 & 0 & 0 \\
0 & Q_{X} & 0 & 0 \\
0 & 0 & Q_{P a} & 0 \\
0 & 0 & 0 & Q_{P b}
\end{array}\right] \\
\overline{Q_{X}}=\left[\begin{array}{cc}
Q_{X} & 0 \\
0 & Q_{X}
\end{array}\right] \\
\overline{Q_{P}}=\left[\begin{array}{cc}
Q_{P a} & 0 \\
0 & Q_{P b}
\end{array}\right]
\end{gathered}
$$

sendo que $Q_{P a}$ e $Q_{P b}$ são os elementos da matriz de covariâncias $\overline{Q_{P}}$ associados aos parâmetros $a_{k}$ e $b_{k}$, respectivamente.

Neste modelo apresentado, supõe-se que o sistema elétrico esteja operando sem grandes perturbações em um determinado ponto de operação. Deste modo, o sistema é autônomo e excitado apenas pelos ruídos de modelagem do processo $w_{k}$ e $w_{p}$, em analogia às variações de carga no sistema [44,65]. As variáveis $w_{k}, w_{p}$ e $v_{k}$ são representadas no algoritmo FKE pelas matrizes de covariância $\bar{Q}$ e $\bar{R}$. 
Observa-se que o sistema obtido a partir da modelagem incluindo os parâmetros é não linear. O algoritmo do FKE, portanto, requer a matriz Jacobiana da matriz de transição de estados a cada iteração do algoritmo, definida como:

$$
A_{k}=\left[\begin{array}{cccc}
1 & -h a_{1, k} & -h x_{2, k} & 0 \\
h & 1-h b_{1, k} & 0 & -h x_{2, k} \\
0 & 0 & 1 & 0 \\
0 & 0 & 0 & 1
\end{array}\right]
$$

A matriz $C_{k}$ não depende de parâmetros variáveis, portanto, sua Jacobiana é a própria matriz de saída de (3.80).

$$
C_{k}=\left[\begin{array}{llll}
0 & 1 & 0 & 0
\end{array}\right]
$$

O intuito da aplicação ao se utilizar o FKE é identificar modos de oscilação dominantes observáveis nos sinais amostrados. No entanto, sabe-se que um sistema elétrico possui um número elevado de modos de oscilação, mas que apenas alguns deles são dominantes.

A modelagem na forma (3.74)-(3.75) considera que o sistema possui apenas um modo de oscilação, $\lambda=\sigma \pm j \omega$. No entanto, mais de um modo pode ser estimado pelo algoritmo de FKE estendendo-se a modelagem para quantos modos forem necessários, empilhando o sistema de espaço de estados (3.64)-(3.65) da seguinte forma:

$$
\begin{gathered}
{\left[\begin{array}{c}
\dot{x}_{1} \\
\dot{x}_{2} \\
\vdots \\
\dot{x}_{2 n-1} \\
\dot{x}_{2 n}
\end{array}\right]=\left[\begin{array}{ccccc}
0 & -a_{1} & \cdots & 0 & 0 \\
1 & -b_{1} & \cdots & 0 & 0 \\
\vdots & \vdots & \ddots & \vdots & \vdots \\
0 & 0 & \cdots & 0 & -a_{n} \\
0 & 0 & \cdots & 1 & -b_{n}
\end{array}\right]\left[\begin{array}{c}
x_{1} \\
x_{2} \\
\vdots \\
x_{2 n-1} \\
x_{2 n}
\end{array}\right]+w} \\
{[y]=\left[\begin{array}{lllll}
0 & 1 & \cdots & 0 & 1
\end{array}\right]\left[\begin{array}{c}
x_{1} \\
x_{2} \\
\vdots \\
x_{2 n-1} \\
x_{2 n}
\end{array}\right]+v}
\end{gathered}
$$

incluindo ainda as equações diferenciais dos parâmetros modelados como estados a serem estimados. Similarmente ao que foi feito para o caso com apenas um modo 
de oscilação na forma de espaço de estados em tempo discreto, obtém-se um sistema aumentado tal como a dedução efetuada para as equações (3.74)-(3.75).

A forma de cálculo dos parâmetros dos modos de oscilação no caso da modelagem com múltiplos modos é similar ao caso com apenas um modo, bastando calcular os autovalores da matriz de transição de estados dada por (3.81). Considerando que esta é uma matriz em blocos diagonais, os autovalores são facilmente calculados para cada bloco individualmente, da seguinte maneira:

$$
\lambda_{k}=-\frac{b_{k}}{2} \pm j \sqrt{a_{k}-\left(\frac{b_{k}}{2}\right)^{2}} \quad \text { para } \quad k=1,2, \ldots, n
$$

sendo:

$$
\begin{gathered}
\sigma_{k}=-\frac{b_{k}}{2} \\
\omega_{k}=\sqrt{a_{k}-\left(\frac{b_{k}}{2}\right)^{2}}
\end{gathered}
$$

\subsection{Ajustes do Filtro de Kalman}

Conforme foi apresentado nas seções 3.4, 3.4.1 e 3.4.2, o algoritmo do FK é um estimador ótimo de estados desde que os parâmetros do modelo sejam conhecidos, tais como as matrizes de transição de estados e de medições, bem como as covariâncias $Q$ e $R$ dos ruídos de modelo e de medição, respectivamente, e desde que os ruídos de modelo e de medição sejam caracterizados como ruídos brancos com média zero e distribuição normal.

No entanto, conforme modelagem do sistema proposta na seção 3.5, as matrizes de transição de estados e de medições são modelos cujos parâmetros se deseja determinar. O intuito neste trabalho é que os modos de oscilação estimados para os modelos sejam equivalentes aos modos dominantes reais presentes em um SEP. Os parâmetros que compõem as matrizes de transição a serem determinados não estão associados a nenhum parâmetro de um sistema real. Deste modo, do ponto de vista prático, é forçoso assumir que se tem um modelo desconhecido mas que os parâmetros deste modelo desconhecido têm covariâncias conhecidas [78].

Nesse sentido, foram feitos testes com o intuito de ajustar os parâmetros do FKE necessários à aplicação do filtro de forma que houvesse a rápida convergência dos valores 
a serem estimados e com pouca variabilidade. Os parâmetros do FKE a serem ajustados são os seguintes:

- covariâncias do ruído de modelo $Q\left(Q_{X}, Q_{P a}\right.$ e $\left.Q_{P b}\right)$;

- covariâncias do ruído de medição $R$;

- valor inicial do vetor de estados $\hat{x}_{0}$;

- valor inicial da matriz de covariâncias do erro de estimação de estados $P_{0}$;

- número de modos de oscilação a serem estimados $n$.

\subsubsection{Ajuste das Covariâncias $Q$ e $R$}

Por meio de diversos teste do algoritmo FKE, utilizando a modelagem proposta na seção 3.5, observou-se que o ajuste individual das covariâncias $Q$ e $R$ não era necessário, mas sim o ajuste relativo dessas covariâncias [74].

Verificou-se que, independente dos valores de $Q$ e $R$, caso a relação entre os dois valores seja mantida, os resultados são os mesmos. As Figuras 3.24, 3.25 e 3.26 ilustram a aplicação do algoritmo de FKE utilizando valores de $Q=R=0,01, Q=R=1$ e $Q=R=100$, respectivamente.

Conforme descrito na seção 3.5, a covariância do ruído de modelo para a modelagem proposta é uma matriz bloco diagonal $\bar{Q}$, englobando nos blocos diagonais as matrizes $\overline{Q_{X}}$ e $\overline{Q_{P}}$. Dessa forma, optou-se neste trabalho por fixar o valor da covariância de medição em $R=1$ e variar os valores de $Q_{X}$ e $Q_{P}$. Os valores da matriz $\overline{Q_{P}}$ foram ajustados de forma a refletir a covariância desejada dos parâmetros $a_{k}$ e $b_{k}$ descritos na Seção 3.5. Conforme diversos testes realizados de maneira empírica, os elementos da diagonal de $\overline{Q_{P}}$ associados aos parâmetros $b_{k}$ devem ser menores do que aqueles associados aos parâmetros $a_{k}$. Os parâmetros $b_{k}$ dizem respeito ao amortecimento do modo de oscilação a ser identificado, conforme equação (3.84), e são mais sensíveis a variações.

O ajuste global dos valores de $\bar{Q}$ relativamente aos de $\bar{R}$ irão impactar na capacidade de adaptação e na variação dos parâmetros dos modos de oscilação identificados pelo algoritmo. 

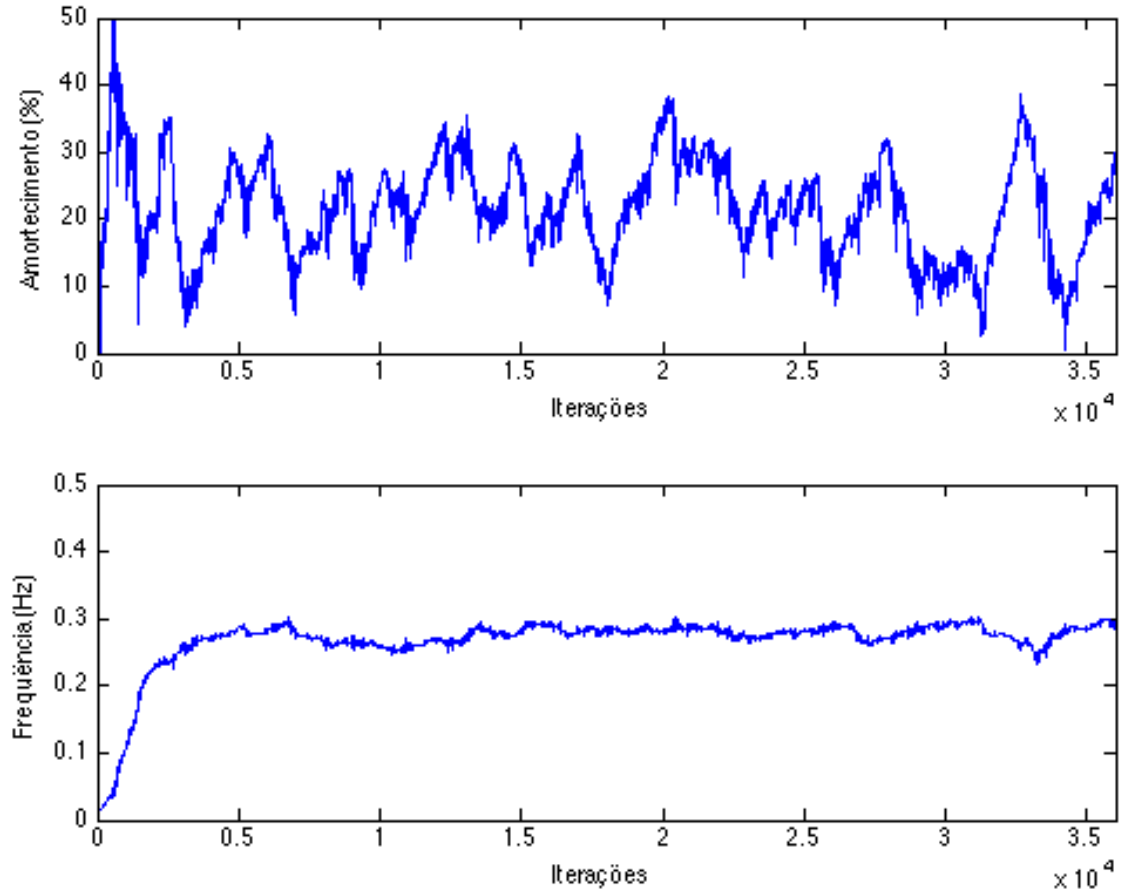

Figura 3.24: Aplicação do FKE para $Q=R=0,01$
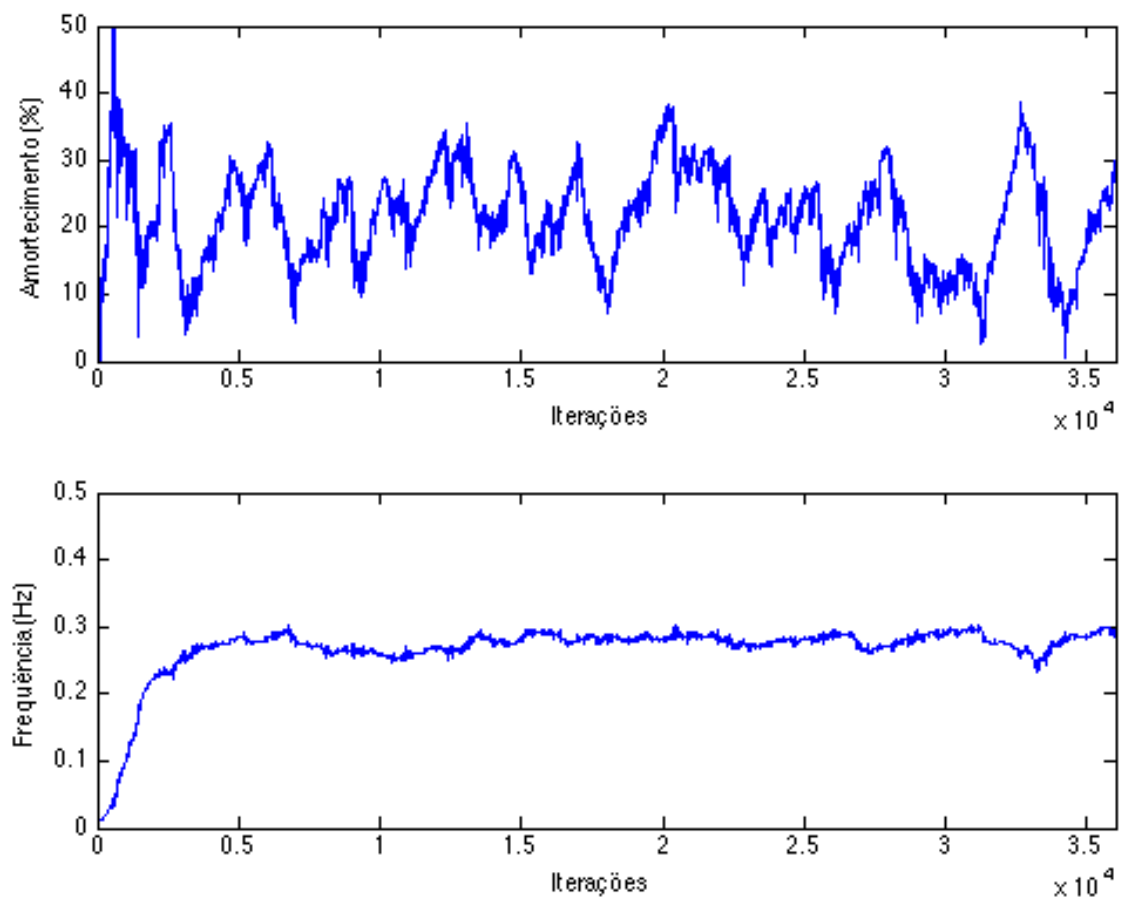

Figura 3.25: Aplicação do FKE para $Q=R=1$

3.6.2 Ajuste do valor inicial do vetor de estados, $\hat{x}_{0}$, e da matriz de covariâncias do erro de estimação de estados, $P_{0}$

Também por meio de inúmeros testes realizados com o algoritmo FKE, utilizando a modelagem proposta na seção 3.5 , constatou-se que os valores de $\hat{x}_{0}$ e de $P_{0}$ são deter- 

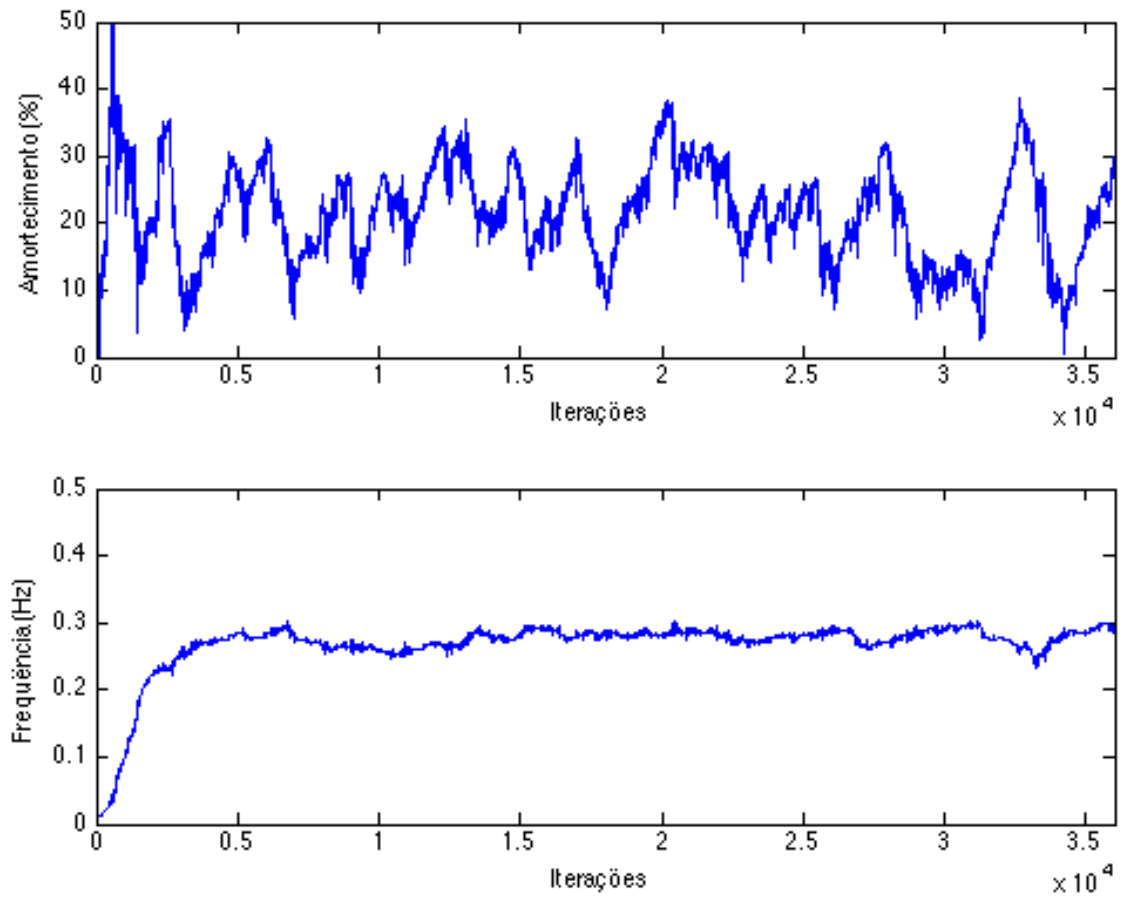

Figura 3.26: Aplicação do FKE para $Q=R=100$

minantes para a convergência do FKE. No entanto, desde que ocorra a convergência, os valores de $\hat{x}_{0}$ e de $P_{0}$ têm pouca influência em relação aos valores dos estados $\hat{x}$ após algumas iterações.

A Figura 3.27 ilustra a convergência dos resultados. Nela são plotadas as curvas de amortecimento e frequência para realizações do algoritmo de FKE para vários valores de $\hat{x}_{0}$ e de $P_{0}: \hat{x}_{0}=0$ e $P_{0}=0$ em azul; $\hat{x}_{0}=100$ e $P_{0}=0$ em vermelho; $\hat{x}_{0}=100$ e $P_{0}=100 I_{4 p}$ em verde; $\hat{x}_{0}$ randômico e $P_{0}=0$ em ciano; $\hat{x}_{0}$ randômico e $P_{0}=100 I_{4 p}$ em preto, em que $I_{j}$ é a matriz identidade de ordem $j$ e $p$ é o número de modos a serem estimados pelo modelo.

Verifica-se que, para todas as combinações de valores de $\hat{x}_{0}$ e $P_{0}$, os resultados do algoritmo de FKE para a modelagem proposta neste trabalho convergem para os mesmos valores. A diferença verificada é que esta convergência poderá ser retardada, por exemplo, nos casos da curvas identificadas em vermelho e em verde.

Assim, neste trabalho optou-se por adotar os seguintes valores iniciais: $\hat{x}_{0}=0 \mathrm{e}$ $P_{0}=I_{4 p}$. 

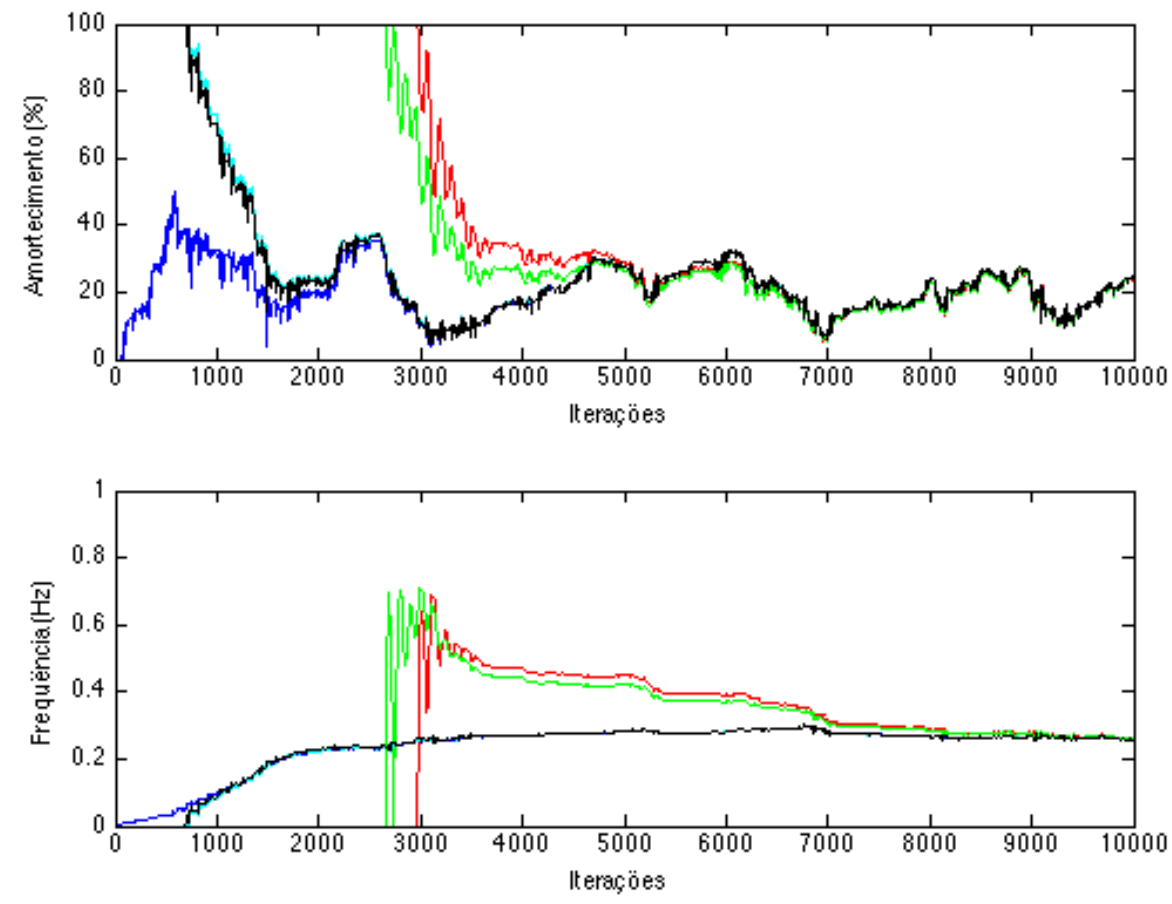

Figura 3.27: Aplicação do FKE para vários valores de $\hat{x}_{0}$ e de $P_{0}$

\subsubsection{Ajuste do número de modos de oscilação a serem estimados}

A definição do número de modos de oscilação a serem estimados é sempre um problema quando trata-se de identificação dos modos a partir de dados de ambiente coletados por meio de PMUs [2].

Os SEPs possuem elevado número de modos de oscilação, mas apenas alguns são dominantes e observáveis por meio de medições de PMUs. Tipicamente, um sinal medido não possui mais do que seis modos observáveis [2]. Dependendo do sinal analisado, os modos podem ser melhor observados em um sinal do que em outro [34].

Os métodos de identificação paramétricos irão, invariavelmente, tentar identificar uma quantidade de modos de oscilação inferior à quantidade realmente existente no sistema. O objetivo, no entanto, deve ser tentar identificar os modos em quantidade tal que melhor represente a dinâmica do sinal analisado.

Se o modelo proposto tiver menos modos do que o ideal para representar o sinal analisado, a identificação ficará incompleta e os modos identificados não representarão os modos presentes no sinal, havendo perda de informação. Por outro lado, se o modelo tiver mais modos do que o ideal, irão ser identificados modos espúrios e inexistentes devido aos ruídos presentes no sinal [2]. 
Para determinar o número de modos de oscilação a serem identificados em dados de ambiente, alguns autores utilizam as técnicas chamadas Akaike Information Criterion (AIC) e Final Prediction Error (FPE), outros calculam a quantidade de modos a partir dos valores singulares da matriz de Hankel do sinal, e outros procedem por tentativa e erro. Geralmente, os modelos utilizados identificam de 20 a 25 modos de oscilação, sendo que alguns trabalhos utilizam modelos com 45 ou até 65 modos e aplicam alguma técnica para separar os modos dominantes daqueles que são fracos ou espúrios $[2,50,51,63]$.

Neste trabalho optou-se por definir a quantidade de modos a serem identificados visualmente com base no espectro de frequências de cada sinal, calculado por meio da Transformada Rápida de Fourier (Fast Fourier Transform - FFT), um método não paramétrico de análise de sinais. Os modos dominantes em geral são modos lentos, com menor amortecimento, e são bem caracterizados por picos no espectro de frequências dos sinais $[2]$.

Procedendo desta forma, utiliza-se um método não paramétrico para validar os resultados encontrados quanto ao número de modos de oscilação identificados utilizando o FKE, um método paramétrico. Muitos autores recomendam esse tipo de comparação como método de verificação de resultados [2].

Como exemplo, a Figura 3.28 apresenta o espectro de frequências de um sinal simulado de dados de ambiente com um único pico em 0,288 Hz. O modo de oscilação dominante deste sinal possui frequência de $0,29 \mathrm{~Hz}$ e amortecimento de $9,23 \%$, conforme dados da simulação.

Neste caso, para definir a quantidade de modos de oscilação que deverão ser identificados, as frequências dos modos identificados deverão ser comparadas com a do pico presente no espectro de frequências do sinal.

A Figura 3.29 apresenta um exemplo hipotético da forma de definição da quantidade de modos de oscilação que devem ser identificados para o caso do sinal da Figura 3.28.

A Figura 3.29 apresenta três espectros de frequências do sinal juntamente com marcadores em vermelho. Em a) observa-se em vermelho a frequência do modo identificado utilizando o modelo com apenas um modo, em b) o modelo possui dois modos destacados em vermelho e em c) o modelo possui três modos de oscilação. 


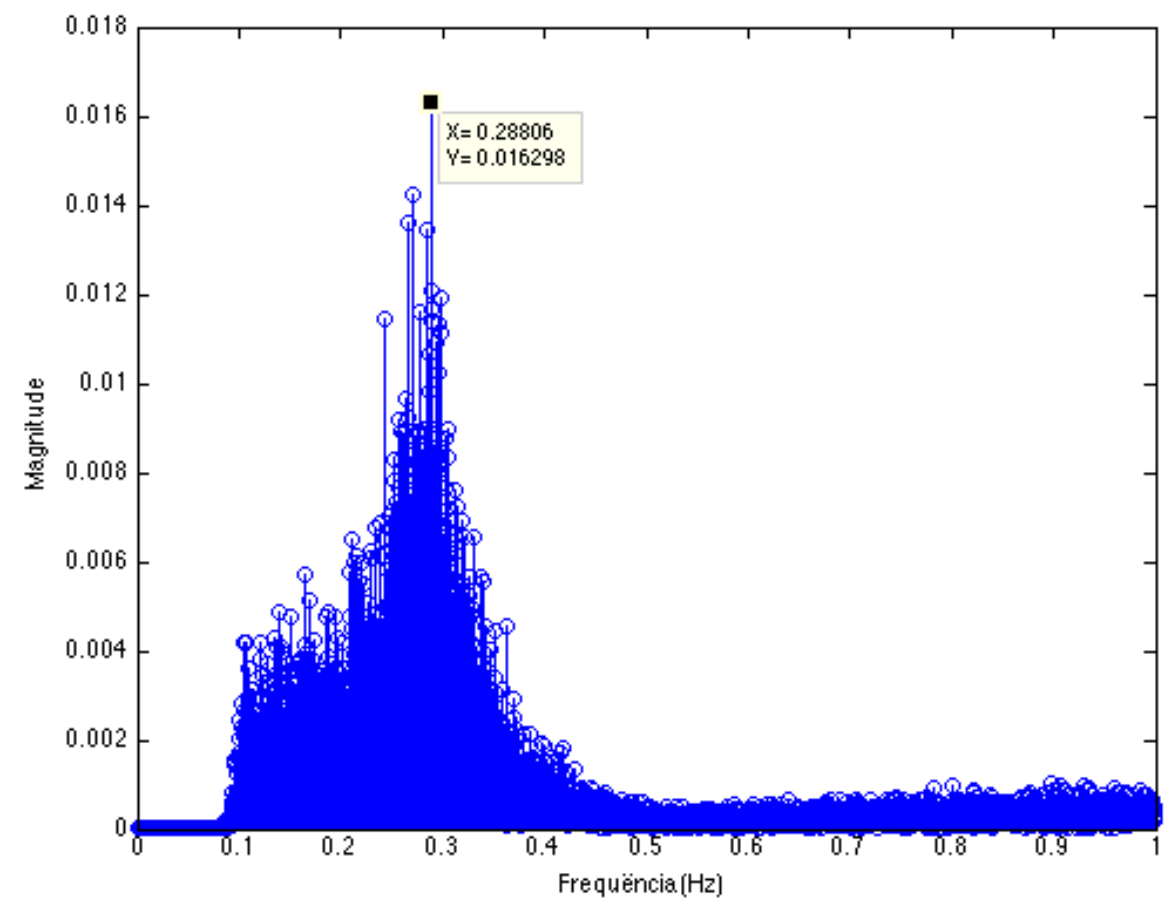

Figura 3.28: Espectro de frequências de um sinal de dados de ambiente

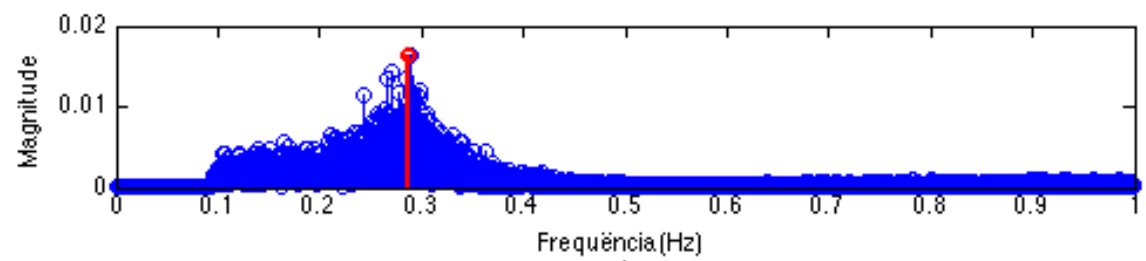

a)

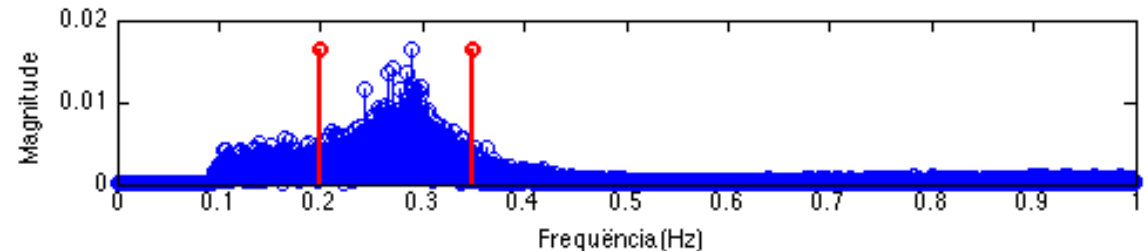

b]

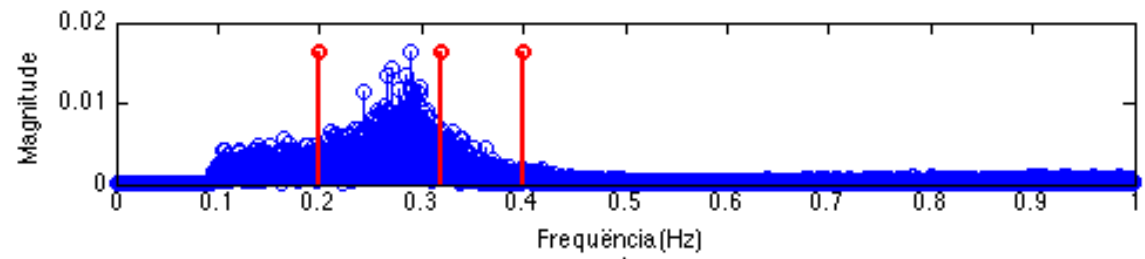

c)

Figura 3.29: Espectro de frequências de um sinal de dados de ambiente e as frequências de modos hipotéticos identificados: a) um modo de oscilação identificado; b) dois modos de oscilação identificados; c) três modos de oscilação identificados

Da análise da figura conclui-se que o modelo a), com apenas um modo de oscilação, foi o que identificou o modo com frequência coerente com o pico do espectro de frequências. Neste exemplo seria escolhido o modelo a), com apenas um modo de oscilação, para 
identificar o modo de oscilação dominante do sinal.

\subsection{Conclusão}

Neste capítulo foram apresentadas as PMUs, com destaque para o Projeto Medfasee. Foram descritas as etapas do pré-processamento dos sinais coletados nas PMUs, tais como interpolação, ajustes angular e filtragem. Foram apresentadas também as formulações matemáticas do FK e do FKE e feitos os ajustes dos parâmetros iniciais do FKE para aplicação do algoritmo proposto neste trabalho. E, por fim, foi detalhada a modelagem em sistema de espaço de estados proposta nesta dissertação para identificação de modos de oscilação dominantes de SEP utilizando o FKE.

Os ajustes dos parâmetros iniciais $Q, R, \hat{x}_{0}$ e $P_{0}$ foram realizados de maneira a aumentar a capacidade de adaptação e diminuir a variabilidade dos resultados do algoritmo de FKE. Os valores de $R, \hat{x}_{0}$ e $P_{0}$ foram escolhidos após testes como sendo iguais a 1,0 e $I_{4 p}$, respectivamente. Já os valores de $Q_{X}, Q_{P a}$ e $Q_{P b}$ foram determinados de acordo com o sinal estudado, sendo que $Q_{P b}$ deve ser muito menor do que $Q_{P a}$.

No próximo capítulo são apresentados os resultados da aplicação da metodologia proposta em sinais simulados por meio do software PST e em dados coletados de PMUs do SIN. 


\section{Testes e Resultados}

\subsection{Introdução}

Os capítulos anteriores trataram do pré-processamento dos dados de PMUs, do método de FKE e da modelagem em espaço de estados para identificação de modos dominantes em SEPs. Agora são apresentados os resultados da aplicação desta metodologia em sinais simulados por meio do software PST e em dados coletados de PMUs do SIN. O objetivo é avaliar a capacidade da metodologia em identificar modos de oscilação eletromecânica dominantes em SEPs a partir de medições fasoriais sincronizadas.

Com esta finalidade, os conteúdos deste capítulo são apresentados da seguinte forma: na seção 4.2 é apresentado o sistema teste utilizado nas simulações e são descritos os resultados da aplicação da metodologia proposta em sinais simulados, e na seção 4.3 são apresentados os resultado da aplicação do método proposto em sinais de diferença angular de tensão coletados por meio de PMUs instaladas no SIN. Na seção 4.3.1 são tratados sinais de diferença angular cuja referência é o ângulo de tensão da PMU da UFC, na seção 4.3.2 sinais cuja referência é a PMU da UFT, e na seção 4.3 .3 sinais cuja referência é a PMU da UFMA.

\subsection{Simulações}

Para melhor ilustrar a eficácia da metodologia proposta nesta dissertação, foram efetuados testes seguindo duas abordagens: uma em que se dispõe do modelo do sistema e, portanto, os sinais de PMU são simulados, descrita nesta seção; uma outra, em que se dispõe apenas dos sinais de PMUs coletados no SIN, caso prático, em que não se conhece o modelo do sistema, descrita na próxima seção.

\subsubsection{Sistema teste para simulações}

Testes foram realizados em um sistema composto por duas áreas interconectadas através de duas linhas de transmissão longas. Em cada área existem dois geradores, nas barras 1 e 2 na área 1, e nas barras 11 e 12 na área 2. As cargas estão localizadas na barra 4 da área 1 e na barra 14 da área 2. A barra 1 atua como barra de referência. A Figura 4.1 apresenta o diagrama unifilar deste sistema. 


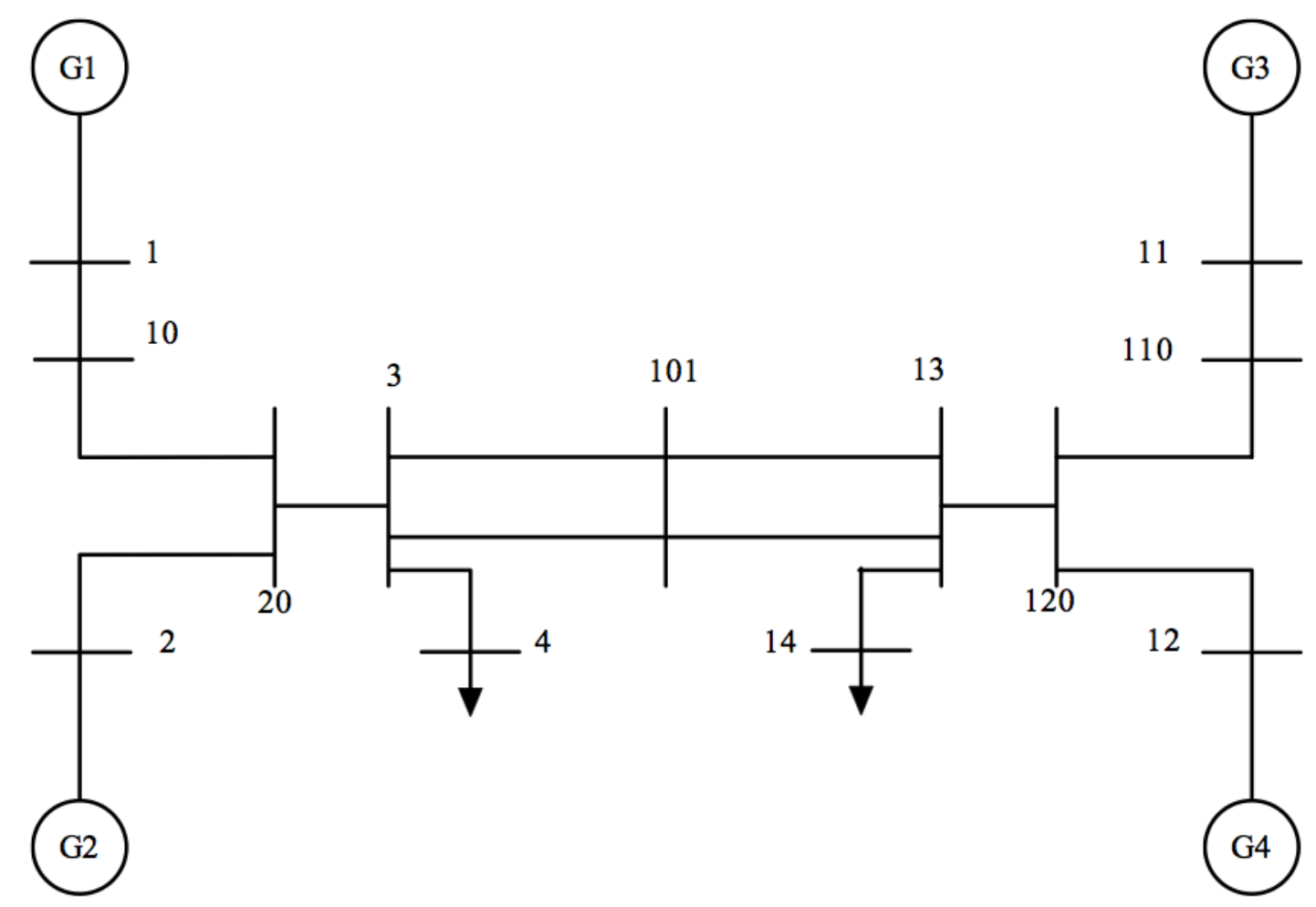

Figura 4.1: Diagrama unifilar do sistema teste no PST

Os testes foram realizados com o auxílio do software livre Power System Toolbox (PST) [79], desenvolvido por Joe Chow, Kwok W. Cheung e Graham Rogers, e disponível para download em [80]. O PST funciona em conjunto com o Matlab e é utilizado para realizar estudos de fluxo de potência, bem como de estabilidade de SEP.

Todos os geradores do sistema teste foram modelados utilizando um modelo subtransitório. Os geradores são dotados de modelos dinâmicos de regulador de tensão e PSS. Todas as cargas ativas são modeladas como 50\% de corrente constante e $50 \%$ de impedância constante.

Em todas as barras com carga, acrescentou-se à potência ativa parcela variando aleatoriamente com distribuição Gaussiana na proporção de 1\%. A finalidade foi simular o efeito do ruído ambiente no SEP em estudo. Foram simuladas medições de fasores de tensão nas barras 3 e 13, a uma taxa de 60 amostras por segundo. Esta é a mesma taxa de amostragem utilizada nas PMUs instaladas no âmbito do Projeto Medfasee, cuja análise é apreciada mais à frente.

Foram simulados cinco casos diferentes, sendo quatro casos em que os modos de oscilação do sistema permanecem inalterados durante toda a simulação e um caso em que o modo de oscilação varia ao longo da simulação. 
Em todos os casos foram efetuadas simulações de Monte Carlo, caracterizadas por 100 realizações com janelas de 60 minutos de duração cada. Todas as realizações foram produzidas no software PST. A cada realização, a frequência e o amortecimento dos modos de oscilação, quando modificados, foram alterados a partir de ajustes nos valores do momento de inércia dos geradores e do ganho dos PSSs.

O FKE foi aplicado, em todos os casos, com as seguintes matrizes de covariâncias: $P_{0}=I_{4 p}, \overline{Q_{X}}=100 I_{2 p}$ (no quarto e quinto casos foi utilizado $\overline{Q_{X}}=50 I_{2 p}$ na modelagem com dois modos), $Q_{P a}=20, Q_{P b}=0,5$ e $\bar{R}=I_{1}$, em que $I_{j}$ é a matriz identidade de ordem $j, p$ é o número de modos a serem estimados pelo modelo e $Q_{P a}$ e $Q_{P b}$ são os elementos da matriz de covariâncias $\overline{Q_{P}}$ associados aos parâmetros $a_{k}$ e $b_{k}$, respectivamente. Todos os estados $x_{0}$, incluindo aqueles representativos dos parâmetros utilizados para calcular os modos de oscilação, foram inicializados com valores iguais a zero. Apesar da inicialização ser feita desta forma, nenhum problema foi identificado com relação à convergência final de parâmetros.

Os casos são analisados nas subseções seguintes.

\subsubsection{Primeiro caso - um modo dominante fixo}

O primeiro caso simulado utilizando o sistema teste da Figura 4.1 possui o modo de oscilação fixo descrito na Tabela 4.1. Trata-se do modo de oscilação entre as duas áreas do sistema teste.

Tabela 4.1: Modo de oscilação constante do primeiro caso.

\begin{tabular}{c|c}
\hline Frequência $(\mathrm{Hz})$ & Amortecimento $(\%)$ \\
\hline 0,2908 & 9,228 \\
\hline
\end{tabular}

O algoritmo de FKE proposto no Capítulo 3 foi aplicado para 100 simulações realizadas por meio do PST. Após testes utilizando modelos com várias quantidades de modos, optou-se por utilizar neste primeiro caso simulado, assim como no segundo e terceiro casos descritos nas próximas seções, um modelo constituído por apenas um modo. No quarto e quinto casos, como será visto, o mais adequado foi a utilização de modelo com dois modos.

A Figura 4.2 apresenta a média dos valores encontrados para frequência e amortecimento do modo de oscilação ao longo do período de uma hora de simulação, considerando as 100 simulações realizadas, bem como o desvio padrão desses valores. As linhas 
azuis indicam os valores médios, e as linhas vermelhas representam o desvio padrão para mais e para menos desses valores. As linhas pretas retas, indicando valores constantes nos gráficos, servem de referência para definição dos valores das grandezas identificadas de frequência e amortecimento.
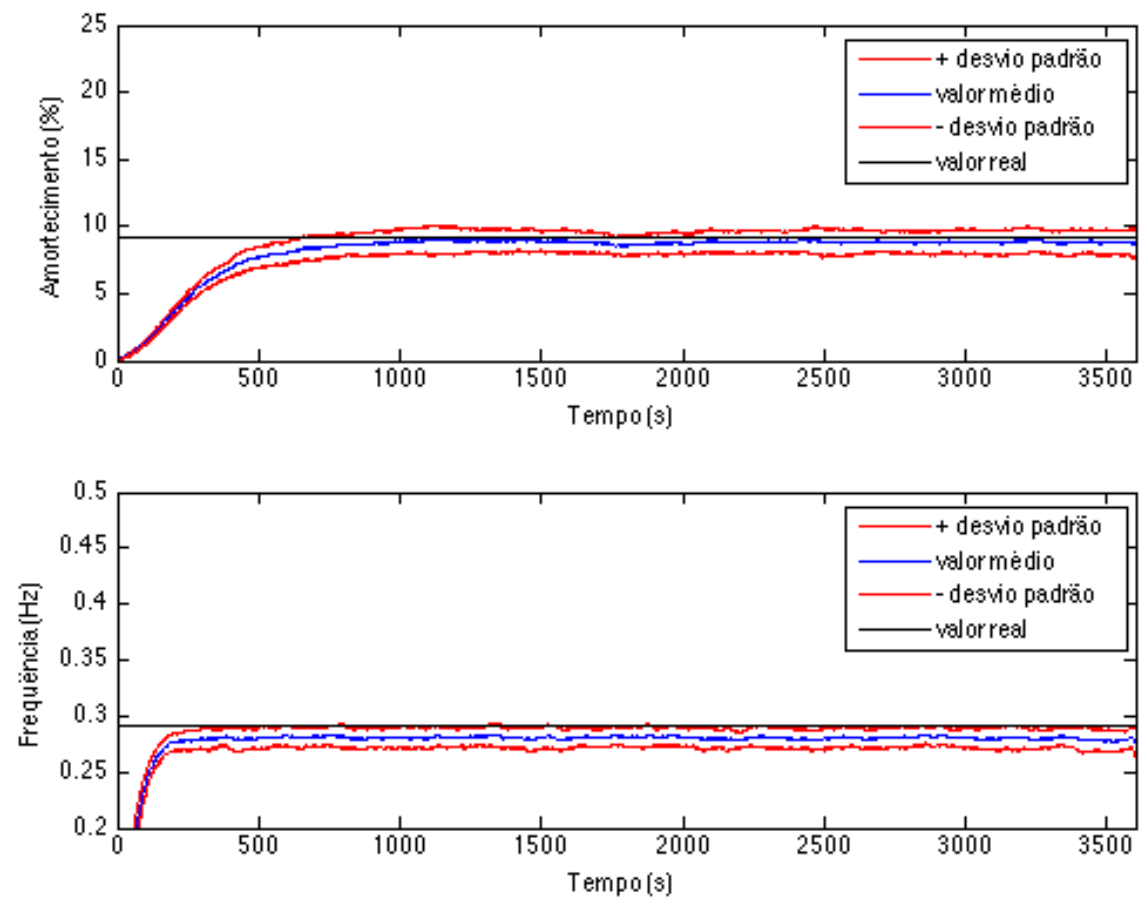

Figura 4.2: Frequência e amortecimento do modo de oscilação para o primeiro caso

Observa-se na Figura 4.2 que o algoritmo converge após aproximadamente 10 minutos de sinal. A identificação do amortecimento e da frequência são ligeiramente inferiores aos valores reais.

A Figura 4.3 apresenta o espectro de frequências deste sinal, considerando as 100 simulações realizadas, com a frequência do modo de oscilação identificado destacada em vermelho. Verifica-se que a frequência tem valor próximo àquela do pico do espectro de frequências do sinal, o que indica que a modelagem com apenas um modo de oscilação foi adequada para a identificação do modo de oscilação interárea deste sinal.

\subsubsection{Segundo caso - um modo dominante fixo}

O segundo caso simulado possui o modo de oscilação fixo descrito na Tabela 4.2. Tratase do modo de oscilação entre as duas áreas do sistema teste, mas com amortecimento inferior em relação ao amortecimento simulado no primeiro caso. Para tanto, os valores de ganho dos PSSs dos geradores síncronos do sistema teste foram alterados. 


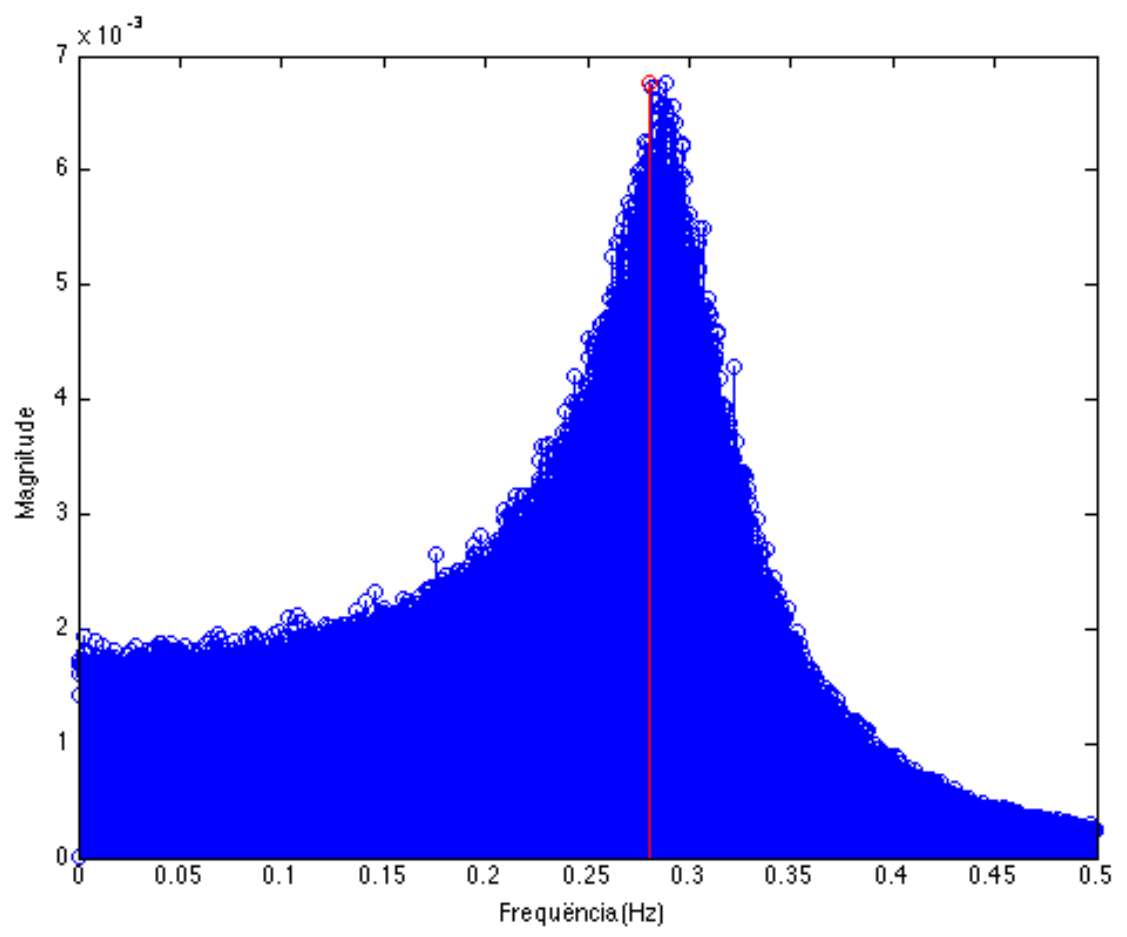

Figura 4.3: Espectro de frequências do sinal simulado no primeiro caso com destaque para a frequência do modo de oscilação identificado

Tabela 4.2: Modo de oscilação constante do segundo caso.

\begin{tabular}{c|c}
\hline Frequência $(\mathrm{Hz})$ & Amortecimento (\%) \\
\hline 0,2949 & 3,288 \\
\hline
\end{tabular}

Novamente, o algoritmo de FKE foi aplicado para 100 simulações e foi utilizado um modelo constituído de apenas um modo.

A Figura 4.4 apresenta a média dos valores encontrados para frequência e amortecimento do modo de oscilação ao longo do período de uma hora de simulação, considerando as 100 simulações realizadas, bem como o desvio padrão desses valores. O padrão de cores desta figura é o mesmo daquele adotado para a Figura 4.2 do primeiro caso simulado.

Assim como no primeiro caso, observa-se na Figura 4.4 que o algoritmo converge após aproximadamente 5 minutos, com valores de amortecimento e frequência identificados ligeiramente diferentes dos valores reais.

A Figura 4.5 apresenta o espectro de frequências deste sinal, considerando as 100 simulações realizadas, com a frequência do modo de oscilação identificado destacada em 

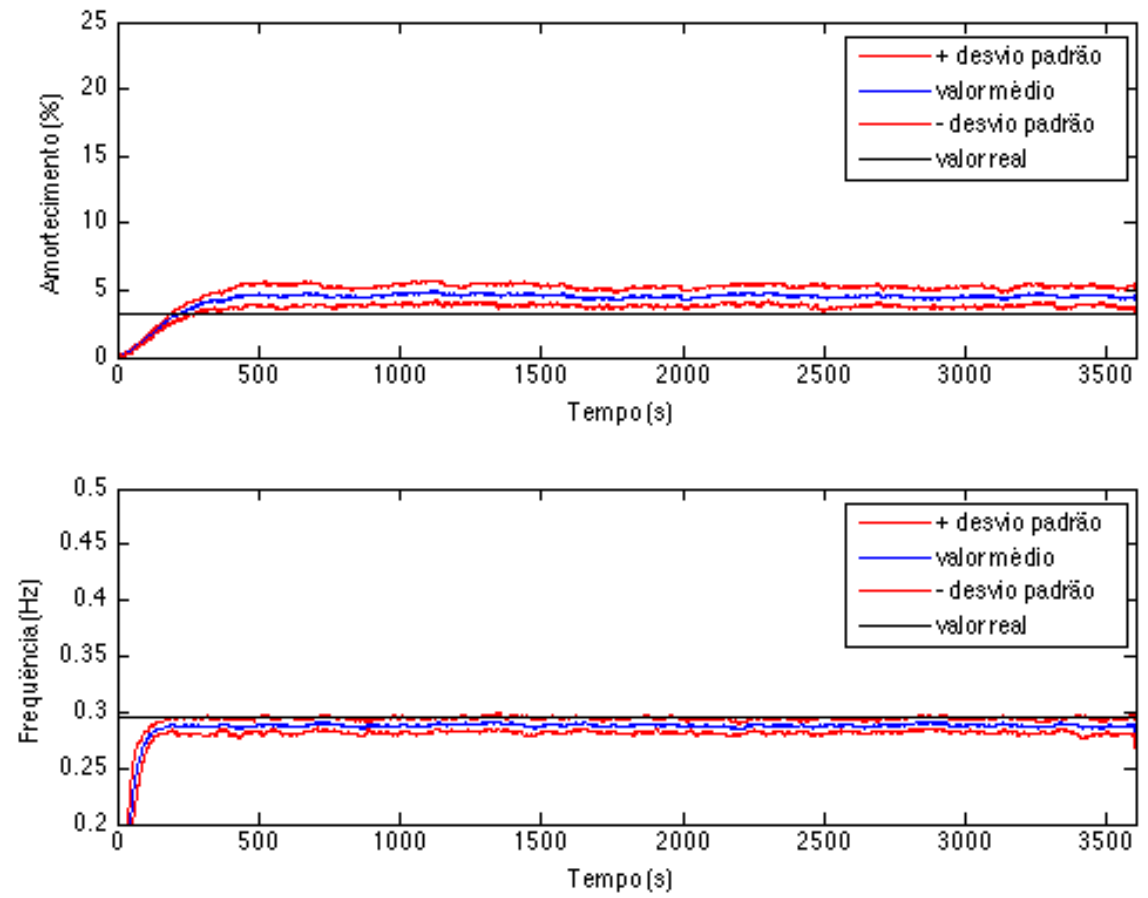

Figura 4.4: Frequência e amortecimento do modo de oscilação identificado no segundo caso

vermelho. Verifica-se que a frequência tem valor próximo àquela do pico do espectro de frequências do sinal, o que indica que a modelagem com apenas um modo de oscilação também foi adequada para a identificação do modo de oscilação interárea deste sinal.

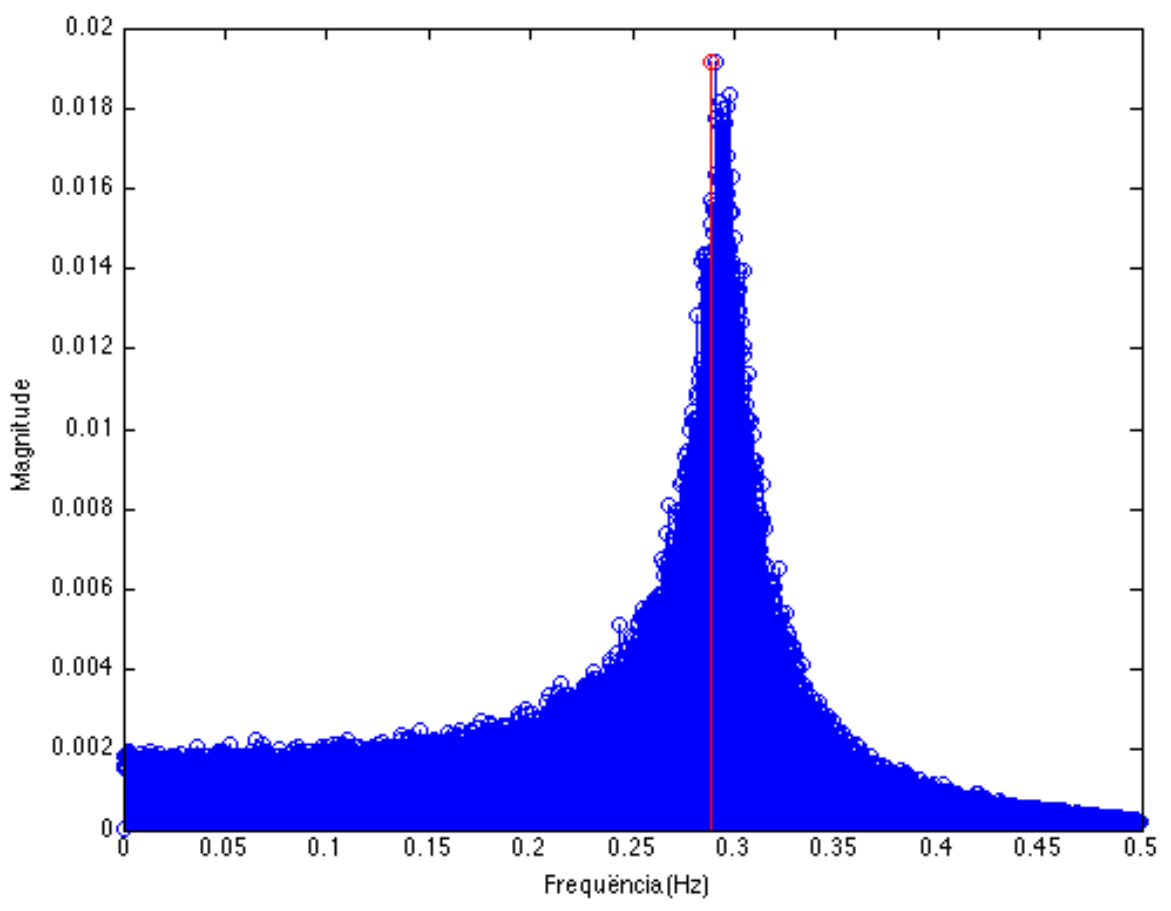

Figura 4.5: Espectro de frequências do sinal simulado no segundo caso com destaque para a frequência do modo de oscilação identificado 


\subsubsection{Terceiro caso - um modo dominante variável}

O terceiro caso simulado é uma composição dos dois primeiros e possui um modo de oscilação nos primeiros 30 minutos de simulação e outro, com amortecimento menor, nos 30 minutos seguintes. Esses dois modos de oscilação se alternam de 30 em 30 minutos durante duas horas e meia de simulação. Os parâmetros dos modos estão descritos na Tabela 4.3. Trata-se do modo de oscilação entre as duas áreas do sistema teste. Para que fosse possível alterar o amortecimento do modo, alterou-se os ganhos dos PSS dos geradores síncronos ao longo da simulação.

Tabela 4.3: Modo de oscilação variável do terceiro caso.

\begin{tabular}{c|c|c}
\hline Tempo $(\mathrm{hr})$ & Frequência $(\mathrm{Hz})$ & Amortecimento $(\%)$ \\
\hline $0-0,5$ & 0,2908 & 9,228 \\
$0,5-1$ & 0,2949 & 3,288 \\
$1-1,5$ & 0,2908 & 9,228 \\
$1,5-2$ & 0,2949 & 3,288 \\
$2-2,5$ & 0,2908 & 9,228 \\
\hline
\end{tabular}

A aplicação do algoritmo de FKE foi realizada da mesma forma que nos dois primeiros casos, com um modelo de apenas um modo de oscilação.

A Figura 4.6 apresenta a média dos valores encontrados para frequência e amortecimento do modo de oscilação ao longo do período de duas horas e meia de simulação, considerando as 100 simulações realizadas, bem como o desvio padrão desses valores. As linhas pretas retas indicando valores constantes a cada intervalo de 30 minutos nos gráficos servem de referência para definição dos valores das grandezas identificadas de frequência e amortecimento.

Observa-se na Figura 4.6 que o algoritmo converge em menos de 10 minutos no primeiro trecho de 30 minutos do sinal. Após a transição para o segundo trecho, em que o amortecimento do modo de oscilação é reduzido, a convergência para o novo valor de amortecimento também ocorre em poucos minutos. E assim ocorre em todas as transições entre os trechos.

Assim como nos dois primeiros casos simulados, os valores identificados para o amortecimento e a frequência são ligeiramente diferentes dos valores reais. 

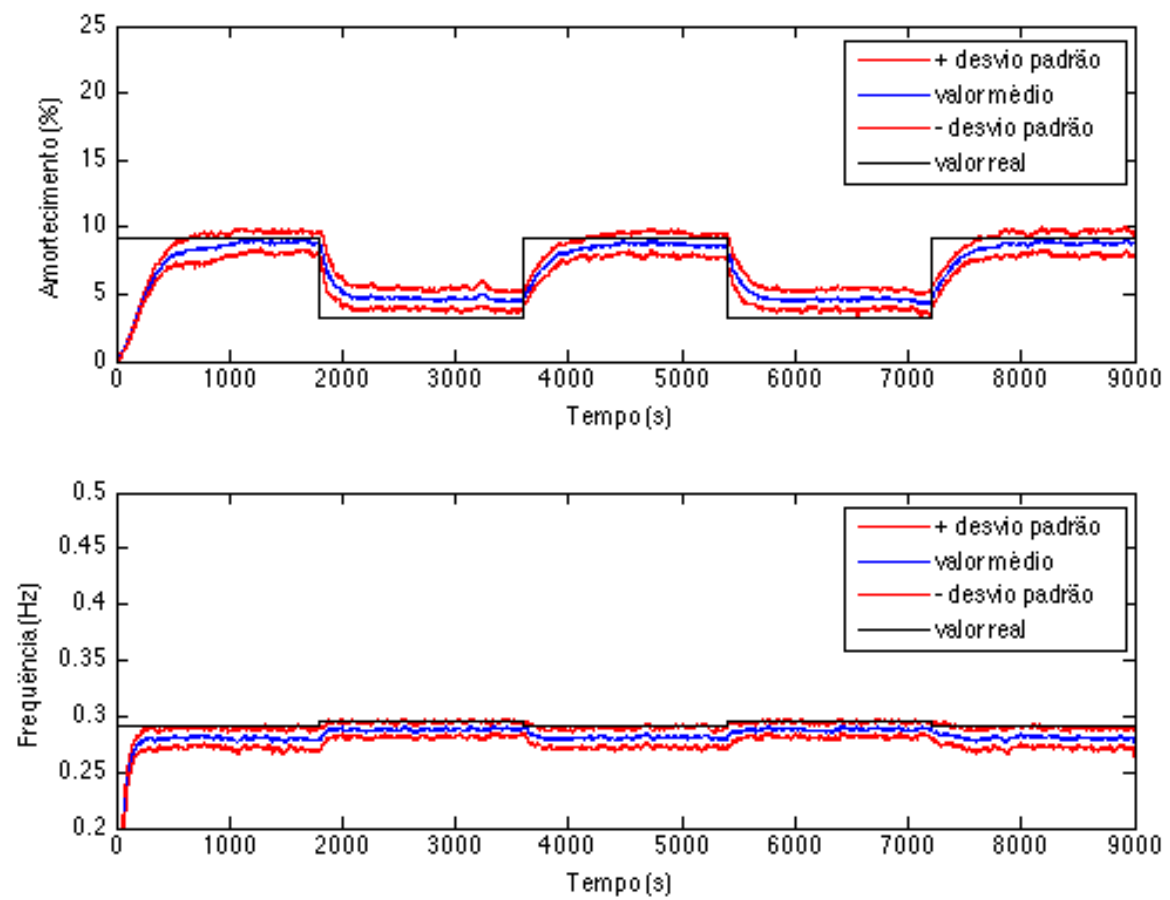

Figura 4.6: Frequência e amortecimento do modo de oscilação variável identificado no terceiro caso

\subsubsection{Quarto caso - um modo dominante fixo}

No quarto e no quinto casos simulados, a frequência do modo de oscilação dominante no sistema foi alterada por meio da modificação da constante de inércia dos geradores síncronos do sistema teste em relação aos casos anteriores. O modo de oscilação fixo para este quarto caso está descrito na Tabela 4.4. Trata-se do modo de oscilação entre as duas áreas do sistema teste.

Tabela 4.4: Modo de oscilação constante do quarto caso.

\begin{tabular}{c|c}
\hline Frequência $(\mathrm{Hz})$ & Amortecimento $(\%)$ \\
\hline 0,4339 & 11,87 \\
\hline
\end{tabular}

O algoritmo de FKE proposto no Capítulo 3, assim como nos casos anteriores, foi aplicado para 100 simulações realizadas por meio do PST. Inicialmente utilizou-se a modelagem com apenas um modo de oscilação.

A Figura 4.7 apresenta a média dos valores encontrados para frequência e amortecimento do modo de oscilação ao longo do período de uma hora de simulação, considerando as 100 simulações realizadas, bem como o desvio padrão desses valores. O padrão de cores desta figura é o mesmo daquele adotado para o primeiro caso simulado. 

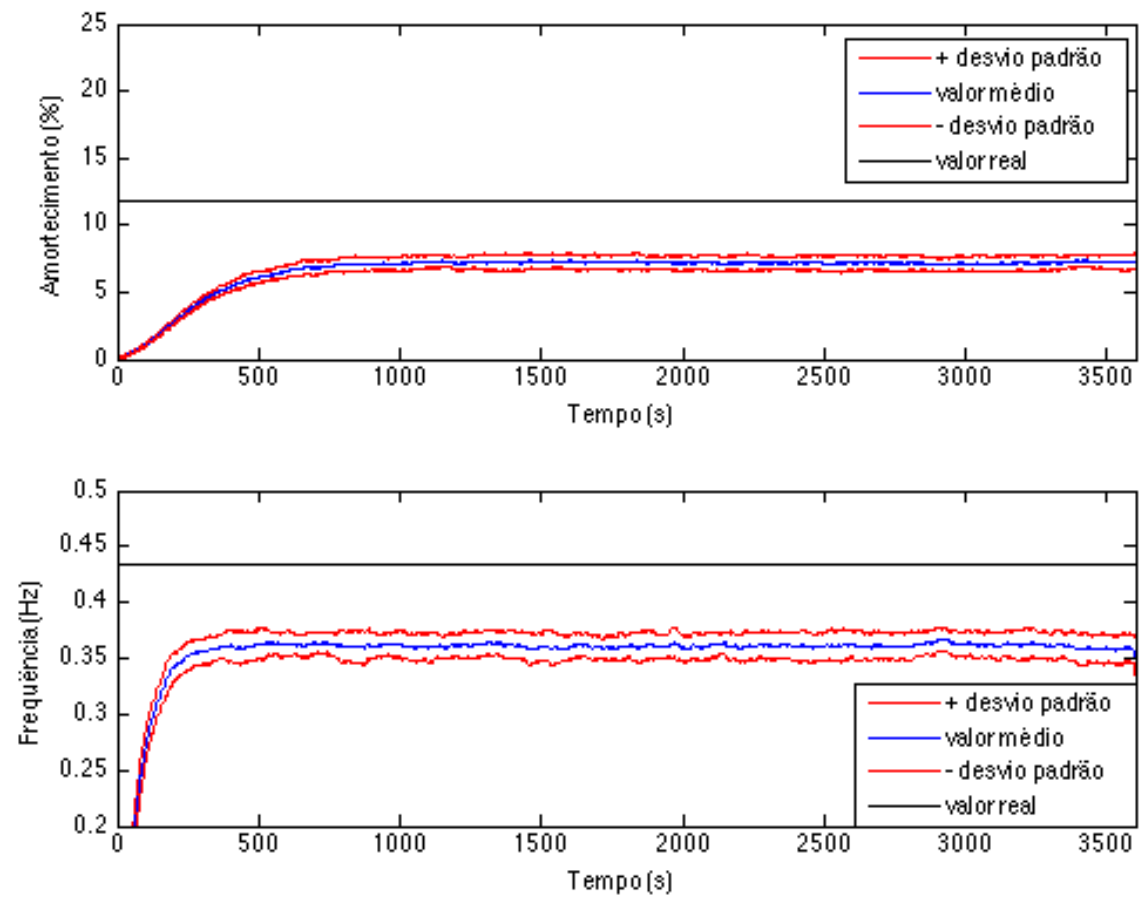

Figura 4.7: Frequência e amortecimento do modo de oscilação identificado no quarto caso utilizando modelagem com um modo de oscilação

Observa-se na Figura 4.7 que o algoritmo converge em aproximadamente 10 minutos, mas os valores encontrados para frequência e amortecimento do modo de oscilação não estão coerentes com os valores reais. Ao analisar o espectro de frequências deste sinal, considerando as 100 simulações realizadas, como pode ser visto na Figura 4.8, percebese que a frequência do modo identificado é bem diferente da frequência da componente do pico de magnitude no espectro de frequências. Conclui-se que a quantidade de modos de oscilação identificada pode ter sido insuficiente para representar o comportamento dinâmico do sinal.

Aplicou-se novamente o algoritmo de FKE, mas dessa vez utilizando a modelagem com dois modos de oscilação. A Figura 4.9 apresenta o espectro de frequências do sinal, considerando as 100 simulações realizadas, com destaque nas frequências dos dois modos de oscilação identificados.

Percebe-se pela Figura 4.9 que o modo de oscilação com maior frequência identificado está coerente com o pico de magnitude do espectro de frequências do sinal. A Figura 4.10 apresenta os valores de frequência e amortecimento identificados associados a este modo de oscilação de maior frequência.

Como pode ser visto na Figura 4.10, utilizando a modelagem com dois modos de os- 


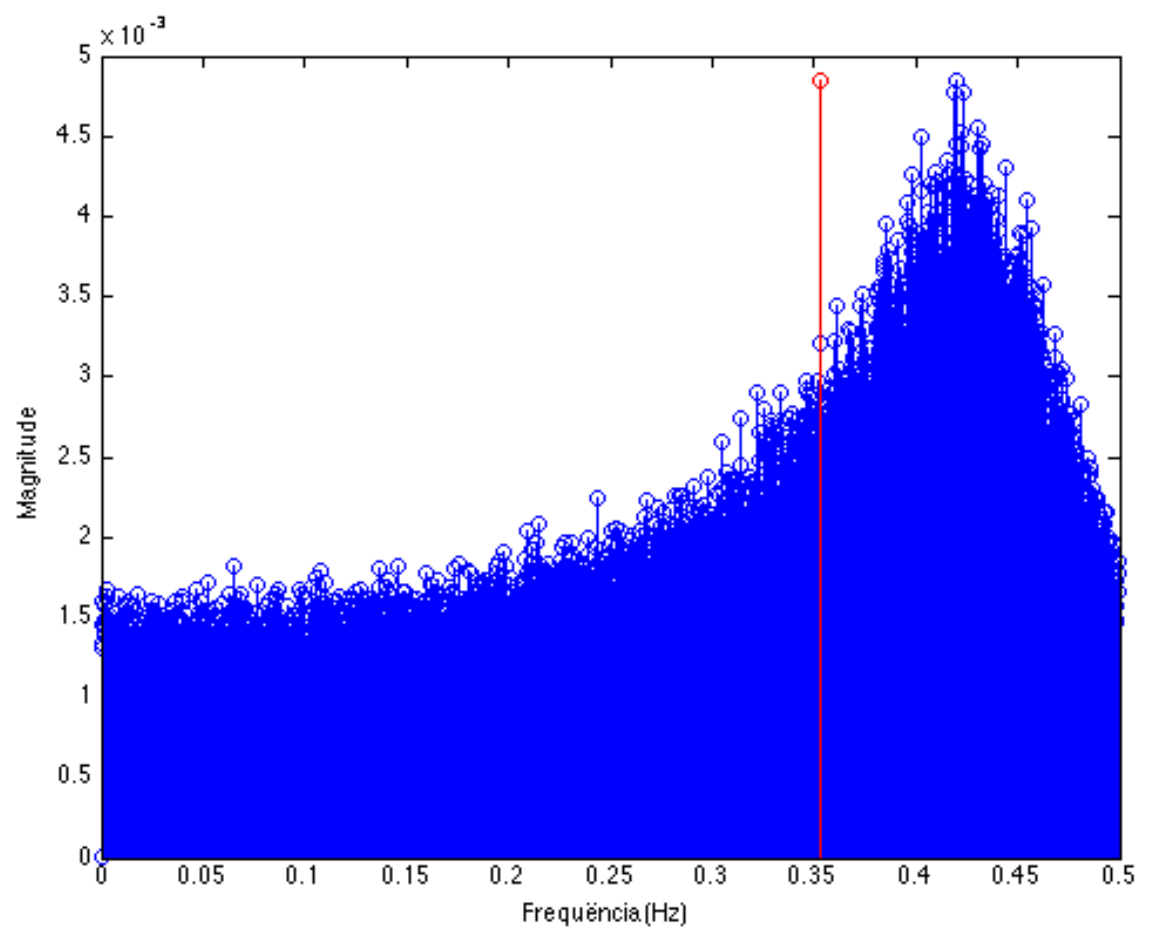

Figura 4.8: Espectro de frequências do sinal simulado no quarto caso utilizando um modelo com um modo com destaque para a frequência do modo de oscilação identificado

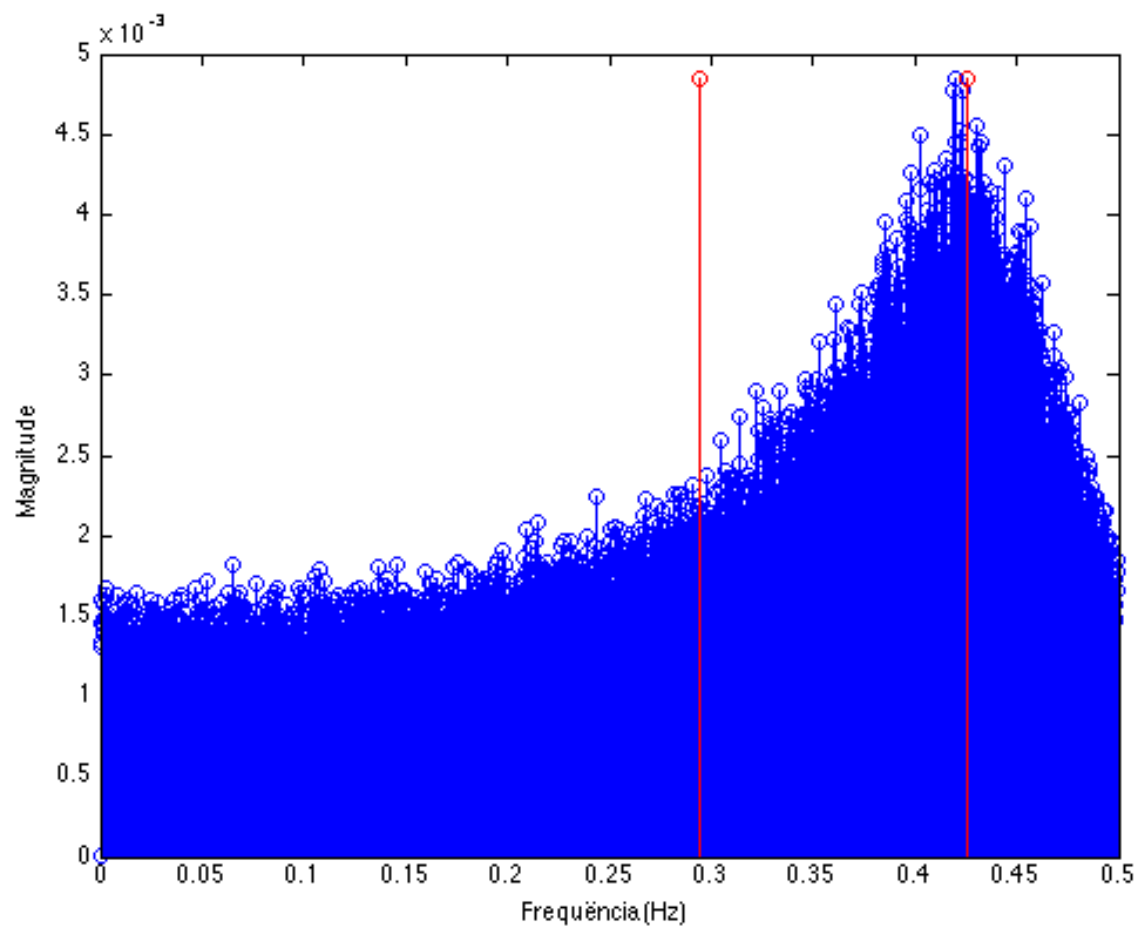

Figura 4.9: Espectro de frequências do sinal simulado no quarto caso utilizando um modelo com dois modos com destaque para as frequências dos dois modos de oscilação identificados

cilação, os valores de frequência e amortecimento do modo de oscilação identificado são coerentes com os valores reais. Os valores de amortecimento identificados foram ligeiramente inferiores aos valores reais, assim como os valores de frequência. Vários 

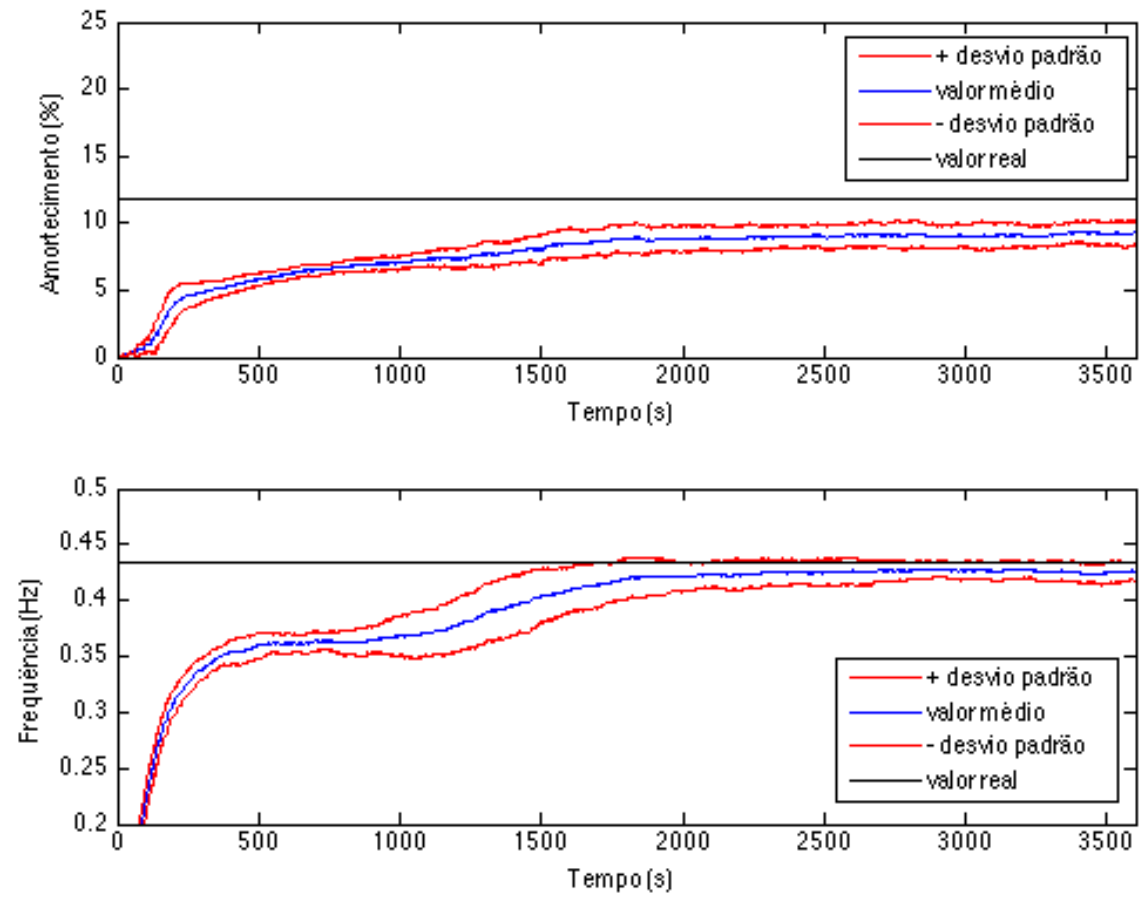

Figura 4.10: Frequência e amortecimento do modo de oscilação identificado no quarto caso utilizando modelagem com dois modos de oscilação

trabalhos relatam que a obtenção de valores para o amortecimento dos modos identificados é mais difícil do que para a frequência (ver por exemplo [2]).

\subsubsection{Quinto caso - um modo dominante fixo}

O quinto e último caso simulado possui o modo de oscilação fixo descrito na Tabela 4.5. Trata-se do modo de oscilação entre as duas áreas do sistema teste, mas com amortecimento inferior em relação ao amortecimento simulado no quarto caso. Para tanto, os valores de ganho dos PSSs dos geradores síncronos do sistema teste foram alterados, além das alterações realizadas nas constantes de inércia dos geradores para o quarto e quinto casos.

Tabela 4.5: Modo de oscilação constante do quinto caso.

\begin{tabular}{c|c}
\hline Frequência $(\mathrm{Hz})$ & Amortecimento $(\%)$ \\
\hline 0,4438 & 3,072 \\
\hline
\end{tabular}

Inicialmente, a algoritmo de FKE foi aplicado utilizando um modelo constituído de apenas um modo. A Figura 4.11 apresenta a média dos valores encontrados para frequência e amortecimento do modo de oscilação ao longo do período de uma hora de simulação, considerando as 100 simulações realizadas, bem como o desvio padrão 
desses valores. O padrão de cores desta figura é o mesmo daquele adotado para todos os casos.
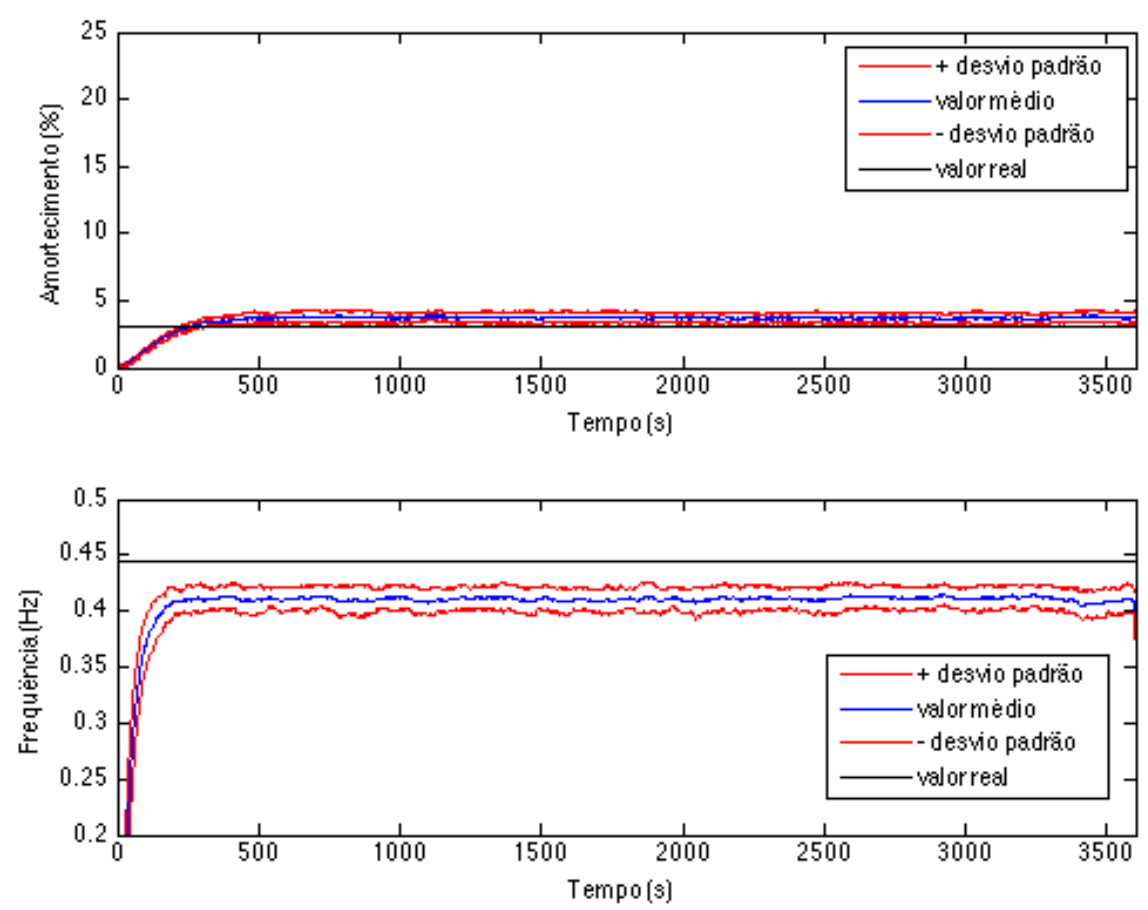

Figura 4.11: Frequência e amortecimento do modo de oscilação identificado no quinto caso utilizando modelagem com um modo de oscilação

Assim como no quarto caso, observa-se na Figura 4.11 que, apesar da convergência do algoritmo, os valores encontrados para frequência do modo de oscilação não estão coerentes com os valores reais. Ao analisar o espectro de frequências deste sinal, considerando as 100 simulações realizadas, como pode ser visto na Figura 4.12, percebe-se que a frequência do modo identificado é bem diferente da frequência da componente do pico de magnitude no espectro de frequências.

O algoritmo de FKE foi então aplicado utilizando a modelagem com dois modos de oscilação. A Figura 4.13 apresenta o espectro de frequências do sinal, considerando as 100 simulações realizadas, com destaque nas frequências dos dois modos de oscilação identificados.

Percebe-se pela Figura 4.13 que o modo de oscilação com maior frequência identificado está coerente com o pico de magnitude do espectro de frequências do sinal. A Figura 4.14 apresenta os valores de frequência e amortecimento identificados associados a este modo de oscilação de maior frequência.

Como pode ser visto na Figura 4.14, utilizando a modelagem com dois modos de 


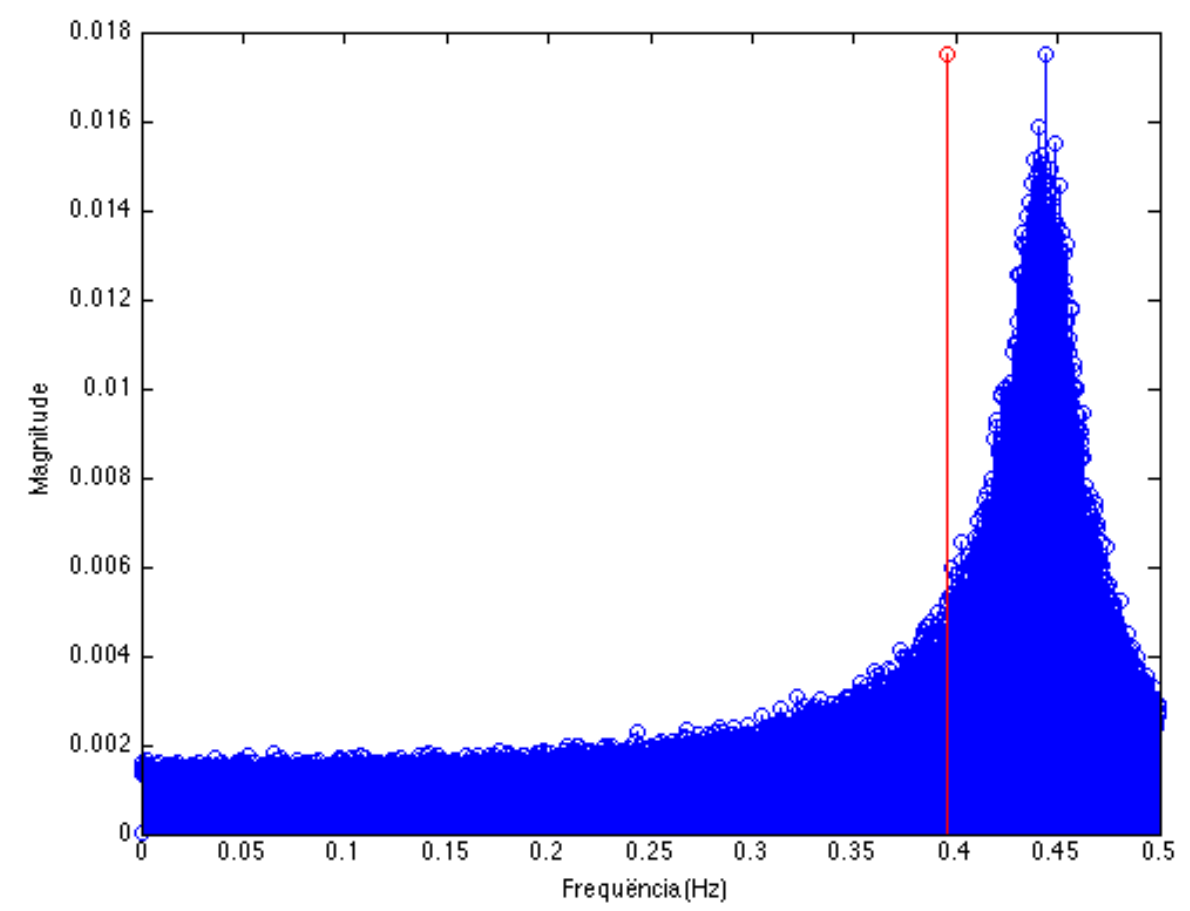

Figura 4.12: Espectro de frequências do sinal simulado no quinto caso utilizando um modelo com um modo com destaque para a frequência do modo de oscilação identificado

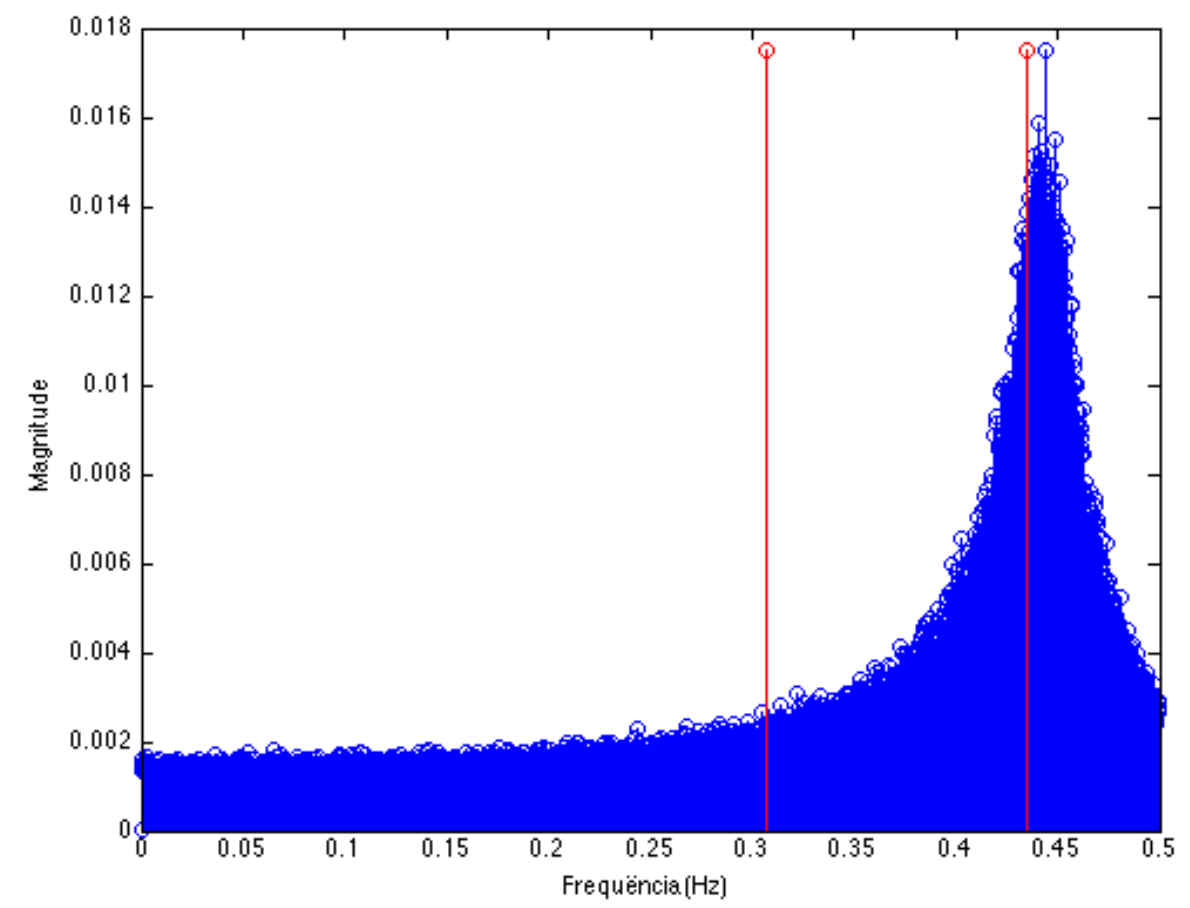

Figura 4.13: Espectro de frequências do sinal simulado no quinto caso utilizando um modelo com dois modos com destaque para a frequência dos dois modos de oscilação identificados

oscilação, os valores de frequência e amortecimento do modo de oscilação identificado são coerentes com os valores reais. Os valores de amortecimento identificados são ligeiramente superiores aos valores reais, enquanto os de frequência são ligeiramente 

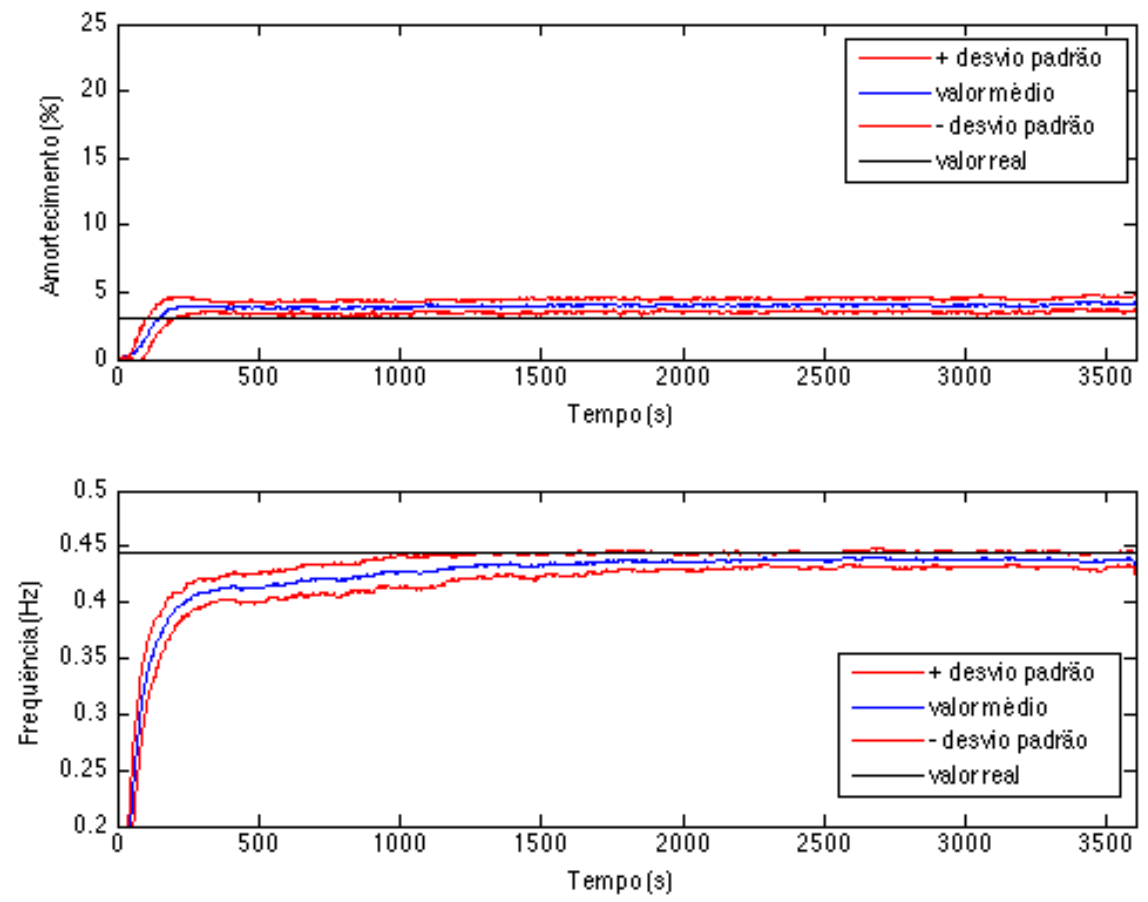

Figura 4.14: Frequência e amortecimento do modo de oscilação identificado no quinto caso utilizando modelagem com dois modos de oscilação

inferiores.

Neste caso, também foi aplicado o algoritmo de FKE utilizando a modelagem com três modos de oscilação para que fosse feita uma comparação com o resultado quando se utiliza apenas dois modos. A Figura 4.15 ilustra o espectro de frequências do sinal, considerando as 100 simulações realizadas, com os três modos encontrados em destaque.

Observa-se que os dois modos de maior frequência identificados possuem frequências próximas uma da outra e ambas coerentes com o pico de magnitude do espectro de frequências do sinal. As Figuras 4.16 e 4.17 apresentam as frequências e amortecimentos identificados para esses dois modos.

Analisando as Figuras 4.16 e 4.17, fica claro que os resultados para esses dois modos são praticamente iguais, e ambos são praticamente iguais àquele da Figura 4.14, quando a modelagem possuía apenas dois modos de oscilação. Conclui-se que a modelagem com três polos identificou polos praticamente sobrepostos, ambos com frequências próximas ao pico de magnitude do espectro de frequências, associado ao modo de oscilação do sinal. Portanto, neste caso, o ideal é a modelagem com apenas dois modos de oscilação, por ser mais simples e para que não haja sobreposição de modos identificados nem o surgimento de modos espúrios. 


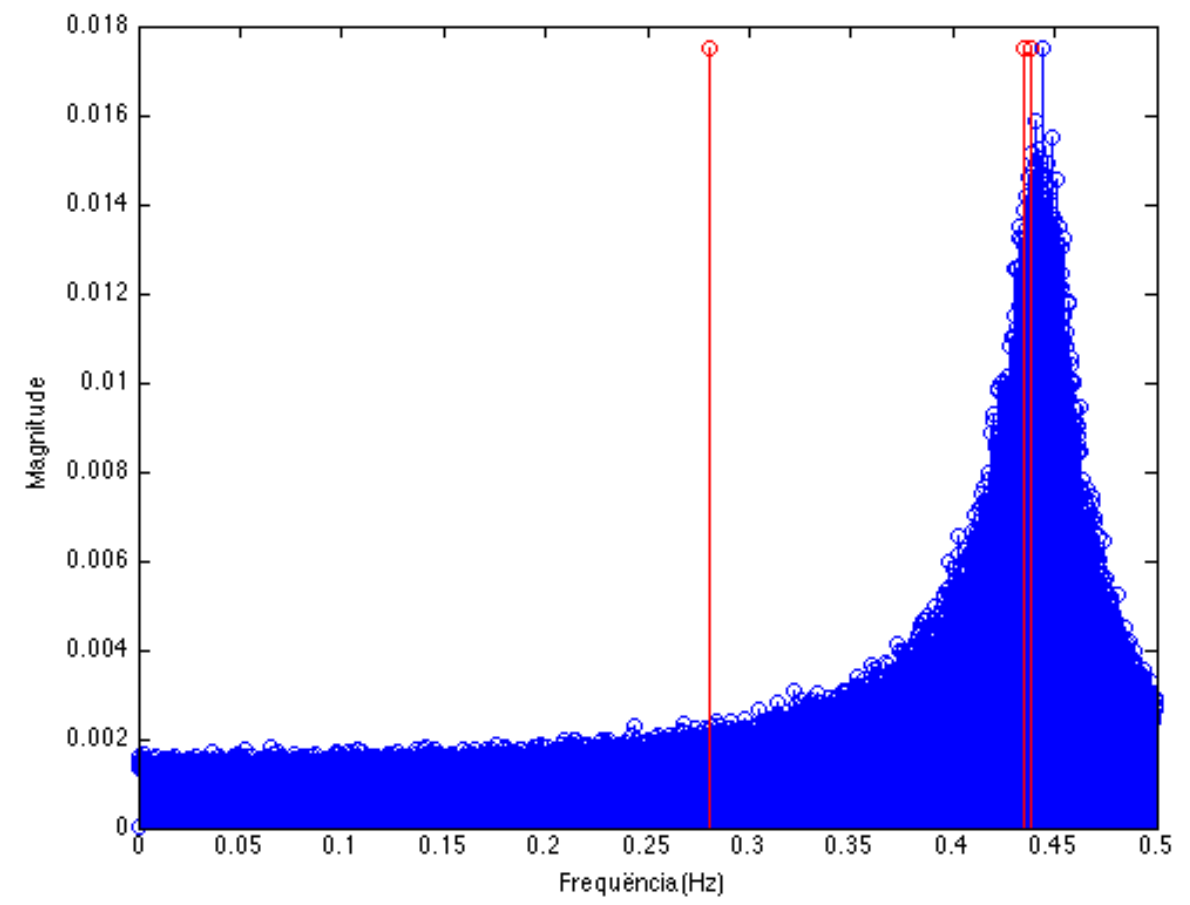

Figura 4.15: Espectro de frequências do sinal simulado no quinto caso utilizando um modelo com três modos com destaque para a frequência dos três modos de oscilação identificados
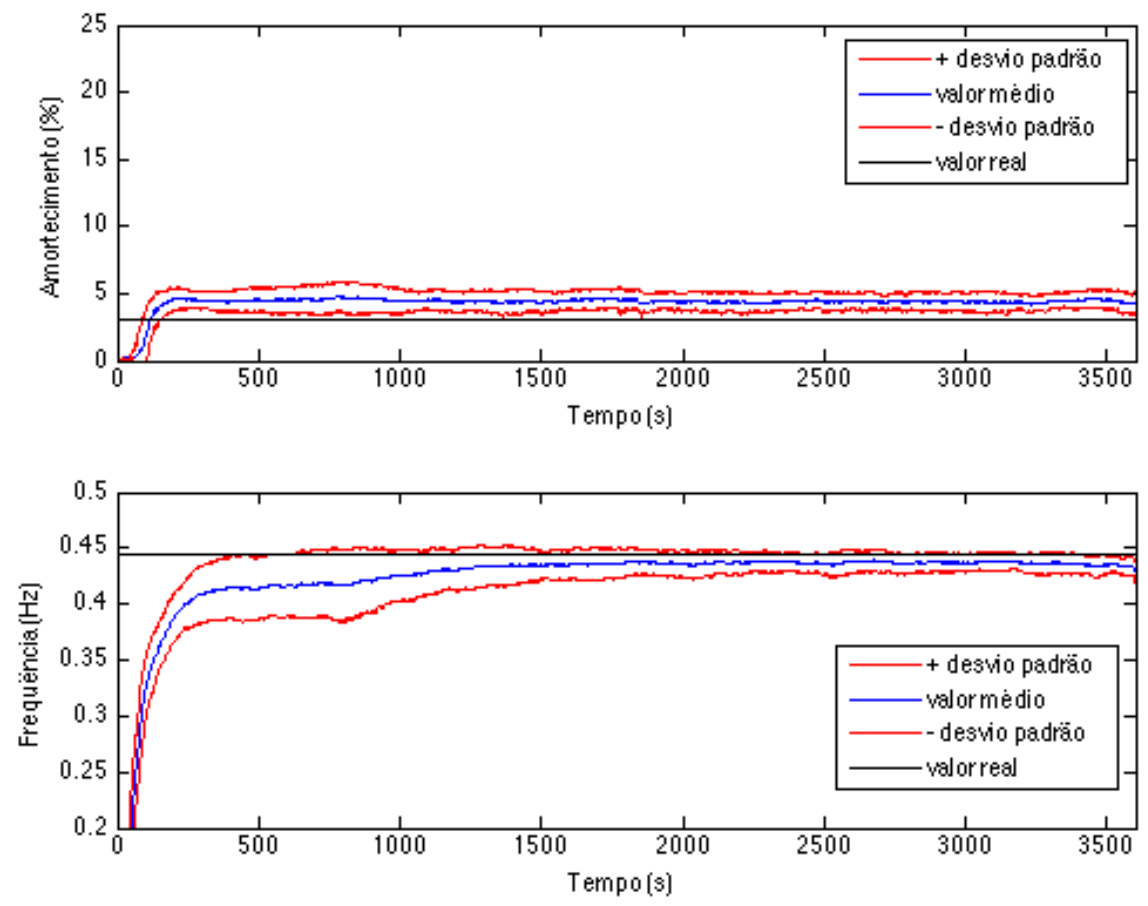

Figura 4.16: Frequência e amortecimento de um dos modos de oscilação identificados no quinto caso utilizando modelagem com três modos de oscilação

\subsection{Aplicações em sinais de PMUs do SIN}

Nesta seção descrevem-se resultados da aplicação do FKE em dados de ambiente provenientes de PMUs, conforme metodologia descrita no Capítulo 3. Os cálculos são emba- 

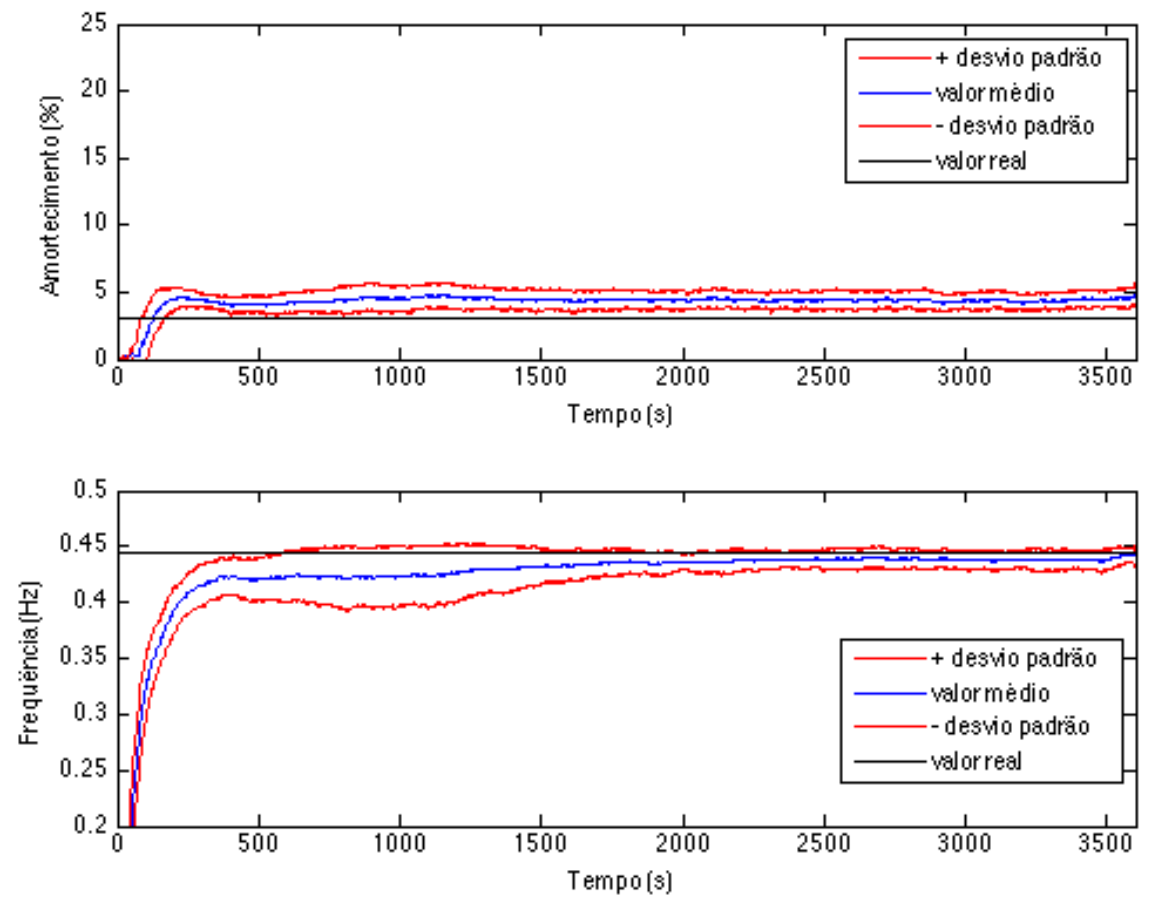

Figura 4.17: Frequência e amortecimento de um dos modos de oscilação identificados no quinto caso utilizando modelagem com três modos de oscilação

sados na utilização de dados de campo obtidos de algumas PMUs do projeto Medfasee [10]. O objetivo é observar o comportamento do modo de oscilação eletromecânica interárea Norte-Sul do SIN. A análise foi efetuada em uma longa janela de dados em que as perturbações são devidas ao próprio ruído ambiente do sistema elétrico. Com esta finalidade, são calculados (estimados) o amortecimento e a frequência do modo de oscilação mais dominante do SIN.

O FKE foi utilizado com as seguintes matrizes de covariâncias $P_{0}=I_{4 p}, \overline{Q_{X}}=100 I_{2 p}$, $Q_{P a}=20, Q_{P b}=0,1, R=1$, em que $I_{j}$ é a matriz identidade de ordem $j, p$ é o número de modos a serem estimados pelo modelo e $Q_{P a}$ e $Q_{P b}$ são os elementos da matriz de covariâncias $\overline{Q_{P}}$ associados aos parâmetros $a_{k}$ e $b_{k}$, respectivamente. Todos os estados $x_{0}$, incluindo aqueles representativos dos parâmetros utilizados para calcular os modos de oscilação, foram inicializados com valores iguais a zero. Nenhum problema foi identificado com relação à convergência final de parâmetros.

Os dados utilizados neste trabalho referem-se a informações de ângulo de tensão coletados no dia 14 de outubro de 2014, no período de 20h00 às 21h00 (janela com 1 hora de dados), hora local de Brasília. Os dados foram obtidos à frequência de 60 amostras/s. Na janela de dados em questão, nenhuma ocorrência em termos de perturbação na rede elétrica, merecedora de destaque, foi registrada nos boletins de divulgação do 
Operador Nacional do Sistema Elétrico (ONS) [81].

As informações foram obtidas das PMUs localizadas na UFC, UFT, UFRGS, UFSC, UNIPAMPA, UTFPR e UFMA. Embora nos testes tenham sido utilizados dados para simulações com ângulos das tensões das três fases da rede trifásica, somente são mostrados os resultados relativos à fase referenciada como $\mathrm{A}$.

O algoritmo de FKE foi utilizado de modo a permitir a estimação dos parâmetros que caracterizam os polos complexos. Com base nestes parâmetros, obtêm-se os valores instantâneos de amortecimento e de frequência que caracterizam os modos de oscilação do modelo identificado. Os parâmetros modais são estimados a cada amostra do sinal.

A Tabela 4.6 ilustra faixas típicas de valores de frequências associadas a modos de oscilação interárea no SIN [9]. O interesse é por valores na faixa relativa à primeira linha da tabela, portanto, entre $0,20 \mathrm{~Hz}$ e $0,40 \mathrm{~Hz}$.

Tabela 4.6: Faixas de valores de frequência de modos de oscilação interárea conhecidos do SIN [9].

\begin{tabular}{c|c}
\hline Modo & Faixa de frequência \\
\hline Norte x Sul & $0,20-0,40 \mathrm{~Hz}$ \\
Sul x Sudeste & $0,60-0,80 \mathrm{~Hz}$ \\
Norte x Nordeste & $0,55-0,65 \mathrm{~Hz}$ \\
Mato Grosso x SIN & $0,40-0,45 \mathrm{~Hz}$ \\
Rio de Janeiro x SIN & $1,10-1,30 \mathrm{~Hz}$ \\
São Paulo x SIN & $0,65-0,75 \mathrm{~Hz}$ \\
\hline
\end{tabular}

A observação do amortecimento e da frequência do modo Norte-Sul foi realizada considerando-se sinais de diferença angular (ângulos relativos). Com esta finalidade, foram feitos estudos para se determinar a melhor referência angular considerando os sinais de ângulo medido nas PMUs da UFT, da UFMA e da UFC (regiões Norte e Nordeste do Brasil). A escolha dos sinais de PMU a serem analisados consiste em uma das dificuldades associadas à identificação de modos de oscilação, pois o ideal é analisar sinais com alta observabilidade dos modos de interesse e baixa dos outros $[2,34]$.

Para cada um dos sinais de diferença angular obtidos, o FKE foi aplicado utilizando-se modelos com um, dois, três e quatro modos de oscilação. Aqui estão apresentados apenas os resultados da aplicação dos modelos com dois e três modos, suficientes para 
entendimento do processo. Foram estimados os polos para essas duas situações de modelo e determinados os espectros de frequência de cada sinal.

\subsubsection{Sinais com referência na UFC}

Nesta seção são apresentados os resultados de aplicação do algoritmo de FKE aos sinais de diferença angular de tensão das PMUs instaladas na UFSC, UFRGS, UNIPAMPA e UTFPR, todas no Sul do Brasil, tendo como referência o ângulo de tensão da fase A da PMU instalada na UFC, no Nordeste. Espera-se, com a escolha desses sinais, obter uma boa observabilidade do modo de oscilação Norte-Sul, na faixa entre $0,2 \mathrm{~Hz}$ e 0,4 $\mathrm{Hz}$.

A Figura 4.18 apresenta os espectros de frequências desses sinais após o pré-processamento descrito na Seção 3.3. Em vermelho está destacado o espectro do sinal antes da filtragem descrita na Seção 3.3.3, e em azul o espectro após essa filtragem.
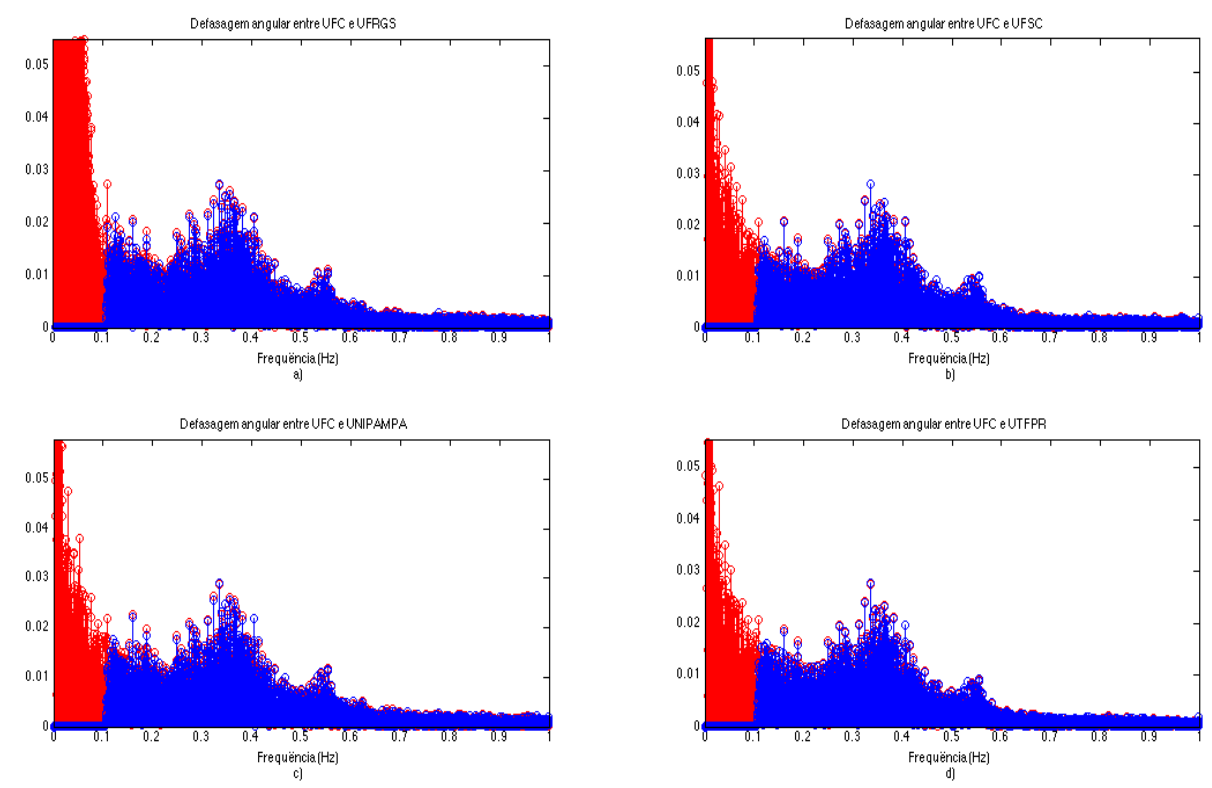

Figura 4.18: Espectro de frequências dos sinais: a) defasagem angular de tensão entre a PMU da UFC e a da UFRGS; b) entre a PMU da UFC e a da UFSC; c) entre a PMU da UFC e a da UNIPAMPA e d) entre a PMU da UFC e a da UTFPR.

Observa-se que os quatro gráficos de espectro de frequências apresentam picos de magnitude entre as frequências $0,3 \mathrm{~Hz}$ e $0,4 \mathrm{~Hz}$, indicando a presença de um modo de oscilação na faixa de frequências do modo Norte-Sul. Outro pico pode ser observado também entre $0,5 \mathrm{~Hz}$ e 0,6 Hz, no entanto, apesar de tratar-se de faixa de frequência associada a modo de oscilação interárea, não foi objeto de estudo neste trabalho, pois apresenta menor dominância quando comparado ao modo Norte-Sul. 
O espectro de frequências antes da filtragem descrita na Seção 3.3.3 é apresentado na Figura 4.18 com o intuito de deixar claro que entre as frequências $0,1 \mathrm{~Hz}$ e $0,2 \mathrm{~Hz}$ dos sinais não há um pico de magnitude ocasionado por um modo de oscilação, mas sim pela frequência de corte do filtro passa-alta utilizado no pré-processamento dos sinais.

O algoritmo de FKE proposto neste trabalho foi aplicado aos sinais de PMU de defasagem angular de tensão da UFRGS, UFSC, UNIPAMPA e UTFPR em relação a da UFC, utilizando a modelagem com dois e três modos de oscilação.

As Figuras 4.19 e 4.20 apresentam os espectros desses sinais com destaque em vermelho para as frequências dos modos de oscilação encontrados quando utilizada a modelagem com dois e três modos, respectivamente.
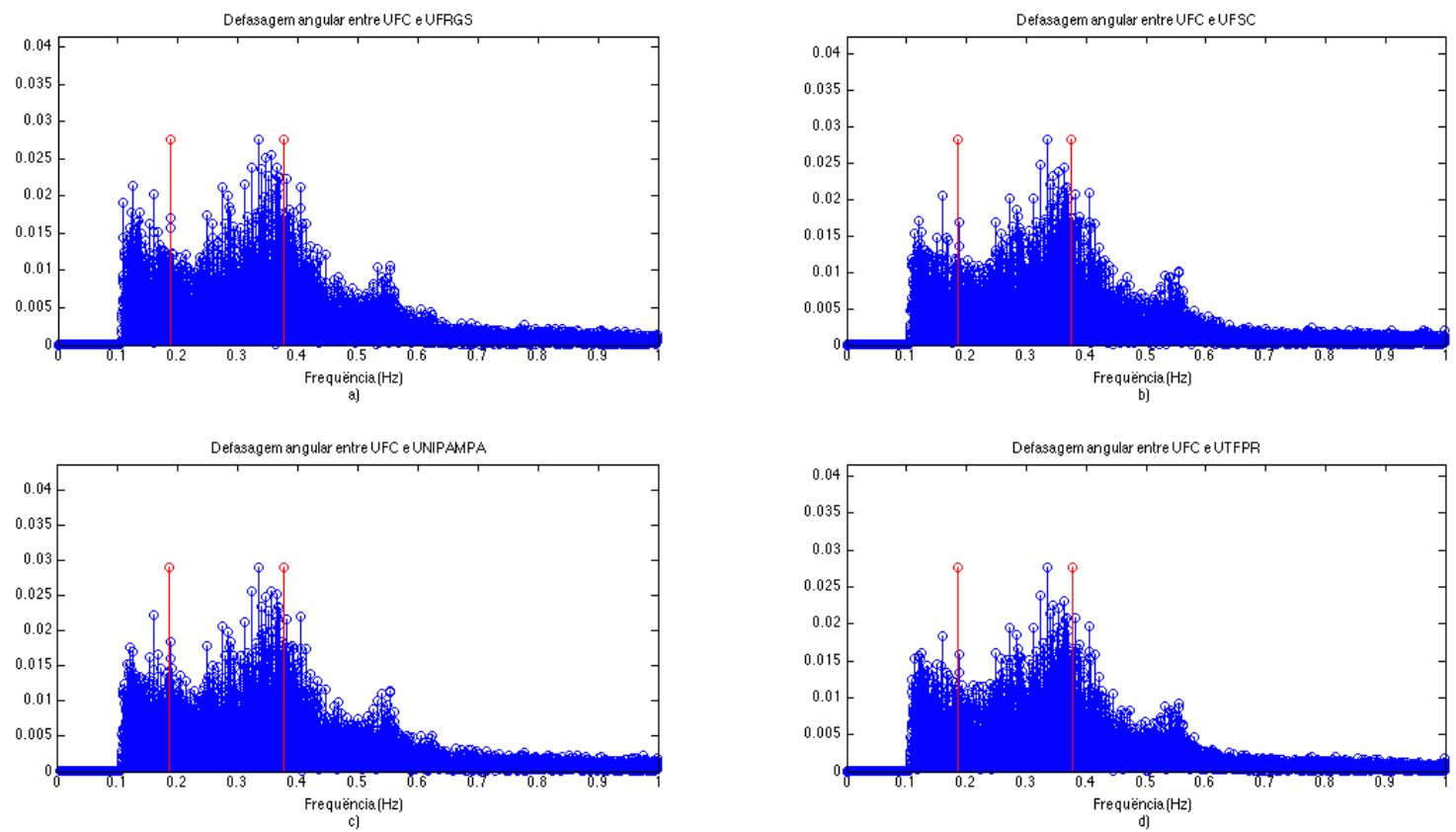

Figura 4.19: Espectro de frequências, com destaque em vermelho para as frequências dos dois modos identificados, dos sinais: a) defasagem angular de tensão entre a PMU da UFC e a da UFRGS; b) entre a PMU da UFC e a da UFSC; c) entre a PMU da UFC e a da UNIPAMPA e d) entre a PMU da UFC e a da UTFPR.

As duas modelagens encontraram modos de oscilação com frequência entre $0,2 \mathrm{~Hz}$ e 0,4 $\mathrm{Hz}$, característica do modo Norte-Sul. Observa-se, no entanto, que a modelagem com dois modos de oscilação, para todos os sinais, encontrou um modo com frequência mais próxima do pico de magnitude de cada espectro. A Figura 4.21 apresenta os valores de amortecimento e frequência para o modo de oscilação encontrado para esses sinais utilizando a modelagem com dois modos.

Verifica-se que, após o período de convergência do algoritmo, aproximadamente 1000 segundos de sinal, o comportamento do modo de oscilação em todos os sinais é o 

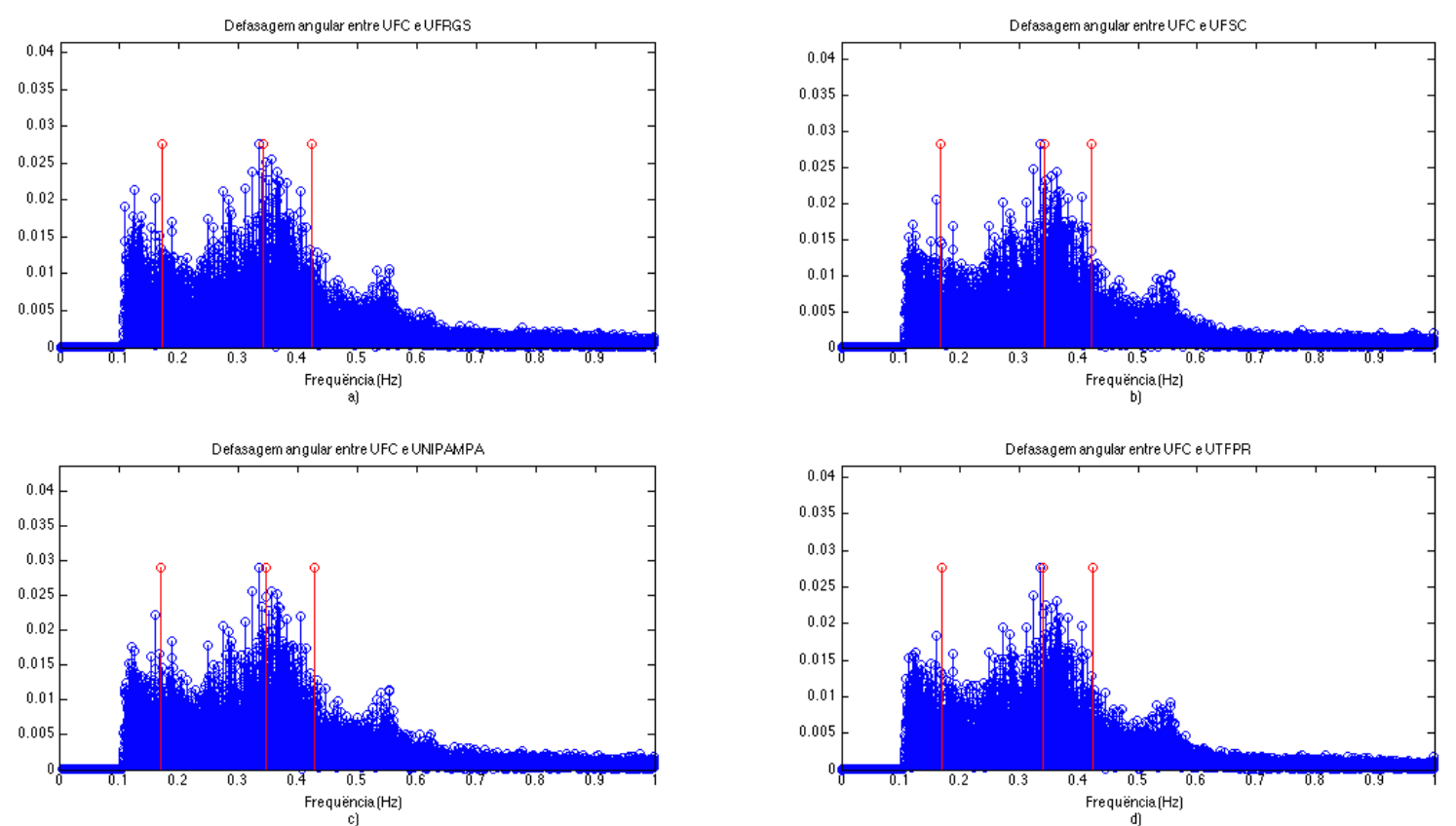

Figura 4.20: Espectro de frequências, com destaque em vermelho para as frequências dos três modos identificados, dos sinais: a) defasagem angular de tensão entre a PMU da UFC e a da UFRGS; b) entre a PMU da UFC e a da UFSC; c) entre a PMU da UFC e a da UNIPAMPA e d) entre a PMU da UFC e a da UTFPR.
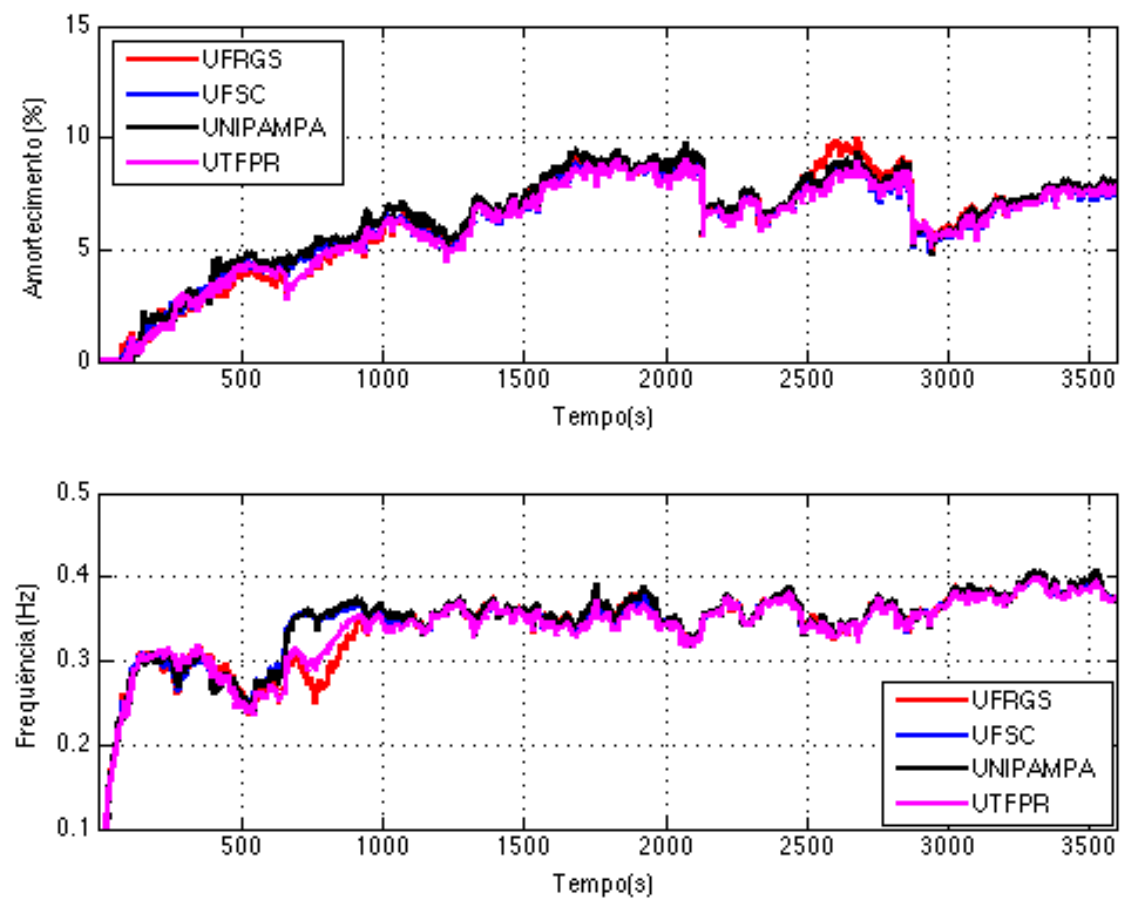

Figura 4.21: Amortecimento e frequência do modo de oscilação identificado nos sinais de defasagem angular de tensão entre as PMUs da UFRGS, UFSC, UNIPAMPA e UTFPR em relação à da UFC, utilizando modelagem com dois modos

mesmo, o que destaca a observabilidade do modo Norte-Sul nestes sinais. Os valores encontrados para a frequência do modo variam entre $0,3 \mathrm{~Hz}$ e $0,4 \mathrm{~Hz}$, e para amortecimento variam entre $5 \%$ e $10 \%$. A Tabela 4.7 apresenta as médias dos valores de 
amortecimento e frequência para os últimos 10 minutos de cada sinal.

Tabela 4.7: Modo de oscilação identificado nos sinais com referência na PMU da UFC

\begin{tabular}{c|c|c}
\hline Sinal & Frequência $(\mathrm{Hz})$ & Amortecimento $(\%)$ \\
\hline UFRGS & 0,3848 & 7,2362 \\
UFSC & 0,3830 & 6,9965 \\
UNIPAMPA & 0,3867 & 7,2692 \\
UTFPR & 0,3808 & 7,0364 \\
\hline
\end{tabular}

O acompanhamento da dinâmica de um sistema elétrico deve ser contínuo. Desta forma, um algoritmo que se proponha a identificar o modo de oscilação dominante do sistema em tempo real será iniciado apenas uma vez e os parâmetros a serem identificados irão convergir para os valores estimados apenas na inicialização. Como forma de simular a identificação em tempo real do modo dominante do sistema, o algoritmo de FKE foi aplicado aos sinais analisados nesta seção utilizando como valores iniciais dos estados $\hat{x}_{0}$ aqueles associados aos valores de frequência e amortecimento da Tabela 4.7. Desta forma eliminou-se o período de convergência do algoritmo. O resultado pode ser visto na Figura 4.22.
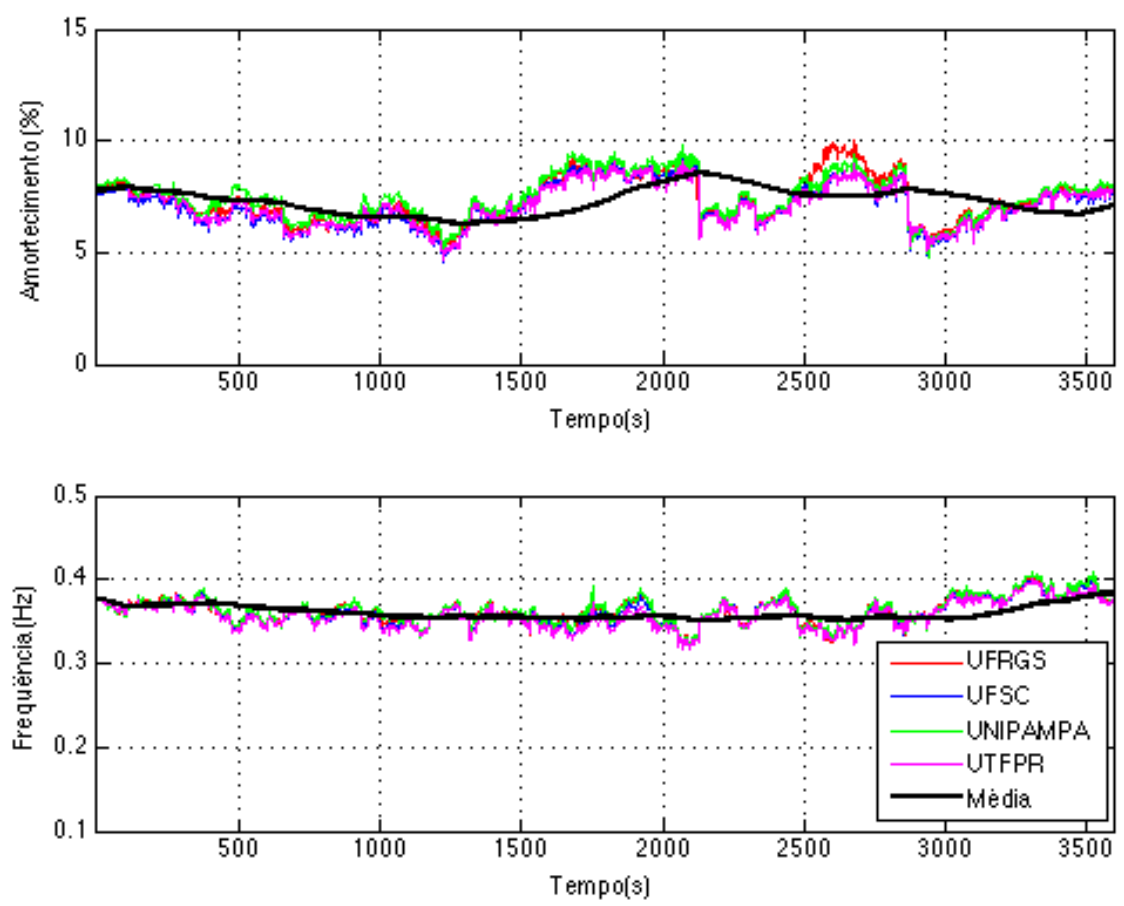

Figura 4.22: Média móvel de 10 minutos do amortecimento e da frequência do modo de oscilação identificado nos sinais de defasagem angular de tensão

Nesta figura pode ser observado como seria o comportamento do algoritmo em operação 
em tempo real. A curva destacada na cor preta é a média móvel de 10 minutos dos valores de amortecimento e frequência para os quatro sinais analisados.

A Figura 4.23 ilustra uma comparação entre os modos de oscilação encontrados utilizando a modelagem com dois e três modos, na faixa de 0,2 a $0,4 \mathrm{~Hz}$. Observa-se que os valores de frequência e amortecimento encontrados utilizando a modelagem com três modos são ligeiramente menores, mas o comportamento dos valores é o mesmo para ambas as modelagens.
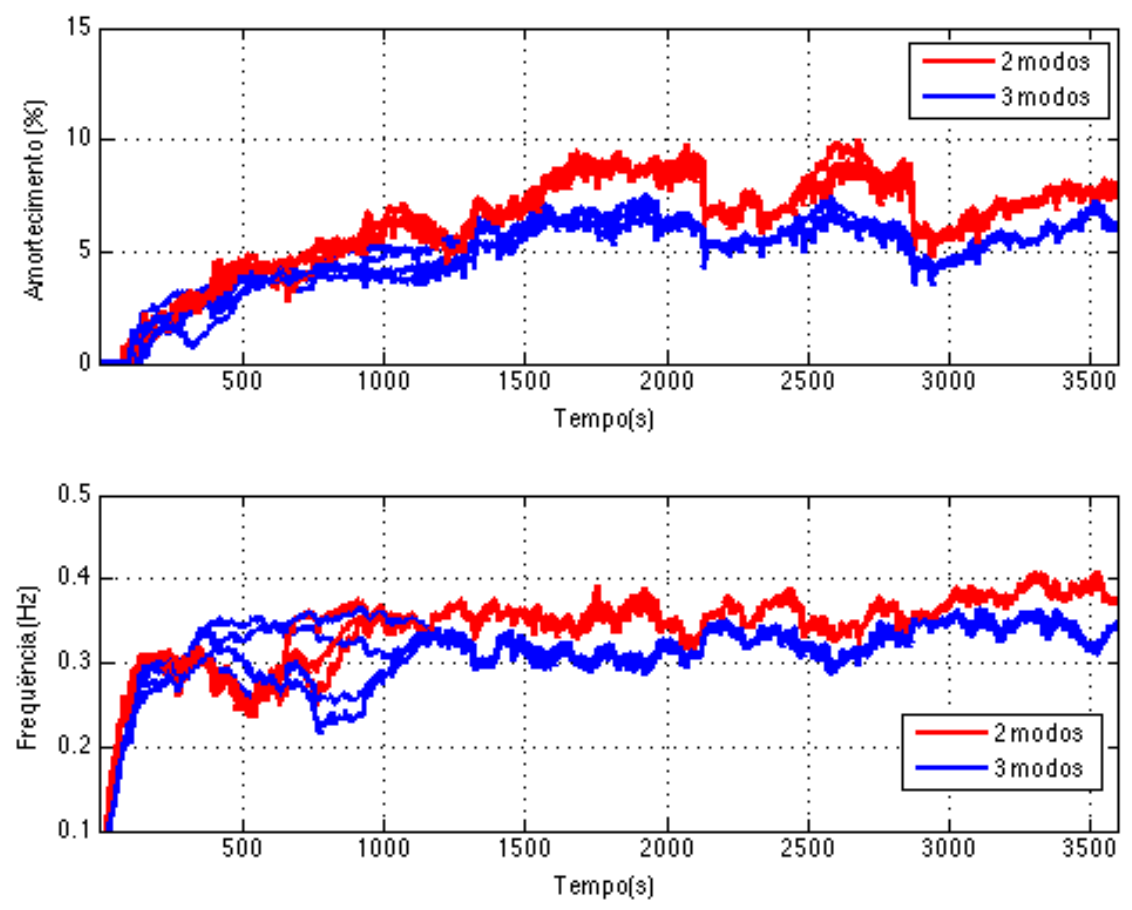

Figura 4.23: Comparação dos valores de amortecimento e frequência do modo de oscilação identificado utilizando modelagem com dois e três modos

\subsubsection{Sinais com referência na UFT}

Nesta seção são apresentados os resultados de aplicação do algoritmo de FKE aos sinais de diferença angular de tensão das PMUs instaladas na UFSC, UFRGS, UNIPAMPA e UTFPR tendo como referência o ângulo de tensão da fase A da PMU instalada na UFT, no Norte, da mesma forma como foi feito para os sinais com referência na PMU da UFC na seção 4.3.1.

A Figura 4.24 apresenta os espectros de frequências desses sinais após o pré-processamento descrito na Seção 3.3. Em vermelho está destacado o espectro do sinal antes da filtragem descrita na Seção 3.3.3, e em azul o espectro após essa filtragem. 

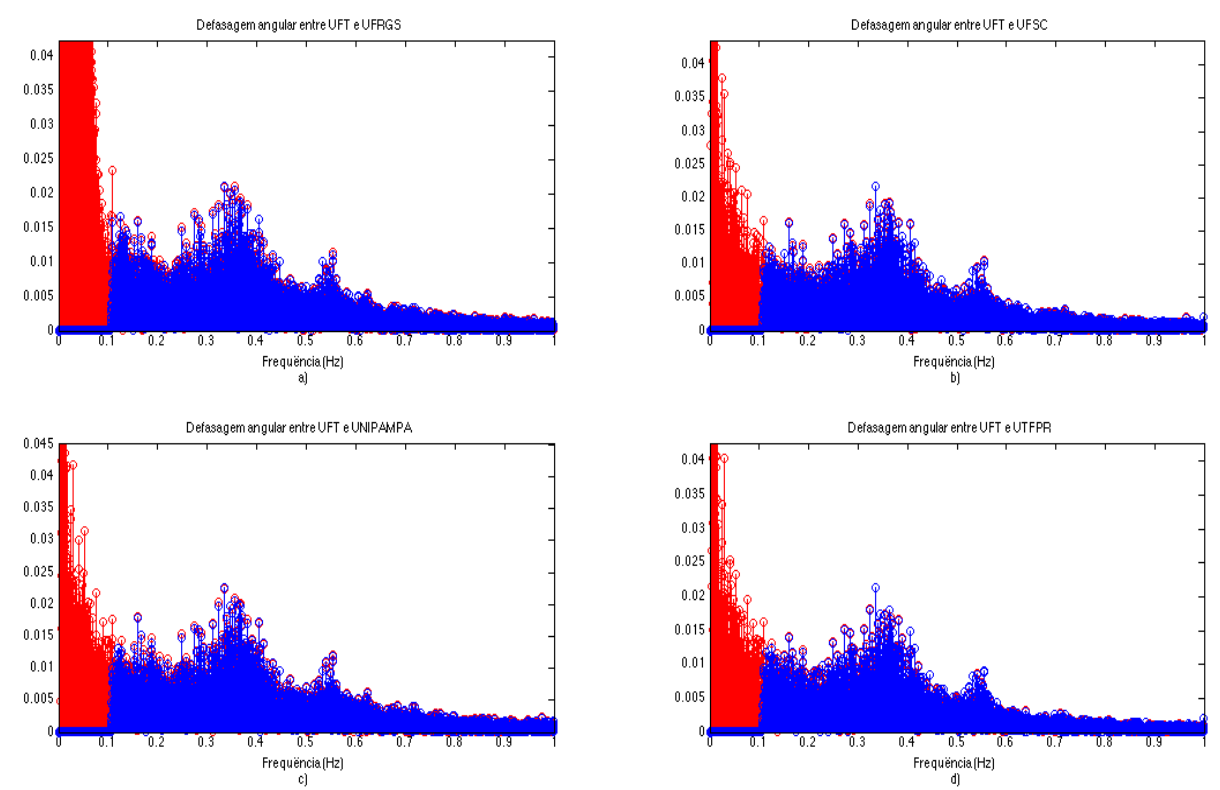

Figura 4.24: Espectro de frequências dos sinais: a) defasagem angular de tensão entre a PMU da UFT e a da UFRGS; b) entre a PMU da UFT e a da UFSC; c) entre a PMU da UFT e a da UNIPAMPA e d) entre a PMU da UFT e a da UTFPR.

Observa-se que os quatro gráficos de espectro de frequências apresentam picos de magnitude entre as frequências $0,3 \mathrm{~Hz}$ e $0,4 \mathrm{~Hz}$, indicando a presença de um modo de oscilação na faixa de frequências do modo Norte-Sul.

As Figuras 4.25 e 4.26 apresentam os espectros desses sinais com destaque em vermelho para as frequências dos modos de oscilação encontrados após a aplicação do algoritmo de FKE quando utilizada a modelagem com dois e três modos, respectivamente.

As duas modelagens encontraram modos de oscilação com frequência entre $0,2 \mathrm{~Hz}$ e 0,4 $\mathrm{Hz}$, característica do modo Norte-Sul. Observa-se, no entanto, que a modelagem com três modos de oscilação, para todos os sinais, encontrou um modo com frequência mais próxima do pico de magnitude de cada espectro. A Figura 4.27 apresenta os valores de amortecimento e frequência para o modo de oscilação encontrado para esses sinais utilizando a modelagem com três modos.

Verifica-se que, após o período de convergência do algoritmo, aproximadamente 1500 segundos de sinal, o comportamento do modo de oscilação em todos os sinais é o mesmo. Os valores encontrados para a frequência do modo variam entre $0,3 \mathrm{~Hz}$ e $0,4 \mathrm{~Hz}$, e para amortecimento variam entre $4 \%$ e $9 \%$. A Tabela 4.8 apresenta a média dos valores de amortecimento e frequência para os últimos 10 minutos de sinal. 

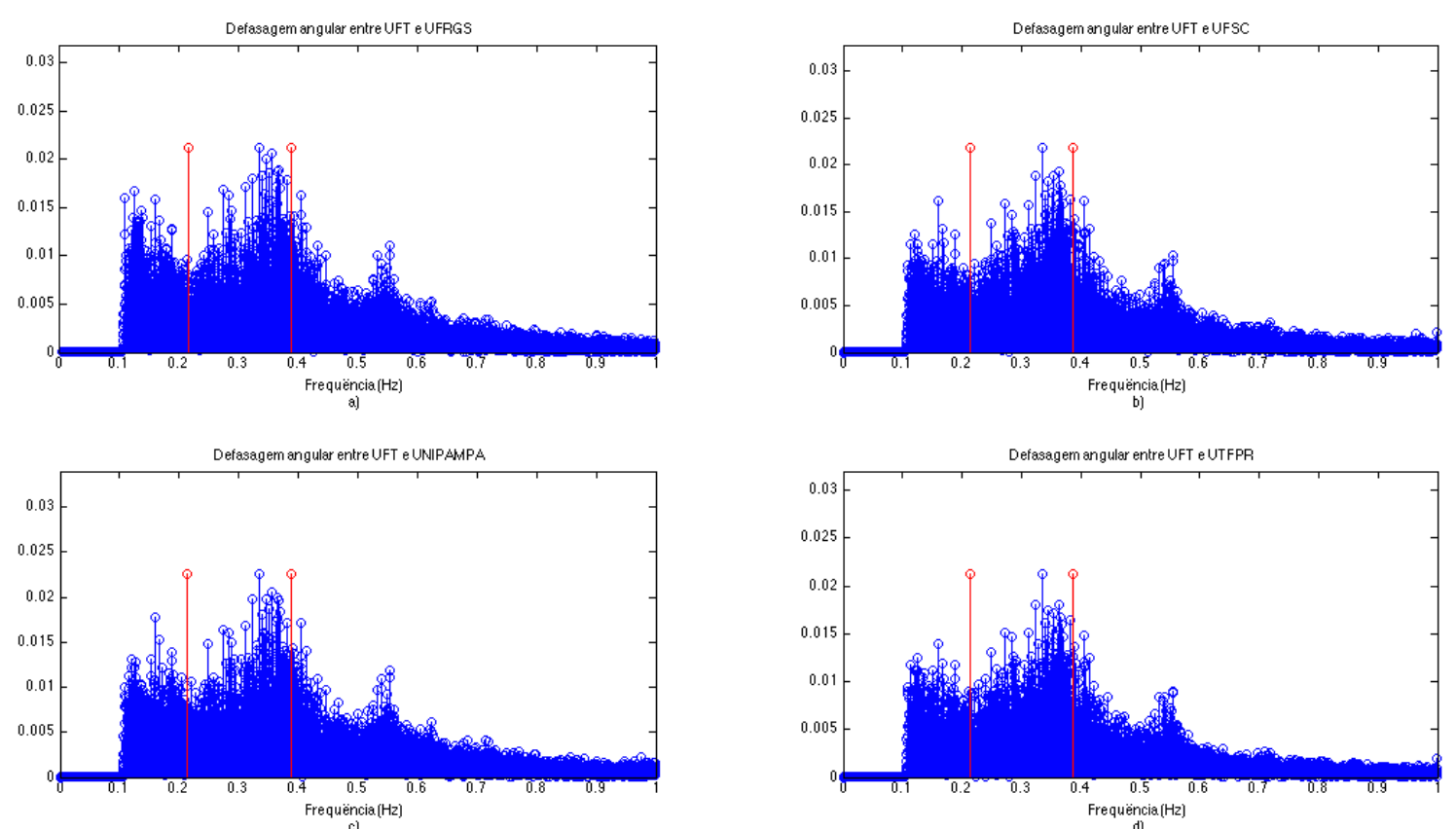

Figura 4.25: Espectro de frequências, com destaque em vermelho para as frequências dos dois modos identificados, dos sinais: a) defasagem angular de tensão entre a PMU da UFT e a da UFRGS; b) entre a PMU da UFT e a da UFSC; c) entre a PMU da UFT e a da UNIPAMPA e d) entre a PMU da UFT e a da UTFPR.
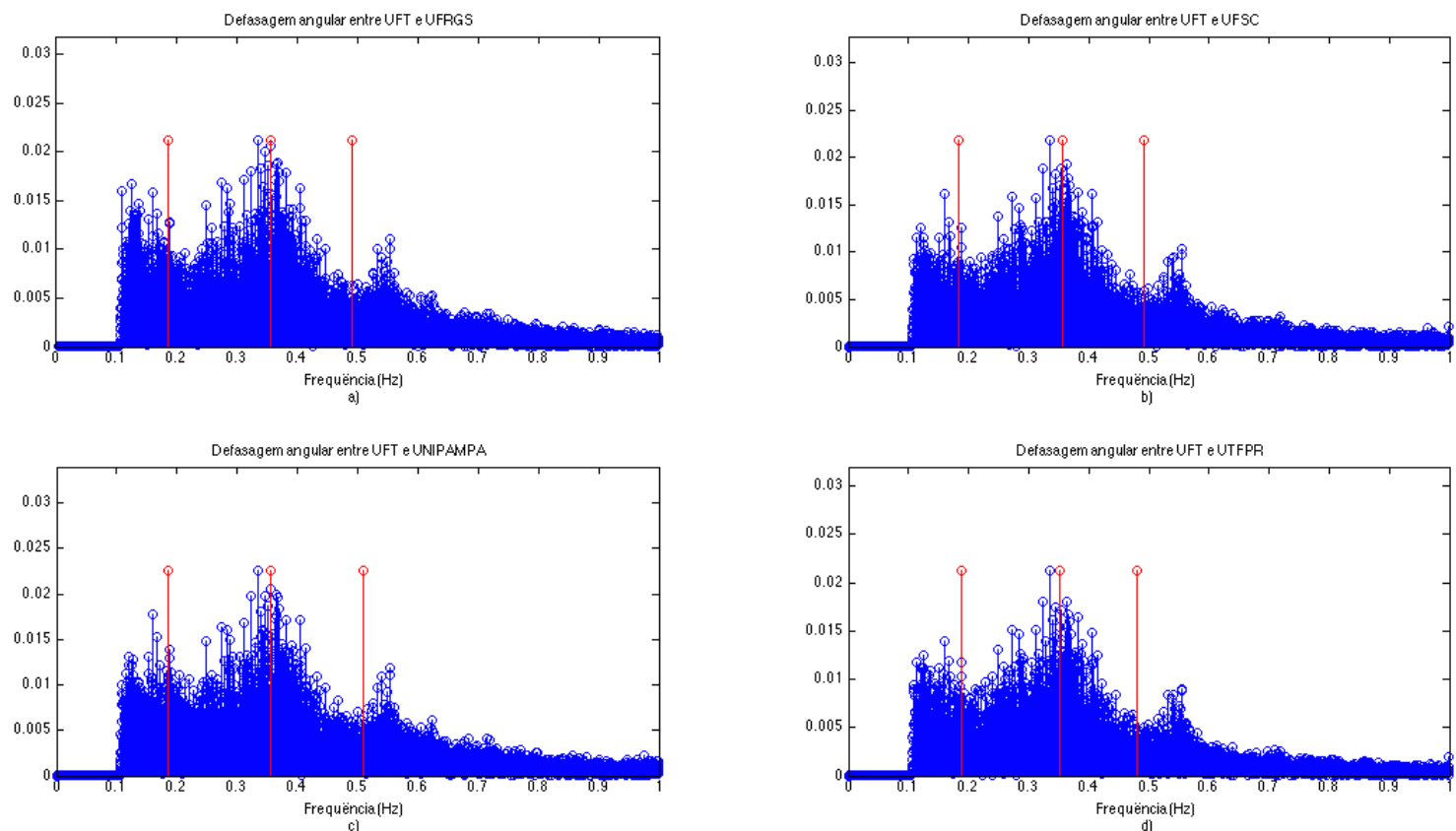

Figura 4.26: Espectro de frequências, com destaque em vermelho para as frequências dos três modos identificados, dos sinais: a) defasagem angular de tensão entre a PMU da UFT e a da UFRGS; b) entre a PMU da UFT e a da UFSC; c) entre a PMU da UFT e a da UNIPAMPA e d) entre a PMU da UFT e a da UTFPR.

Assim como foram analisados os sinais com referência na UFC, o algoritmo de FKE foi aplicado aos sinais analisados nesta seção utilizando como valores iniciais dos estados $x_{0}$ aqueles associados aos valores de frequência e amortecimento da Tabela 4.8. O resultado pode ser visto na Figura 4.28. 

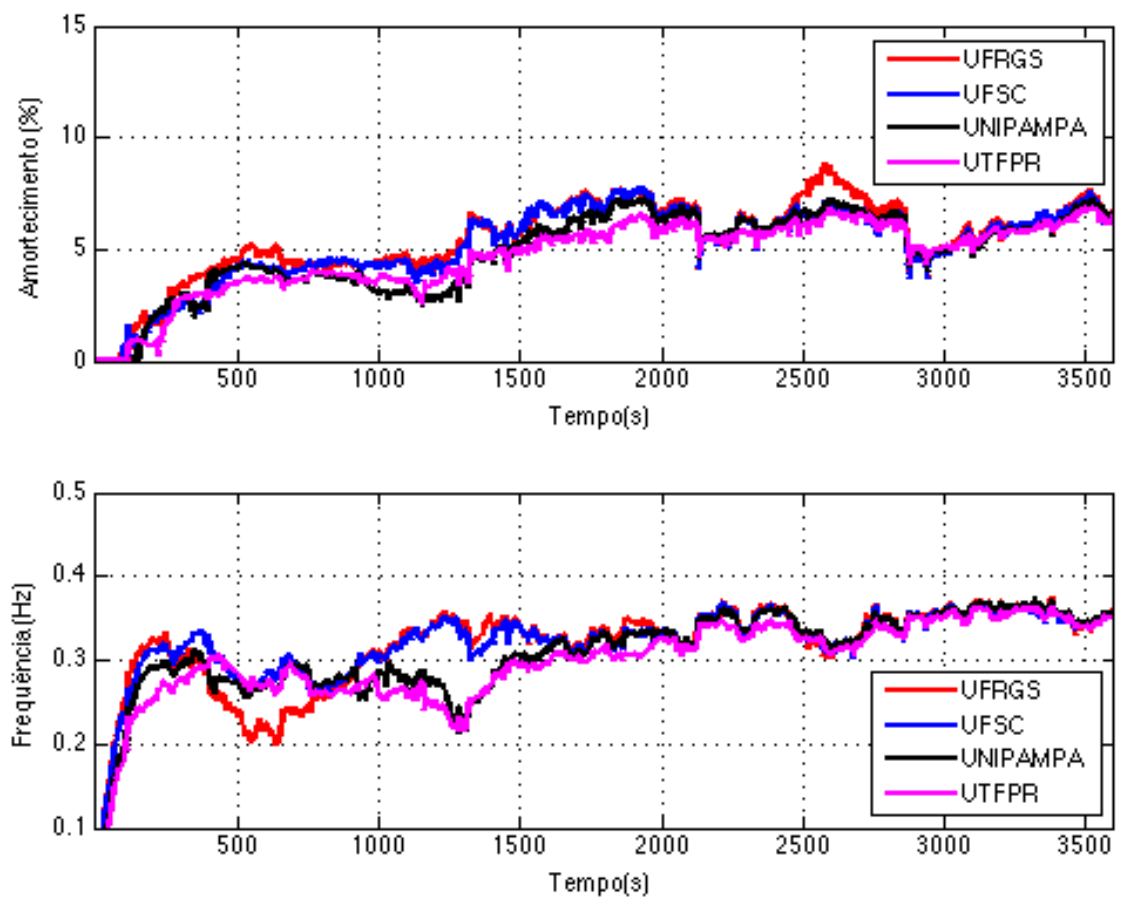

Figura 4.27: Amortecimento e frequência do modo de oscilação identificado nos sinais de defasagem angular de tensão entre as PMUs da UFRGS, UFSC, UNIPAMPA e UTFPR em relação à da UFT, utilizando modelagem com três modos

Tabela 4.8: Modo de oscilação identificado nos sinais com referência na PMU da UFT

\begin{tabular}{c|c|c}
\hline Sinal & Frequência $(\mathrm{Hz})$ & Amortecimento $(\%)$ \\
\hline UFRGS & 0,3569 & 6,2550 \\
UFSC & 0,3557 & 6,1527 \\
UNIPAMPA & 0,3585 & 5,9861 \\
UTFPR & 0,3532 & 6,0216 \\
\hline
\end{tabular}

Nesta Figura pode ser observado como seria o comportamento do algoritmo em operação em tempo real. A curva destacada na cor preta é a média móvel de 10 minutos dos valores de amortecimento e frequência para os quatro sinais analisados.

A Figura 4.29 ilustra uma comparação entre os modos de oscilação encontrados utilizando a modelagem com dois e três modos, na faixa de 0,2 a $0,4 \mathrm{~Hz}$. Observa-se que os valores de frequência e amortecimento encontrados utilizando a modelagem com dois modos são ligeiramente maiores, mas o comportamento dos valores é o mesmo para ambas as modelagens. 

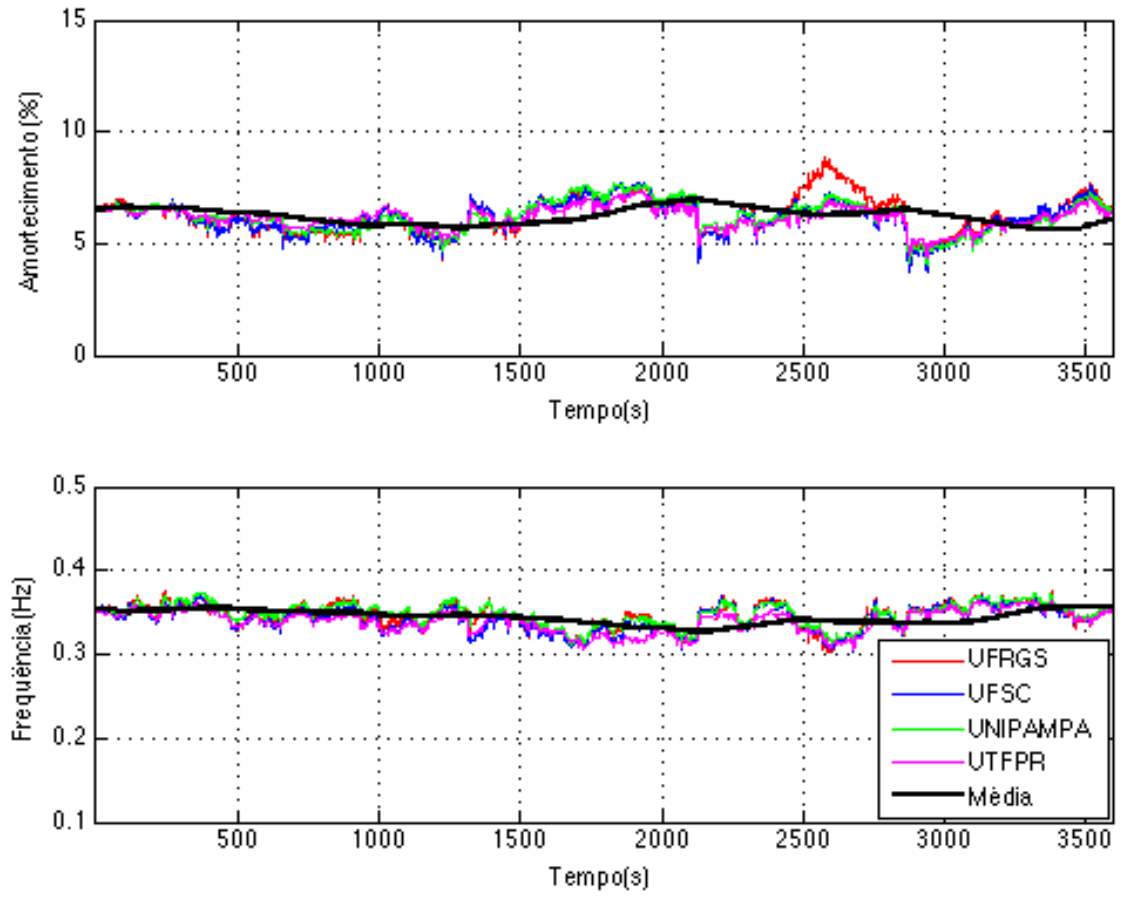

Figura 4.28: Média móvel de 10 minutos do amortecimento e da frequência do modo de oscilação identificado nos sinais de defasagem angular de tensão
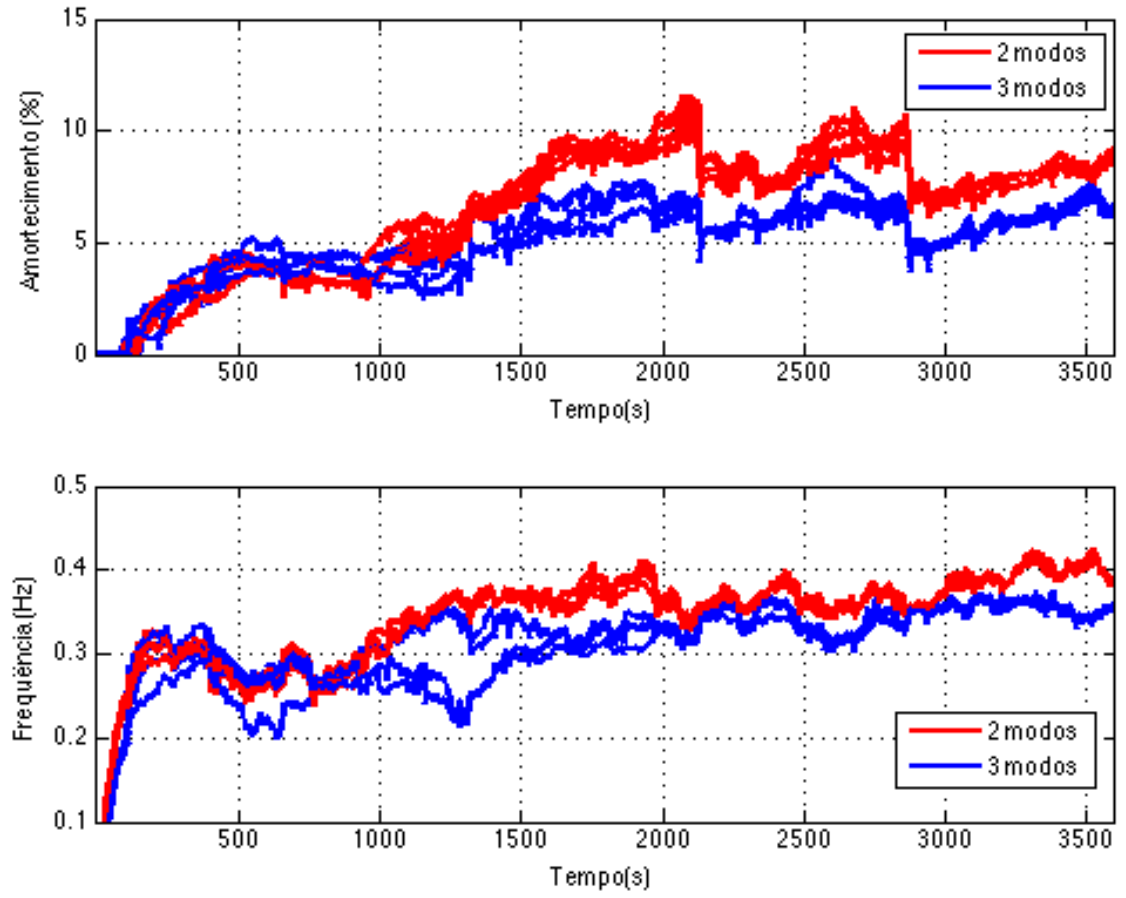

Figura 4.29: Comparação dos valores de amortecimento e frequência do modo de oscilação identificado utilizando modelagem com dois e três modos

\subsubsection{Sinais com referência na UFMA}

Nesta seção é apresentado o resultado de aplicação do algoritmo de FKE ao sinal de diferença angular de tensão da PMU instalada na UFC, na região Nordeste, tendo 
como referência o ângulo de tensão da fase A da PMU instalada na UFMA, no Norte.

A Figura 4.30 apresenta o espectro de frequências desse sinal após o pré-processamento descrito na Seção 3.3. Em vermelho está destacado o espectro do sinal antes da filtragem descrita na Seção 3.3.3, e em azul o espectro após essa filtragem.

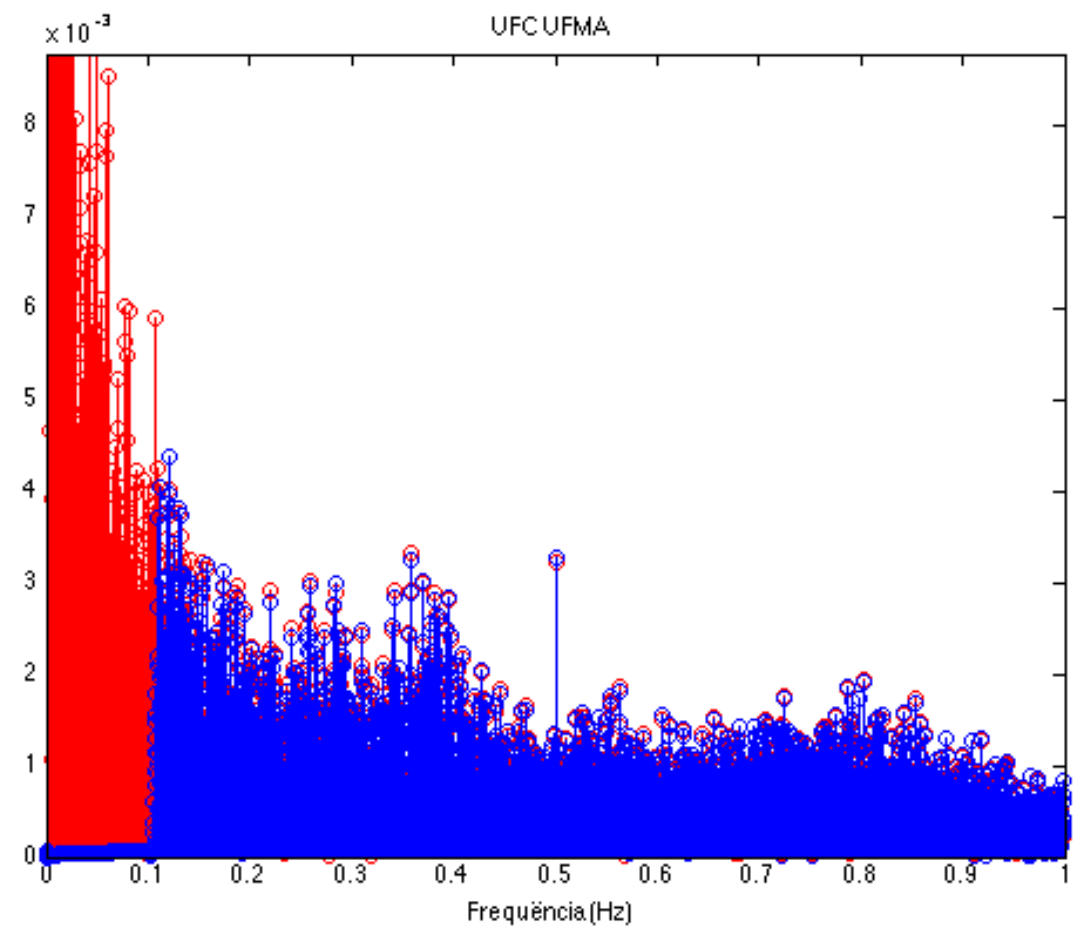

Figura 4.30: Espectro de frequências do sinal de defasagem angular de tensão entre a PMU da UFC e a da UFMA.

Neste caso, não é possível observar nenhum pico de magnitude no sinal na faixa entre 0,2 Hz e 0,4 Hz que indique a presença do modo de oscilação Norte-Sul. Esse resultado era de se esperar, tendo em vista que as duas PMUs em análise estão situadas nas regiões Norte e Nordeste. Não há observabilidade do modo Norte-Sul neste caso.

O algoritmo de FKE foi aplicado a este sinal utilizando a modelagem com três modos de oscilação. A Figura 4.31 apresenta os valores de amortecimento e frequência para os três polos calculados.

Observa-se que o algoritmo não converge para nenhum modo de oscilação com frequência e amortecimento característicos, tampouco para o modo de oscilação Norte-Sul. 

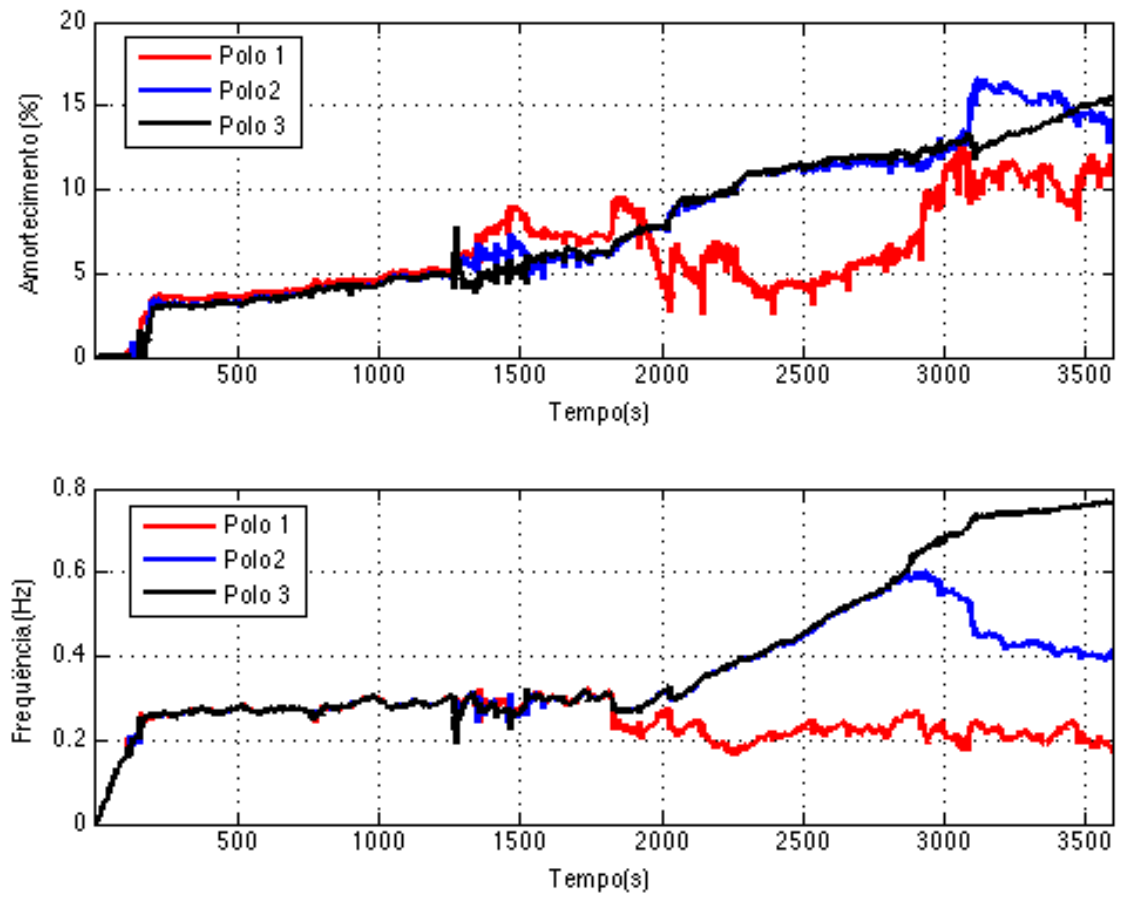

Figura 4.31: Amortecimento e frequência dos três polos calculados para o sinal de defasagem angular de tensão entre a PMU da UFC e a da UFMA, utilizando modelagem com três modos

\subsection{Conclusão}

Neste capítulo foram apresentados os resultados de aplicação do algoritmo de FKE para identificação de modos de oscilação dominantes em SEPs utilizando dados simulados no software PST bem como dados coletados em PMUs do SIN.

Nos testes com dados simulados o método apresentou rápida convergência dos valores de frequência e amortecimento identificados, apresentou valores coerentes com os valores reais e adaptou-se às variações dessas grandezas no terceiro caso simulado, em que o modo de oscilação dominante variava ao longo do tempo.

Os valores encontrados para frequência e amortecimento em todos os casos simulados foram ligeiramente diferentes dos valores reais dessas grandezas. A diferença entre os valores identificados e os valores reais para o amortecimento é maior do que para a frequência. Vários trabalhos relatam que os valores identificados frequentemente têm algum tipo de tendência, e que a obtenção de valores para o amortecimento é mais difícil do que para a frequência $[2,47,48,50,51]$.

O método baseado no espectro de frequências do sinal, adotado para escolha da quantidade de modos a serem utilizados na modelagem do sistema, mostrou-se simples de ser 
implementado e teve resultados satisfatórios. Muitos autores, inclusive, recomendam que os resultados encontrados utilizando métodos paramétricos sejam comparados com aqueles obtidos com métodos não-paramétricos como forma de verificação [2]. Nos casos dos sistemas simulados os modelos com apenas um ou dois modos foram suficientes para identificar o modo de oscilação dominante nos sistemas.

Finalmente, a aplicação do método proposto neste trabalho em dados de ambiente coletados de PMUs instaladas no SIN mostrou-se eficaz na identificação do modo de oscilação Norte-Sul do SIN, na faixa entre $0,2 \mathrm{~Hz}$ e 0,4 Hz. Foram analisados oito sinais de diferença angular de tensão nos quais foi possível observar a presença de um modo oscilação com frequência entre $0,35 \mathrm{~Hz}$ e $0,38 \mathrm{~Hz}$ e amortecimento entre $6 \%$ e $8 \%$. Estes resultados estão coerentes com os encontrados em outros trabalhos que buscaram identificar o modo de oscilação Norte-Sul do SIN [9, 61, 63]. 


\section{Conclusão}

\subsection{Conclusões gerais}

Esta dissertação propôs uma metodologia para estimação de modos de oscilação eletromecânica dominantes de um SEP a partir da análise de dados de ambiente obtidos a partir de medidores fasoriais. A metodologia utilizada para identificação dos modos foi formulada com base na teoria de FKE. A concepção do método é fundamentada na determinação de parâmetros que também são estados estimados do filtro.

O trabalho foi estruturado de modo que no Capítulo 2 foram apresentados os tipos de estabilidade de SEP com ênfase na estabilidade de ângulo do rotor associada a pequenas perturbações. Foram abordadas também as formas de avaliação da estabilidade por meio de modelos de sistemas elétricos e utilizando medições fasoriais sincronizadas. Por fim, foi apresentado levantamento bibliográfico sobre os principais trabalhos científicos que tratam da identificação de modos de oscilação e avaliação da estabilidade de SEPs a pequenas perturbações utilizando dados de ambiente coletados por meio de PMUs.

Já no Capítulo 3 destacaram-se informações básicas sobre PMU e sobre tratamento de dados visando identificação dos sinais. Dentre esses processos, enfatizou-se a importância de pré-processamento de dados, como o de interpolação. Neste contexto, destacou-se dados associados ao projeto Medfasee [10], base para os dados de campo utilizados nos testes. Neste capítulo também foram descritos e deduzidos os algoritmos do FK e do FKE, assim como a metodologia para a identificação de parâmetros na forma de variáveis de estado. Por fim, foi detalhada a modelagem em sistema de espaço de estados proposta nesta dissertação para identificação de modos de oscilação dominantes de SEP utilizando o FKE a partir de dados de ambiente e as formas de ajustes dos parâmetros iniciais do FKE.

Foram desenvolvidas no Capítulo 4 simulações de sistemas elétricos cujos modos de oscilação eram conhecidos, utilizando o software PST. Foram apresentados cinco casos simulados, com diferentes características dinâmicas, bem como os resultados obtidos da análise de sinais simulados de dados de ambiente utilizando a metodologia proposta nesta dissertação. Neste capítulo também foram apresentados os resultados da 
aplicação a sinais de defasagem angular de tensão coletados em PMUs instaladas no SIN.

Ficou demonstrado, através das simulações de modelos conhecidos de sistemas elétricos, que o método proposto estima os parâmetros do modo de oscilação dominante mesmo utilizando dados de ambiente, provenientes da operação normal do sistema. O método apresentou boa convergência nas simulações onde houve variação dos parâmetros do modo dominante, bem como mostrou-se adequado para avaliação contínua dos sinais, sem necessidade de reinicialização do processo ou de armazenamento de dados, tendo em vista tratar-se de um processo recursivo.

Os valores encontrados para frequência e amortecimento em todos os casos simulados estão de acordo com resultados práticos encontrados na literatura sobre o assunto, levando-se em conta a natureza estocástica do problema. Vários trabalhos relatam que os valores identificados frequentemente têm algum tipo de tendência, e que a obtenção de valores para o amortecimento é mais difícil do que para a frequência $[2,47,48,50,51]$.

A aplicação do método em dados de ambiente coletados de PMUs instaladas no SIN mostrou-se eficaz na identificação do modo de oscilação Norte-Sul. A identificação ocorre para a faixa entre $0,20 \mathrm{~Hz}$ e $0,40 \mathrm{~Hz}$, característica desse modo no SIN. Foram analisados oito sinais de diferença angular de tensão nos quais foi possível observar a presença de um modo oscilação com frequência entre $0,35 \mathrm{~Hz}$ e $0,38 \mathrm{~Hz}$ e amortecimento entre $6 \%$ e $8 \%$. Os resultados estão coerentes com os encontrados em outros trabalhos $[9,61,63]$.

O monitoramento do SIN utilizando dados de ambiente coletados por meio de PMUs mostrou ser uma importante fonte de informações a respeito do amortecimento do modo de oscilação Norte-Sul. E os resultados alcançados com a aplicação do método proposto foram satisfatórios para a análise da estabilidade de SEPs.

\subsection{Sugestões para trabalhos futuros}

Em trabalhos futuros, sugere-se que seja investigado o cálculo de modos dominantes em SEPs utilizando simultaneamente vários sinais coletados por PMUs, com o intuito de identificar as regiões de origem das oscilações, além dos parâmetros do modo. Além disso, alguns ponto merecem investigação específica, tais como: 
- Formas alternativas de interpolação de dados de PMU corrompidos que tenham resultados satisfatórios;

- Aprimoramento da identificação dos parâmetros de amortecimento e frequência dos modos de oscilação com o intuito de atenuar as tendências verificadas nos valores encontrados neste trabalho;

- Investigação de novas formas de estimação de parâmetros de sistemas não lineares, tais como o Unscented Kalman Filter e o FKE com equações de restrições [82]. 


\section{REFERÊNCIAS BIBLIOGRÁFICAS}

[1] Prabha Kundur, John Paserba, Venkat Ajjarapu, Göran Andersson, Anjan Bose, Claudio Canizares, Nikos Hatziargyriou, David Hill, Alex Stankovic, Carson Taylor, Thierry Van Cutsem, and Vijay Vittal. Definition and classification of power system stability. IEEE Transactions on Power Systems, 19(2):1387-1401, 2004.

[2] IEEE Task Force on Identification of Electromechanical Modes in Power Systems. Technical Report TP462, IEEE Power \& Energy Society, Junho 2012.

[3] Jimmy Chih-Hsien Peng, Nirmal-Kumar C. Nair, Jian Zhang, and Akshya Kumar Swain. Detection of lightly damped inter-area power oscillations using extended complex kalman filter. IEEE Region 10 Conference TENCON, pages 1-5, 2009.

[4] Prabha Kundur. Power System Stability and Control. McGraw-Hill Education, 1994.

[5] Luigi Vanfretti, Luke Dosiek, John W. Pierre, Daniel Trudnowski, Joe H. Chow, Rodrigo García-Valle, and Usman Aliyu. Application of ambient analysis techniques for the estimation of electromechanical oscillations from measured PMU data in four different power systems. European Transactions on Power Systems, 21(4):1640-1656, 2011.

[6] M. J. Gibbard, P. Pourbeik, and D. J. Vowles. Small-signal stability, control and dynamic performance of power systems. University of Adelaide Press, 2015.

[7] I. C. Decker, D. Dotta, Zimath S. L. Agostini, M. N., and A. S. Silva. Performance of a synchronized phasor measurements system in the brazilian power system. IEEE Power Systems Conference and Exposition, pages 1-8, 2006.

[8] Fernando B. Prioste, Aguinaldo S. Silva, and Ildemar C. Decker. Avaliação de métodos de identificação de modos eletromecânicos em sistemas elétricos de potência. XVIII Congresso Brasileiro de Automática, pages 3391-3398, 2010. 
[9] Thiago Jeremias, Valmor Zimmer, Ildemar C. Decker, Aguinaldo S. Silva, and Marcelo N. Agostini. Estudo de oscilações eletromecânicas no sistema elétrico brasileiro utilizando medidas fasoriais sincronizadas. XIX Congresso Brasileiro de Automática, pages 2364-2371, 2012.

[10] Projeto Medfasee da Universidade Federal de Santa Catarina - UFSC. Disponível: http://www.medfasee.ufsc.br/temporeal/.

[11] J. W. Pierre, D. J. Trudnowski, and M. K. Donnelly. Initial results in electromechanical mode identification from ambient data. IEEE Transactions on Power Systems, 12(3):1245-1251, 1997.

[12] D. J. Trudnowski. Order reduction of large-scale linear oscillatory system models. IEEE Transactions on Power Systems, 9(1):451-458, 1994.

[13] Francisco Damasceno Freitas, Joost Rommes, and Nelson Martins. Gramian-based reduction method applied to large sparse power system descriptor models. IEEE Transactions on Power Systems, 23(3):1258-1270, 2008.

[14] G. Troullinous, J. Dorsey, H. Wong, and J. Myers. Reducing the order of very large power system models. IEEE Transactions on Power Systems, 3(1):127-133, 1988.

[15] Juan J. Sanchez-Gasca and Joe H. Chow. Power system reduction to simplify the design of damping controllers for interarea oscillations. IEEE Transactions on Power Systems, 11(3):1342-1349, 1996.

[16] H. Y. Altalib and P. C. Krause. Dynamic equivalents by combination of reduced order models of system components. IEEE Transactions on Power Apparatus and Systems, 95(5):1535-1544, 1976.

[17] Lei Wang, Meir Klein, Solomon Yirga, and Prabha Kundur. Dynamic reduction of large power systems for stability studies. IEEE Transactions on Power Systems, 12(2):889-895, 1997.

[18] Z. Gallehdari, M. Karrari, and O. P. Malik. Model order reduction using PSO algorithm and it's application to power systems. International Conference on Electric Power and Energy Conversion Systems, pages 1-5, 2009.

[19] Joost Rommes, Nelson Martins, and Francisco Damasceno Freitas. Computing rightmost eigenvalues for small-signal stability assessment of large-scale power systems. IEEE Transactions on Power Systems, 25(2):929-938, 2010. 
[20] D. J. Trudnowski and J. W. Pierre. Overview of algorithms for estimating swing modes from measured responses. IEEE Power 83 Energy Society General Meeting, pages $1-8,2009$.

[21] Takuhei Hashiguchi, Masamichi Yoshimoto, Yasunori Mitani, Osamu Saeki, Kiichiro Tsuji, Masahide Hojo, and Hiroyuki Ukai. Analysis of power system dynamics based on multiple synchronized phasor measurements. IEEE Power Engineering Society General Meeting, 2:615-620, 2003.

[22] Juan J. Sanchez-Gasca and Joe H. Chow. Performance comparison of three identification methods for the analysis of electromechanical oscillations. IEEE Transactions on Power Systems, 14(3):995-1002, 1999.

[23] Jukka Turunen, Jegatheeswaran Thambirajah, Mats Larsson, Bikash C. Pal, Nina F. Thornhill, Liisa C. Haarla, William W. Hung, Alex M. Carter, and Tuomas Rauhala. Comparison of three electromechanical oscillation damping estimation methods. IEEE Transactions on Power Systems, 26(4):2398-2407, 2011.

[24] Jun-Zhe Yang, Chih-Wen Liu, and Wen-Giang Wu. A hybrid method for the estimation of power system low-frequency oscillation parameters. IEEE Transactions on Power Systems, 22(4):2115-2123, 2007.

[25] Ning Zhou, John W. Pierre, and John F. Hauer. Initial results in power system identification from injected probing signals using a subspace method. IEEE Transactions on Power Systems, 21(3):1296-1302, 2006.

[26] J. C.-H. Peng and Nirmal-Kumar C. Nair. Comparative assessment of kalman filter and prony methods for power system oscillation monitoring. IEEE Power $\mathcal{G}$ Energy Society General Meeting, pages 1-8, 2009.

[27] Jimmy Chih-Hsien Peng and Nirmal-Kumar C. Nair. Enhancing kalman filter for tracking ringdown electromechanical oscillations. IEEE Transactions on Power Systems, 27(2):1042-1050, 2012.

[28] Robert Chang and Tapan Kumar Saha. Improved extended complex kalman filter for identifying inter-area oscillations. IEEE International Conference on Power System Technology, pages 1-6, 2012.

[29] Mehrdad Yazdanian, Ali Mehrizi-Sani, and Mohsen Mojiri. A novel approach for ringdown detection using extended kalman filter. 39th Annual Conference of the IEEE Industrial Electronics Society, pages 3270-3274, 2013. 
[30] P. M. Anderson and A. A. Fouad. Power System Control and Stability. IEEE Press, 2003.

[31] L. L. Grigsby. The Electric Power Engeneering Handbook. CRC Press LLC, 2001.

[32] Luigi Vanfretti. Estimation of electromechanical modes in power systems using synchronized phasor measurements and applications for control of inter-area oscillations, $2013 . \quad$ http://www.divaportal.org/smash/get/diva2:638863/FULLTEXT01.pdf.

[33] W. D. Stevenson. Elementos de Análise de Sistemas de Potência. McGraw-Hill, 1986.

[34] Daniel J. Trudnowski, John W. Pierre, Ning Zhou, John F. Hauer, and Manu Parashar. Performance of three mode-meter block-processing algorithms for automated dynamic stability assessment. IEEE Transactions on Power Systems, 23(2):680-690, 2008.

[35] J. F. Hauer, D. J. Trudnowski, and J. G. DeSteese. A perspective on WAMS analysis tools for tracking of oscillatory dynamics. IEEE Power Engineering Society General Meeting, pages 1-10, 2007.

[36] I. C. Decker, D. Dotta, M. N. Agostini, A. S. Silva, B. T. Meyer, and S. L. Zimath. Installation and monitoring experiences of the first synchronized measurement system in the brazilian national grid. IEEE/PES Transmission and Distribution Conference and Exposition: Latin America, pages 1-7, 2008.

[37] P. Korba and K. Uhlen. Wide-area monitoring of electromechanical oscillations in the nordic power system: practical experience. IET Generation, Transmission $\mathcal{G}_{3}$ Distribution, 4(10):1116-1126, 2010.

[38] Aaron F. Snyder, Nouredine Hadjsaid, Didier Georges, Lamine Mili, Arun G. Phadke, Olivier Faucon, and Sylvain Vitet. Inter-area oscillation damping with power system stabilizers and synchronized phasor measurements. Proceedings on International Conference on Power System Technology, 2:790-794, 1998.

[39] Mohamed Ramadan Younis and Reza Iravani. Wide-area damping control for inter-area oscillations: A comprehensive review. IEEE Electrical Power $\&$ Energy Conference, pages 1-6, 2013.

[40] D. J. Trudnowski, M. K. Donnelly, and J. F. Hauer. A procedure for oscillatory parameter identification. IEEE Transactions on Power Systems, 9(4):2049-2055, 1994. 
[41] Jinyu Xiao, Xiaorong Xie, Yingduo Han, and Jingtao Wu. Dynamic tracking of low-frequency oscillations with improved prony method in wide-area measurement system. IEEE Power Engineering Society General Meeting, 1:1104-1109, 2003.

[42] P. Tripathy, S. C. Srivastava, and S. N. Singh. An improved prony method for identifying low frequency oscillations using synchro-phasor measurements. International Conference on Power Systems, pages 1-5, 2009.

[43] Ning Zhou, Zhenyu Huang, Francis Tuffner, John Pierre, and Shuangshuang Jin. Automatic implementation of prony analysis for electromechanical mode identification from phasor measurements. IEEE Power and Energy Society General Meeting, pages 1-8, 2010.

[44] Gerard Ledwich and Edward Palmer. Modal estimates from normal operation of power systems. IEEE Power Engineering Society Winter Meeting, 2:1527-1531, 2000.

[45] Jukka Turunen, Mats Larsson, Jerry Thambirajah, Liisa C. Haarla, and Tuomas Rauhala. Recent developments in modal estimation of power system electromechanical oscillations. IEEE Trondheim PowerTech, pages 1-7, 2011.

[46] Richard W. Wies, John W. Pierre, and Daniel J. Trudnowski. Use of ARMA block processing for estimating stationary low-frequency electromechanical modes of power systems. IEEE Transactions on Power Systems, 18(1):167-173, 2003.

[47] R. W. Wies and J. W. Pierre. Use of least mean squares (LMS) adaptive filtering technique for estimating low-frequency electromechanical modes in power systems. Proceedings of the American Control Conference, 6:4867-4873, 2002.

[48] Richard W. Wies, Ashok Balasubramanian, and John W. Pierre. Combining least mean squares adaptive filter and auto-regressive block processing techniques for estimating the low-frequency electromechanical modes in power systems. Proceedings of IEEE Power Engineering Society General Meeting, 2006.

[49] R. W. Wies, A. Balasubramanian, and J. W. Pierre. Adaptive filtering techniques for estimating electromechanical modes in power systems. IEEE Power Engineering Society General Meeting, pages 1-8, 2007.

[50] Ning Zhou, John W. Pierre, Daniel J. Trudnowski, and Ross T. Guttromson. Robust RLS methods for online estimation of power system electromechanical modes. IEEE Transactions on Power Systems, 22(3):1240-1249, 2007. 
[51] Ning Zhou, Daniel J. Trudnowski, John W. Pierre, and William A. Mittelstadt. Electromechanical mode online estimation using regularized robust RLS methods. IEEE Transactions on Power Systems, 23(4):1670-1680, 2008.

[52] I. Moreno and A. R. Messina. Adaptive tracking of ambient system oscillations by nonstationary RLS techniques. IEEE Power and Energy Society General Meeting, pages 1-8, 2011.

[53] P. Korba, M. Larsson, and C. Rehtanz. Detection of oscillations in power systems using kalman filtering techniques. Proceedings of IEEE Conference on Control Applications, 1:183-188, 2003.

[54] P. Korba. Real-time monitoring of electromechanical oscillations in power systems: first findings. IET Generation, Transmission \& Distribution, 1(1):80-88, 2007.

[55] Jukka Turunen, Liisa Haarla, and Tuomas Rauhala. Performance of wavelet-based damping estimation method under ambient conditions of the power system. VIII 2010 iREP Symposium Bulk Power System Dynamics and Control, pages 1-9, 2010.

[56] Jukka Turunen, Tuomas Rauhala, and Liisa Haarla. Selecting wavelets for damping estimation of ambient-excited electromechanical oscillations. IEEE Power and Energy Society General Meeting, pages 1-8, 2010.

[57] Jegatheeswaran Thambirajah, Nina F. Thornhill, and Bikash C. Pal. A multivariate approach towards interarea oscillation damping estimation under ambient conditions via independent component analysis and random decrement. IEEE Transactions on Power Systems, 26(1):315-322, 2011.

[58] Luke Dosiek, John W. Pierre, and Jim Follum. A recursive maximum likelihood estimator for the online estimation of electromechanical modes with error bounds. IEEE Transactions on Power Systems, 28(1):441-451, 2013.

[59] Mats Larsson and Dina Shona Laila. Monitoring of inter-area oscillations under ambient conditions using subspace identification. IEEE Power \&6 Energy Society General Meeting, pages 1-6, 2009.

[60] Ni Jingmin, Shen Chen, and Liu Feng. Estimating the electromechanical oscillation characteristics of power system based on measured ambient data utilizing stochastic subspace method. IEEE Power and Energy Society General Meeting, pages $1-7,2011$. 
[61] Fernando B. Prioste, Aguinaldo S. Silva, and Ildemar C. Decker. Monitoring oscillations modes of the brazilian interconnected power system using ambient data. IEEE Trondheim PowerTech, pages 1-7, 2011.

[62] S. A. Nezam Sarmadi and Vaithianathan Venkatasubramanian. Electromechanical mode estimation using recursive adaptive stochastic subspace identification. IEEE Transactions on Power Systems, 29(1):349-358, 2014.

[63] R. B. Leandro, A. S. Silva, I. C. Decker, and M. N. Agostini. Identification of the oscillation modes of a large power system using ambient data. Journal of Control, Automation and Electrical Systems, 26(4):441-453, 2015.

[64] Aguinaldo S. Silva. The Use of Synchrophasors in Power Systems, 2012. Disponível: http://www.eps.ee.kth.se/personal/vanfretti/events/brazilworkshop2012/pres/medfase.pdf.

[65] Richard Andrew Wiltshire, Gerard Ledwich, and Peter O'Shea. A kalman filtering approach to rapidly detecting modal changes in power systems. IEEE Transactions on Power Systems, 22(4):1698-1706, 2007.

[66] Luigi Vanfretti, Sebastian Bengtsson, and Jan O. Gjerde. Preprocessing synchronized phasor measurement data for spectral analysis of electromechanical oscillations in the nordic grid. International Transactions on Electrical Energy Systems, 25(2):348-358, 2015.

[67] F. N. Fritsch and R. E. Carlson. Monotone piecewise cubic interpolation. SIAM Journal on Numerical Analysis, 17(2):238-246, 1980.

[68] Arthur Gelb. Applied Optimal Estimation. MIT Press, 1974.

[69] M. S. Grewal and A. P. Andrews. Kalman Filtering: Theory and Practice using Matlab. John Wiley \& Sons, Inc., 2008.

[70] Rudolph Emil Kalman. A new approach to linear filtering and prediction problems. Transactions of the ASME - Journal of Basic Engineering, 82(Series D):35-45, 1960.

[71] P. S. Maybeck. Stochastic Models, Estimation and Control, volume 1. Academic Press, 1979.

[72] Greg Welch and Gary Bishop. An introduction to the kalman filter. Technical Report TR 95-041, University of North Carolina at 
Chapel Hill, Department of Computer Science, 2006. Disponível : https://www.cs.unc.edu/ welch/kalman/kalmanIntro.html.

[73] Simon O. Haykin. Adaptive Filter Theory. Prentice Hall, 1996.

[74] Erik Bolviken, Nils Christophersen, and Geir Storvik. Linear dynamical models, kalman filtering and statistics. lecture notes to IN-ST 259, 1998. http://www.mn.uio.no/math/tjenester/kunnskap/kompendier/komp_kalman.pdf.

[75] Vedran S. Perić and Luigi Vanfretti. Power system ambient-mode estimation considering spectral load properties. IEEE Transactions on Power Systems, 29(3):11331143, 2014.

[76] Katsuhiko Ogata. Engenharia de Controle Moderno. Ed. Pearson Education do Brasil, 2011.

[77] Emmanuel Blanchard, Adrian Sandu, and Corina Sandu. Parameter estimation method using an extended kalman filter. Proceedings of the Joint North America, Asia-Pacific ISTVS Conference and Annual Meeting of Japanese Society for Terramechanics, pages 23-26, 2007.

[78] K. J. Åström and P Eykhoff. System identification - a survey. Journal Automatica, 7:123-162, 1971.

[79] Joe H. Chow and Kwok W. Cheung. A toolbox for power system dynamics and control engineering education and research. IEEE Transactions on Power Systems, $7(4): 1559-1564,1992$.

[80] Graham Rogers, Joe H. Chow, and Luigi Vanfretti. Power System Toolbox Webpage. http://www.eps.ee.kth.se/personal/vanfretti/pst/Power_System_Toolbox_Webpage /PST.html.

[81] Boletins Diários da Operação - BDO do Operador Nacional do Sistema - ONS. Disponível: http://www.ons.org.br/resultados_operacao/boletim_diario/index.htm.

[82] D. Simon. Kalman filtering with state constraints: a survey of linear and nonlinear algorithms. IET Control Theory and Applications, 4(8):1303-1318, 2010. 